Illinois State University

ISU ReD: Research and eData

Theses and Dissertations

$9-5-2014$

\title{
Building Parent Support for Implementation of Assessment for Learning (A4L) at the High School Level
}

Sarah Highfill

Illinois State University, shighfill@lw210.org

Follow this and additional works at: https://ir.library.illinoisstate.edu/etd

Part of the Elementary and Middle and Secondary Education Administration Commons, and the Secondary Education and Teaching Commons

\section{Recommended Citation}

Highfill, Sarah, "Building Parent Support for Implementation of Assessment for Learning (A4L) at the High School Level" (2014). Theses and Dissertations. 250.

https://ir.library.illinoisstate.edu/etd/250

This Dissertation is brought to you for free and open access by ISU ReD: Research and eData. It has been accepted for inclusion in Theses and Dissertations by an authorized administrator of ISU ReD: Research and eData. For more information, please contact ISUReD@ilstu.edu. 


\title{
BUILDING PARENT SUPPORT FOR IMPLEMENTATION \\ OF ASSESSMENT FOR LEARNING (A4L) \\ AT THE HIGH SCHOOL LEVEL
}

\author{
Sarah K. Highfill
}

176 Pages

December 2014

Researchers articulate the positive effects of parental engagement with education. Networking with parents has become a recent focus for educational leaders working to enhance learning. With parent presence diminishing within secondary schools and emphasis on formative assessments rising, this study examined the possibility of family support to supplement the Assessment for Learning (A4L) reform implementation. Using an action research framework, four research questions were addressed. These included what attracts parents to support Assessment for Learning (A4L) reforms at the high school level, the value parents place on assessment reform, and what affects parent perceptions and influences their support of the assessment reform initiative. The fourth question inquired into how educational leaders can sustain home/school collaboration during the reform process. The three interlocking and overarching themes that emerged through analysis of the focus group data and surveys were Effort, Closing Gaps, and Fair Ranking. Subthemes for implementing A4L at the high school level highlight a process that (a) must encourage students' metacognitive and persistent effort as well as students taking responsibility for learning; (b) must encourage closing gaps identified in 
engagement, feedback, analysis, assessment, and culture; and (c) must incorporate discussions on fair ranking. The conclusion of the study offers recommendations for educational leaders desiring to make the collaboration between home and school a reality at the high school level. 


\section{BUILDING PARENT SUPPORT FOR IMPLEMENTATION \\ OF ASSESSMENT FOR LEARNING (A4L) \\ AT THE HIGH SCHOOL LEVEL}

SARAH K. HIGHFILL

A Dissertation Submitted in Partial

Fulfillment of the Requirements

for the Degree of

DOCTOR OF EDUCATION

Department of Educational Administration and Foundations

ILLINOIS STATE UNIVERSITY

2014 


\section{BUILDING PARENT SUPPORT FOR IMPLEMENTATION \\ OF ASSESSMENT FOR LEARNING (A4L) \\ AT THE HIGH SCHOOL LEVEL}

SARAH K. HIGHFILL

COMMITTEE MEMBERS:

Dr. Linda Lyman

Dr. Dianne Gardner Renn

Dr. Guy Banicki

Dr. Sharon K. Michalak 


\section{ACKNOWLEDGMENTS}

I would like to offer my sincere appreciation and gratitude to the many people who have shared their time and encouragement during my academic journey.

Thank you to Dr. Linda Lyman, chair of my committee, whose vision for improving the communication with the community is energizing. I would also like to thank my other ISU committee members, Dr. Dianne Gardner Renn and Dr. Guy Banicki, for their suggestions and contributions to this final project. I must also thank the friends that I made as a member of the Joliet cohort.

Thank you to the Lincoln-Way administration, especially Dr. Sharon Michalak. As my other committee member, you inspired my love for curriculum and showed how to balance both an administrative job and motherhood. Other administrators who believed in me before I even knew what I was capable of are: Tim Reilly, Lynn Merrick, and the late Dr. Kelly Ludwig. The most influential group at Lincoln-Way is the Science Department, whose dedication to student academic success makes me proud to be a part of and lead.

Thank you to my many friends who gently asked when this journey would end. Your encouragement and 'you can do it' attitude spurred me on. I especially would like to thank Jon Speck for his time and effort reviewing my grammar and sentence structure in my early writing that became the backbone of this document. 
Thank you to my family who displayed love and support each step of the way. To my parents, Steven and Deborah Knezovich, and to my in-laws, Thomas and Geraldine Highfill; I always knew I could lean on you when needed.

Thank you to my children Ethan, Noah, Grace, and Paul for your patience and understanding when mommy had to write. I hope my journey will inspire you to value your education today, tomorrow, and always. You can be whatever you set your mind to by showing perseverance, setting goals, and celebrating in victories.

And finally, thank you to my husband, Matthew. You have been most supportive, loving, and patient. Without you, this journey would have never begun nor ever completed.

S. K. H.

Success is about dedication. You may not be where you want to be, or do what you want to do when you're on the journey. But you've got to be willing to have vision and foresight that leads you to an incredible end. -Usher 


\section{CONTENTS}

Page

ACKNOWLEDGMENTS CONTENTS

TABLES

FIGURES

CHAPTER

I. INTRODUCTION 1

Statement of the Problem $\quad 2$

Purpose of the Study 4

Research Questions $\quad 5$

\begin{tabular}{ll} 
Definitions & 6 \\
\hline
\end{tabular}

Context of the Problem $\quad 8$

Action Research Design 11

$\begin{array}{ll}\text { Theoretical Perspective } & 12\end{array}$

$\begin{array}{ll}\text { Positionality } & 15\end{array}$

Delimitations and Limitations $\quad 17$

$\begin{array}{ll}\text { Significance of the Study } & 19\end{array}$

II. REVIEW OF LITERATURE 21

$\begin{array}{ll}\text { Leadership of Change } & 23\end{array}$

Challenges of Organizational Change $\quad 24$

Four frames categorization of organizational theory 25

Issues in managing effective change 26

$\begin{array}{ll}\text { Comparing Change Models } & 27\end{array}$

Kotter and Cohen (2002) model 28

Hall and Hord (2010) model 28

Viewing Change Models through the Bolman and Deal Frames 29 
Leading Through Change

Leadership approaches to involve parents in assessment reform

Assessment Practices Past and Present

Learning Standards as Reform 38

Incorporating Assessment into Reform

Comparing Achievement of Learning and Assessment for Learning

Issue one

Issue two

Issue three

41

Targeting active student learning

Challenges of Assessment Reform

Cultural adaptation

Grading crisis

Local Implementation of Assessment for Learning $\quad 49$

Parent/Family Engagement $\quad 52$

Transition from Involvement to Engagement 55

Defining involvement and engagement 55

Identifying policy and framework $\quad 57$

Supporting Academics and Learning at Home $\quad 59$

Factoring parental influence $\quad 60$

Addressing the Assessment for Learning Paradigm with Parents 64

Recommendations from past studies $\quad 65$

$\begin{array}{ll}\text { Chapter Summary } & 68\end{array}$

$\begin{array}{ll}\text { II. METHODOLOGY } & 70\end{array}$

Characteristics of Action Research $\quad 71$

The Living Educational Theory $\quad 74$

Research Purpose and Questions $\quad 76$ 
$\begin{array}{ll}\text { Data Collection and Procedures } & 78\end{array}$

$\begin{array}{ll}\text { Setting and Participants } & 79\end{array}$

Informal Presentation $\quad 80$

$\begin{array}{ll}\text { Surveys } & 81\end{array}$

2010 Asset program survey 81

$\begin{array}{ll}\text { Informal presentation survey } & 82\end{array}$

$\begin{array}{ll}\text { The 5Essentials survey } & 82\end{array}$

$\begin{array}{ll}\text { Focus group survey } & 83\end{array}$

Focus Group Interview $\quad 83$

Document Analysis $\quad 84$

Study Timeline $\quad 85$

Data Analysis and Procedure $\quad 86$

Quality of the Study $\quad 89$

$\begin{array}{ll}\text { Ethical Considerations } & 90\end{array}$

$\begin{array}{lr}\text { Chapter Summary } & 91\end{array}$

IV. FINDINGS AND DISCUSSION 93

Action Research Purpose $\quad 94$

Research Questions $\quad 95$

Action Research Findings 96

$\begin{array}{ll}\text { Research Question One } & 98\end{array}$

Metacognitive effort $\quad 99$

Closing the engagement gap 100

$\begin{array}{ll}\text { Research Question Two } & 101\end{array}$

Closing the assessment gap 102

Closing the feedback gap 104

Closing the analysis gap 105

Cultivating persistent effort 106

$\begin{array}{ll}\text { Research Question Three } & 109\end{array}$

Effort as responsibility 109

Fair ranking $\quad 110$

Closing the cultural gap $\quad 111$

Research Question Four 113

$\begin{array}{ll}\text { Chapter Summary } & 114\end{array}$ 
Implications

The Nature of Knowing

The Nature of Learning and Transfer

The Nature of Motivation and Engagement

Recommendations

Recommendations for Practice

Teachers

125

Administrators

Recommendations for Further Research

Conclusions

\section{REFERENCES}

APPENDIX A: Surveys

APPENDIX B: Focus Group Survey

APPENDIX C: Invitations and Consents

APPENDIX D: Self-Assessment Document 


\section{TABLES}

Table Page

1. The Differences Between Summative and Formative Assessment 42

2. Adopted Learning Strategies for District Alpha 50

3. The ACT's Top Twelve Assessment Verbs 102 


\section{FIGURES}

Figure $\quad$ Page

1. Comparing and Contrasting Leadership Approaches 34

2. Kemmis and McTaggart (1988) Model with Overlay of the CBM's SoC 87

3. The Study's Themes and Subthemes 97 


\section{CHAPTER I}

\section{INTRODUCTION}

We have work to do, but not impossible work. It is a matter of focus. It is a matter of appropriate criteria. It is a matter of communication. It is a matter of collaboration. It's a matter of will. (Hillard, 1997, p. 30)

Reform efforts over the last 20 years have attempted to appease skeptics by increasing graduation requirements, incorporating links between schools and career centers (Tech-Prep Education Act 1990 and the School-to-Work Opportunities Act 1994), redesigning high schools (block scheduling, smaller personalized schools, and school choice), and implementing standards-based learning. Standards-based learning is the reform effort that has had notable impact on today's educational leaders. Whether it is content standards - what students should know, or standards for proficient performance of skills - what students are able to do, the implications of standards for bringing about successful meaningful learning by high school students are overwhelming.

Students' graduation transcripts classify performance levels as below, meeting, or exceeding state standards. School districts are under public scrutiny because of low student performance. While this is understandably devastating to the district, students are largely unaffected by the scrutiny. Educational leaders must take ownership of standards-based assessment reform and evaluate progress toward its implementation. Such reform will benefit all students, if supported appropriately. Finding the equilibrium between standards-based learning and encouraging students to become more active in their learning is the work school districts need to do. 
It is a matter of focus on what is best for students long term. It is a matter of setting appropriate goals and finding ways to accomplish those goals. It is a matter of planning for sustainability, therefore a matter of collaboration. Encouraging studentinitiated learning within an environment focused on standards is a matter of will.

\section{Statement of the Problem}

Assessment reform vision must be enticing, explicit, and sweeping (Wiggins, 1998, p. 318).

Administrators in school districts attempting to initiate change in their buildings must be proactive, and they must create positive support. Stiggins (2007) stated, "I believe a vision of partnerships at this level of assessment holds immense promise" (p. 75). Stiggins' vision for collaboration to change schools includes the Assessment for Learning (A4L) concept. Assessments are known to parents as the method for checking in, comparing, and ranking student learning according to defined standards and/or to other districts and/or students (Guskey, 2006). However, if formative assessments are used to support learning and note the progress made toward reaching learning targets, then the traditional perception of assessment is challenged. The former concept is termed Assessment of Learning, while the latter addresses Assessment for Learning and hereafter will be identified through the acronym A4L. "The key to success is finding the synergy between the two concepts" (Stiggins, 2007, p. 70).

Black and Wiliam's (1998) research made assessment reform enticing by promoting the positive effects of formative assessment as learning, especially with its spotlight on involving more students in their own learning (Black \& Wiliam, 1998; Marzano, 2003; Stiggins, 2002, 2007; Wiggins, 1998). The vision of Assessment for 
Learning (A4L) encourages explicit classroom instruction by presenting identifiable goals for lessons, incorporating a focus on vocabulary, and stressing summarization skills. "If desired learning goals or standards are the foundation of student's instructional experiences, then assessments of student learning are simply extensions of those same goals and standards" (Guskey, 2007, p. 18).

In spite of this, formative assessment is not achieving sweeping adoption. Ten years after the concept's nationwide implementations, Boyle and Charles (2010) researched the application of the formative assessment framework in British primary schools. They unveiled that what was expected by the administration and what truly happened in classrooms did not correlate. Prior to their study, Carless (2005) and Cheng, Andrews, and Yu (2010) noted similar resistance to changes made in assessment practices in Hong Kong after 6 years of conducting research. In all studies, attempts at reforming assessment practices failed due to the lack of consensus on the nature of assessment and resistance to change. This was not surprising as Prestine and McGreal (1997) acknowledged the difficulty with staff buy-in of authentic assessment almost a decade before due to exhaustion and the lack of enthusiasm for trying new things. Carless (2005) adds that the reluctance of schools to accept assessment changes is a challenge. "All deep educational changes are challenging and assessment cultures seem to be particularly impervious to transformation" (p. 52). Validating this statement from more of an educational psychology perspective, Greeno, Collins, and Resnick (1996) proposed merging the cognitive themes of knowing, learning, and motivation to bridge theoretical and practical understanding of an educational practice. 
The desired transformation of Assessment for Learning (A4L) is students investing more time addressing their mistakes and correcting them through selfassessment methods. This change in student behavior demands support. As teachers and students work together in the classroom on the instructional goals, parents also share responsibility in supporting and nurturing the educational environment at home. Epstein (2011) asserts that educators who value parental involvement and actively seek to include parents create better school environments for students. Exposing parents to the educational initiatives and acquiring feedback may be the decisive steps needed to best implement the assessment reform.

The challenges of creating collaboration between home and school includes time constraints, established perceptions from both school and parents, and the decline of parental involvement during high school. Nonetheless, the bottom line is if parents care about their children's school success, they will want to be included in the educational process (Caplan, 2000). Administrators should reach out to parents to encourage and support the goals of formative assessment. The educational leader plays a vital role in defining, supporting, and instituting assessments' effectiveness (Reeves, 2007). Unfortunately, at this time, there are only a limited number of studies conducted from the family's point of view of assessment, and this deficiency creates a challenge for the educational leader.

\section{Purpose of the Study}

The broad purpose of this action research study was to identify how a high school district can initiate a partnership between home and school in order to support high school students through Assessment for Learning (A4L) changes. Constructs from the 
Stages of Concern (SoC) in the Concerns-Based Adoption Model (CBAM) developed by Hall and Hord (2010) were used to determine the progress of the study. The researcher examined the perceptions of a family partnership of A4L through parental information nights, surveys and self-assessment documents, and a focus group discussion.

Additionally, this study created a living educational theory (Whitehead, 2008) through action research that sought to build collaboration with parents to support implementation of Assessment for Learning (A4L) at a suburban high school district south of Chicago.

\section{Research Questions}

Since the 1980s, researchers have doted on the positive effects of parental involvement with education. However, with parental presence diminishing within secondary schools and emphasis on assessments rising, high schools must partner with parents to create a structure of support. The Assessment for Learning (A4L) initiative is designed to actively involve students in their learning by acknowledging learning goals and assuming responsibility to reach those goals. Students may initially struggle with assessment reform. Therefore, reaching out to parents/family for support, while perhaps creating the possibility of a cultural change, is a critical step to take. More specifically, this action research study sought to answer the following questions:

1. What attracts parents to supporting Assessment for Learning (A4L) reforms at the high school level?

2. How can parents value assessment reform and comprehend the goals of Assessment for Learning (A4L)?

3. What affects parent perceptions and influences their support of the district's Assessment for Learning (A4L) initiative? 
4. How can educational leaders sustain home/school collaboration during the Assessment for Learning (A4L) reform process?

\section{Definitions}

Assessment is a broad topic relevant to how we educate students and improve student performance (Wiggins, 1998, p. 7). Activities undertaken by teachers and their students in assessing themselves provide information to be used as feedback to modify both teaching and learning (Black \& Wiliam, 1998). Throughout this dissertation study, the following definitions will be assumed:

Assessment Practices: This term refers to the process of how teachers gather information from students, in order to assess how students learn and to provide feedback (Shepard, 2000; Hargreaves, 2005).

Assessment of Learning: This is a measurement of student achievement and a gauge of what students have learned (Stiggins, 2002), also regarded as summative assessment.

Summative Assessment: This term refers to assessment that is accompanied by a number or letter grade commonly associated with standardized tests (Stiggins, 2005b; McMillan, 2007; Paine, 2008) to pass judgment on the academic progress made by a student.

Formative Assessment: Assessment used to impact learning by measuring student progress in learning by identifying strengths and weaknesses in the student's knowledge during instruction (Stiggins, 2005b; McMillan, 2007; Paine, 2008). The planned process includes frequent cycles of formative feedback to also adjust instructional procedures (Popham, 2008). 
Assessment for Learning: This is a "process of seeking and interpreting evidence for use by learners and their teachers to decide where the learners are in their learning, where they need to go, and how best to get there" (Boyle \& Charles, 2010, p. 286), according to particular standards.

Self-Assessment: An evaluation of one's own abilities and failings (Chappuis, 2005). "Students reflect on and evaluate the quality of their work and their learning, judge the degree to which they reflect explicitly stated goals or criteria, and identify strengths and weaknesses in their work, and revise accordingly" (Andrade \& Du, 2007, p. 160).

Parent(s): Refers to not only natural parents but also legal guardians or other person(s) with whom a dependent student lives (Weaver, 2007) and who is considered the student's family.

Parent/Family Engagement: How involved the parent/family is with the child and the child's best interest, especially education. It is the partnership between school and the home designed to create support for educational initiatives (Ferlazzo \& Hammond, 2009).

Concerns-Based Adoption Model (CBAM): The model emphasizes that as people experience change, their comfort level depends upon the kinds of questions they ask and their use of whatever the change is (Hall \& Hord, 2010). The model includes seven stages of concerns ranging from awareness to refocusing.

Living Educational Theory: This is an individual's unique explanation for their educational influence of their own learning (Whitehead, 2008). The theory is sparked by questioning how can I improve what I am doing in order to generate knowledge. 


\section{Context of the Problem}

In order to identify how a high school district can invite families to become partners with the school, District Alpha was chosen as the subject of this research. District Alpha split as of 2009 into four self-sustaining schools serving 7,346 students, according to the 2013 school report card. Employing only five superintendents during 60 years, the district has held tight to the traditions and values of student achievement and fiscal responsibility as set by the Board of Education.

District Alpha's standards and assessment reform process began with a strong focus on curriculum and instruction grounded in research and data analysis. District Alpha has accurately anticipated stumbling blocks before they occurred and productively implemented solutions that served District Alpha's best interest. All professional development opportunities have linked curriculum and instruction together, but it is only recently that the push for more accountability has driven assessment into focus.

The district's first attempt at organized professional development was in 1998 with a consulting firm. Development opportunities were designed to expose teachers to classroom activities designed for particular learning styles rather than addressing actual learning standards. The consultants' approach at District Alpha was to improve students by improving instruction. This consisted of strategic teaching incorporating researchbased instructional strategies, adapting strategies to meet particular learning goals, and developing a school culture that supports teachers as they work together to master new strategies and refine their practice (Silver Strong \& Associates, 2011). Unfortunately, the shotgun approach of strategic teaching did not meet the criteria of Goals 2000, nor did it address how students learn or should be assessed. 
The next organized professional development at District Alpha was the Understanding by Design (UBD) unit planning model created by Wiggins and McTighe (Wiggins, 2001). The goal of working backwards by first identifying the desired results, then planning lessons accordingly, is the backbone of the UBD program. The question asked before embarking upon curriculum review is "how do we design work for students that develops deep, enduring understanding of key concepts and processes that will serve students well over the course of their lives?" (Wiggins, 2001, p. 1). This was the first time that assessment, not just teaching to a test, was a focus for District Alpha classrooms. This reform initiative still thrives at District Alpha in 2014. Each academic department uses the UBD template, including standards, essential questions, knowledge and skills, and assessment types for each unit taught.

To support assessment accountability, the district began to collect performance data on summative assessments via the Abacus program. Unfortunately, the program's ambiguous science standards were more like broad category labels such as 'forces', 'cells' or 'reactions'. Using the program became a labor intensive nightmare, only mandated by the district during final exams to fulfill NCLB data recommendations. Any feedback on assessment was quickly overlooked as the teaching staff did not have any accountability to, or belief in, the system. There was a need for change.

Dr. Bobb Darnell became District Alpha's next consultant in 2007, bringing the district its first taste of Assessment for Learning (A4L). As the district's consultant, Dr. Darnell brought the research studies of Black and Wiliam (1998), Wiggins (2001), Stiggins $(1999,2002)$ and Marzano $(2003,2007)$ to life, and made the district accountable for eight instructional strategies. These research-based strategies, influenced 
by the assessment for learning experts, provided professional development resources and opportunities for District Alpha. Baker, Bakken, Blum, Cates, Swerdlick, and Thompson (2007) would approve of District Alpha's abandonment of the annual 'next best thing' to remain true to one plan: the Assessment for Learning (A4L) program for the next several years. Most of the previous and present district improvement plans reflect professional development opportunities based on the following eight learning strategies:

1. State and show learning objectives at the beginning and end of each lesson.

2. Explicitly teach vocabulary as well as retention and memory strategies.

3. Encourage student self-assessment and adjustment.

4. Explicitly teach learning skills and strategies using summaries for patterning, thinking, and writing.

5. Provide frequent feedback to students about their learning related to the objective within 48 hours.

6. Explicitly teach learning skills and strategies using graphic organizers for patterning, thinking, and writing.

7. Provide corrective and enrichment activities that respond to student progress and provide additional opportunities that allow students to demonstrate learning.

8. Help student activate and build background information and advance organization.

The district started slowly and developed networks of support to get all staff members on board. This is one reason why, in 2009, the Board of Education changed the administrative role of Director of Data Analysis to the Director of Instruction. With the 
Director of Instruction taking over where Dr. Darnell left off, more staff development was created within District Alpha, and the district continues to work on Assessment for Learning (A4L). The Abacus data collection system morphed into Mastery Manager. This more user-friendly program is utilized for daily assessments, not just for final exams. The web-based features allow for easy access and evaluation of student performance based on the standards of each UBD topic.

Since 2001, the models of professional development now in use feature ongoing teacher development structures for both individual and collaborative teams. These exemplify sustainability that will only form from networks, connections, and when a considerable amount of effort is put forth. By adopting more continuous and ongoing support for training, the district sought out teacher leaders, and the change has been positive. With favored acceptance of change thus far, the district prefers the Assessment for Learning (A4L) initiative to work from the ground up. Administrators invite teachers to participate in the assessment for learning teams in order to conduct open discussions of the objectives of Assessment for Learning (A4L).

\section{Action Research Design}

Action research is the method most appropriate for research carried out within the researcher's own setting. With my role as both teacher and department chair in District Alpha, the daily interaction and involvement of implementing the high school district's goal of assessment for learning is a high priority. Action research is appropriate as intentional inquiry (Cochran-Smith \& Lytle, 1993, p. 5) to improve student achievement, to interact between two entities and to remain local and perform research in your own backyard (Creswell, 2009). Attempting to be an educator-researcher on a larger scale 
than just a classroom requires a review of literature and a study design to report how support between home and school can be established. This educational task of figuring out how parents can play a supportive role in Assessment for Learning (A4L) was very real and practical.

This action research also fills a local niche, since feedback from my 2011 pilot study requested further collaboration with parents. From a small interview sample, parents of three high school students (one freshman and two seniors) noted that much more time is needed to understand assessment in general, Assessment for Learning (A4L) specifically, and how they can support students through the assessment reform. This study examined the topic of assessment reform practices at the high school level focused on parent/family support through the following steps: parental information nights, surveys, and a focus group. The study:

- Introduced the concepts of Assessment for Learning (A4L).

- Reported upon study habits at home and in school.

- Uncovered the opinions and perceptions of parents regarding the objectives of the A4L.

- Offered a solution to how parents can become support for A4L.

- Adjusted reform strategies after the focus group to include teachers.

\section{Theoretical Perspectives}

Action research falls in the qualitative paradigm. The theoretical perspectives supporting the study are a form of action research called living educational theory (Whitehead, 2008), and the Concerns Based Adoption Model (CBAM) developed by Hall and Hord (2010). The implementation gap that I have noticed while 'walking the walk 
and talking the talk' of Assessment for Learning (A4L) is the lack of support. I designed a study that demonstrates leadership, creates partnerships, and develops future opportunities for parents to remain supporters of the academics in the high school. As explained by Whitehead (2008), a living educational theory emerges to best explain how teacher researchers are inspired to learn "how do I improve what I am doing?" linking to a goal of formative assessment. The living theory process utilizes action reflection cycles. "The creation of living theories begins in practice" (Whitehead, 1998, p. 2). Whitehead further elaborated that "living educational theories are created by action researchers' studies of singularities" (1998, p. 3). A summary from Whitehead (1989) best highlights motivation for development of the theoretical framework:

In a living approach to educational theory, action researchers present their claims to know how and why they are attempting to overcome practical educational problems in this form:

- I experience a problem when some of my educational values are negated in my practice

- I imagine a solution to my problem

- I act in the direction of my solution

- I evaluate the outcomes of my actions

- I modify my problems, ideas, and actions in the light of my evaluation. (p. 98)

Even though the district would like to move forward with Assessment for Learning (A4L), the district struggles with the implementation. The district requests the assessment reform to be bottom-up instead of top-down but does not provide a frame to accomplish the goal. Therefore, as an action researcher, I construct my own living educational theory to improve practice. I believe building support with parents to be a vital part of the support high school students can receive through assessment changes.

I do not like using the word 'I' when addressing research, but the living educational theory approach promotes using ' $I$ ' as it is the ' $I$ ' who is conducting the 
research. This theoretical perspective embraces the creation of a theory as an explanation of the researcher's professional learning. Whitehead (1989) reviews the definition of a theory as a common sense statement that may not be accepted as good or true, but simply is a logical approach. Therefore, I want to attempt to create such a theory: an original contribution to the educational field that challenges the current practice of Assessment for Learning (A4L) by introducing collaboration to strengthen the A4L goals.

Since enacting thoughts and concerns of parents was new within the district, the Concerns-Based Adoption Model (Hall \& Hord, 2010), specifically the Stages of Concern, was used to guide the methodology of action research as well address the culture change. Changing the status quo is a challenge, and this dissertation study addressed change using the Concerns-Based Adoption Model (CBAM). This change model is grounded in the belief that change is a personal process, and sometimes it is more important to attend to the personal aspects of change than it is to focus on the use of the innovation (Hall \& Hord, 2010, p. 68). Therefore, the CBAM model addresses the Stages of Concern (SoC). Using this model allowed this research to address the feelings, perceptions, and concerns regarding an innovation, thus making change a more manageable task. Change is addressed in simple concepts to more complex as the innovation becomes more second nature. The CBAM model defers to the scientific method and mirrors the steps of action research. All three approaches (CBAM, the scientific method, and action research) address change by recognizing a problem, observing, collecting data, creating and executing a plan, and continuously evaluating and evolving toward a better solution. 
The Kemmis and McTaggart (1988) model for action research uses planning, acting, observing, and reflecting to complement the Stages of Concern used in the Concerns-Based Adoption Model. As the SoC embraces change with awareness and information, the Kemmis and McTaggart model used for educational practices begins with a plan to improve those practices. Once a plan is solidified, the next phase of the Kemmis and McTaggart model is observing and acting toward managing change as it becomes more personal as suggested by SoCs. Finally, reflecting on the ongoing progress of change requires collaboration and ultimately refocusing to create a better plan.

\section{Positionality}

With my role in the education field, I agree with Cochran-Smith and Lytle (1993) that my determination to include families in the assessment reform derives from my knowledge of the professional practices related to teaching and learning. Hired for the 2000/2001 school year in the science department and remaining in the district since, I have been a part of the district's commitment to provide the experiences and opportunities necessary to maximize the academic and social growth of all students. I attended Understanding by Design (UBD) in June 2001. At that time, I was naïve to the impact that this program would have on the future of curriculum and instruction. Since professional development at that time was more a 'sit and get' stage (Model A from Gardner, Baker, Vogt, \& Hodel, 2005), I was informed about the educational trend, but I did not incorporate it. Science's curriculum review in 2004 only embraced the UBD's essential questions, instead of fully understanding the organization of UBD. 
It was not until 2007, when I was hired as the science department chair for District Alpha's Central campus, that my role as curriculum leader was apparent. With the UBD training, I led the district science department through its next curriculum review in 2010 incorporating the full UBD design. The conversion from Abacus to Mastery Manager was easier since the department was able to identify the learning standards used in instruction. My transition from full time teacher to both teacher and department chair in 2007 came at a perfect time. Dr. Darnell advocated the benefits of better assessment and engaging students in their learning; my interest peaked. Adding the heightened focus on Prairie State Achievement Exam (PSAE) scores and political implications, I had concerns regarding assessment reform while balancing the pressures of standardized tests.

I became active in Assessment for Learning (A4L) teams and used every opportunity to discuss assessment for learning in monthly department meetings. I knew the answer did not lie with the elementary district's approach. Directed by the administrative team at the junior highs, the elementary district's philosophical tenets regarding assessment as a grading practice were put in place at the beginning of the 2010/2011 school year without any input from teachers or parents. The goals of the grading practice were to provide specific guidelines on grading to promote consistent communication and foster positive attitudes/experiences about grading. The elementary district enacted assessment reform as a grading policy that was more teacher-oriented, while the high school has been building reform momentum with assessment strategies to engage students and develop skills for more responsible learning. The results of the assessment reform at the junior high level are weak support from teachers, and more 
importantly, misconceptions and unanswered questions by parents. This is why I was convinced that the high school district should learn from these junior high results and include parent involvement with changes in assessment.

During my 2011 pilot study, I reviewed the results from the district-wide student body survey. The survey revealed that high school students recognize that their parents are supportive of their academic endeavors in school. The specifics of how parents are supportive remain vague, and currently there is no district policy encouraging parent engagement. Another drive for completing this dissertation study is the elementary district that feeds into two of District Alpha's high schools. At the middle school, the elementary district began its assessment reform in the fall of 2010 without including parental engagement. The results of the middle school assessment reform were weakly supported by teachers, but more importantly presented misconceptions and unanswered questions from the parents. It is vital that District Alpha learns from these results and includes parent engagement with changes in assessment. Yet there are few instances when this suburban high school district has reached out for the opinions and concerns of the parents within the community. This action research must act as a catalyst to involve parents in their child's education. It is imperative that parents realize how important their involvement is to their child's success in school (Chadwick, 2004).

\section{Delimitations and Limitations}

A good study is conducted ethically, and the data says what you need it to say, thus displaying trustworthiness. Reliability in data reflects trustworthiness as it provides enough information for a reader to be able to make reasonable comparisons to other situations (MacLean \& Mohr, 1999), and how similar effort would work to summarize 
and be applicable to other situations (Cochran-Smith \& Lytle, 1993). This study is delimited, however, to only a single school district in the Midwest.

With any qualitative study, the subjective nature of the study can be viewed as a limitation for the study. The convenience sampling of the participants provided a limitation to the study. The study included the information or data received from volunteers. The study assumed parents would want to participate in the survey and focus group to become partners in assessment reform at the high school. It also assumed parents would want to interact with their teenagers, something about which this researcher lacks personal experience.

Associated with action research, another potential limitation is the location of the research being the district where the researcher is employed. I was the primary instrument for data collection and analysis (Merriam, 2009). This prominent role of the researcher in the study may pose a concern. It is possible that the parents may feel comfortable telling the researcher what is on their minds; however, they may respond with what they think the researcher wants to hear. On the other hand, I have experience with past qualitative studies that I have conducted, including a pilot study in the district. During the pilot study, I interviewed parents interested in Assessment for Learning (A4L) and their feedback provided an invaluable start to this research and gave this study direction. My relationship with the administrative team is another possible limitation; however, I requested support and their excitement for the study's conclusion has kept this research moving forward. 


\section{Significance of the Study}

Formative assessment is used to impact learning by measuring student progress in learning through identifying strengths and weaknesses in the student's knowledge. The main pillars of formative assessment are improving the accuracy of classroom assessments by addressing learning targets, providing feedback, and promoting activities of self-assessment (Black \& Wiliam, 1998; Chappuis, 2005; Stiggins, 2007; Wiggins, 1998). The groundbreaking meta-analysis by Black and Wiliam (1998) asserted that the proper implementation, training, and nurturing of formative assessment goals are "amongst the largest ever reported for educational intervention" (p. 61). Putting it into perspective, it is like an average scoring school showing advancements in summative assessments to become a top five school for its state. Even though A4L indicates success in student learning since 1998, it was not until recently, with the new evaluation system for administrators and teachers requiring a student data section, that districts were motivated to consider assessment reform and how best to implement reform strategies. By means of action research, this dissertation study addressed the start of assessment reform in a Midwestern suburb.

Promoting a change in assessment is a change that must be valued by teachers, students, and the community (Shepard, 2000). Since families have a limited understanding of assessment beyond the meaning of grades, it is crucial that school districts should continue to enhance parents' capacity of understanding the current state of high school curriculum under standards implementation (Hoover-Dempsey, Walker, Whetsel, Wilkins, \& Closson, 2005) and how the curriculum approaches student learning. The key is the collaboration with parents and authentically listening to them. Auerbach 
(2009) addresses the need for parents to become and remain involved in their students' academic careers. This dissertation study was the opportunity for administrative leaders to promote meaningful engagement that will link back to student success. Auerbach (2007) found that administrators believe parent involvement is a tool for raising student achievement; therefore, to create opportunities to discuss the value and impression of assessment and its current significance in standards-based learning will be innovative. Webb and Jones (2009) wondered how whole school development of formative assessment is possible. It is significant to state that the Assessment for Learning (A4L) practice will present difficulties, since the initiative will push back on the traditional perception of assessment, but the power of change should not be underestimated. Prestine and McGreal (1997) suggested that school restructuring initiatives should start small with the classrooms and build to districts. "Changes work best when they are decided on by the level responsible for implementation" (p. 397). If District Alpha places high expectations for more student-initiated learning within its classes, then working with parents is a valid option. Webb and Jones (2009) note that "expectations are important in enabling successful classroom practice” (p. 173). Schmoker (2004) would agree as he notes, "Once the infrastructure for improvements includes common standards and assessment, the opportunity for effective leadership emerges" (p. 5). The dissertation study built upon parent/family opinions and perspectives of Assessment for Learning (A4L) to ultimately partner with them to establish a support system for student learning. 


\section{CHAPTER II}

\section{REVIEW OF LITERATURE}

A Nation at Risk has been known as the springboard into assessment accountability, with its harsh opinion that America was the only one to blame for its "mediocre educational performance" (National Commission of Excellence in Education, 1983, p. 5). Suggested solutions to mediocrity were to increase graduation requirements, push problem solving activities, address higher standards taught in the classrooms, and close the gap on assessment. In the early 1990s, assessment tried to reflect what students knew, while at the same time determine accountability for learning for school districts and teachers. The phrase 'teaching to the test' emerged. Assessment practices remain scrutinized, and seek ways to be formative (Stiggins, 2005b). Currently, there has been no attempt to push a nationwide assessment reform in the United States, even though mild success has been documented in both England (Boyle \& Charles, 2010) and Hong Kong (Carless, 2005; Cheng, Andrews, \& Yu, 2010). The value of assessments remains local within each state and within each school district. With emerging changes in assessment, this dissertation research explored opportunities for educational leaders to become partners with parents to help transition to new assessment practices within their high schools.

Networking with parents has become a recent focus for educational leaders. Chadwick (2004) acknowledges if the community is engaged with the school district, 
more improved teaching and learning follows. Therefore, it is vital that engagement initiatives work at a shared vision regarding the purpose of public school education, one that includes mastery of skills, intellectual development, and self-realization (Goodlad, 1994). Parents are essential to any educational reform effort. Shepard and Bliem (1995) highlight that parents “support their children's learning and collectively they can unseat professionally developed, research based curriculum and assessment changes" (p. 30). Agreeing with Shepard and Bliem, Yamamoto and Holloway (2010) state it is worth the risk to involve parents and to "understand the dynamic process of family members interacting with each other and with others in the school and community" (p. 208). If an administrator is willing to listen to parent perceptions of assessment practice, it will strengthen the assessment initiatives at the high school, cultivate a desire to learn from its students, and find support within the community for curriculum and instruction.

The opportunity to change the direction of assessment is real, current, and ready to be implemented. However, the obstacles include that different stakeholders will set diverse priorities for an assessment system (ETS, Pearson, \& College Board, 2010), as well as the prevailing belief that assessment is entirely about measurement (Chappuis, 2007). As long as our educational system only uses assessment as a means to rank schools and students, it is possible that education will miss assessments' most powerful benefits (Guskey, 2003)—i.e., to promote life-long learning.

There are three areas of focus within the review of literature. The first section of the chapter will focus on change, presenting a review of change as an organizational challenge, change models, and leadership for change. The organizational challenge is to make active student learning a reality in District Alpha high schools. To Senge (2000), 
Bridges (2003), Hall and Hord (2010), change and its transitions are manageable and welcomed as change makes advancements toward student achievement workable. Demonstrating particular leadership styles can influence school administrators to best manage changes in both assessment and parent engagement. The second section of the chapter will overview assessment practices past and present, as well as identify and discuss challenges of assessment reform. The process is already underway. With the district's focus and commitment to eight learning strategies, the next step for sustainability of assessment reform is parent engagement. Standards-based learning is the educational reform most relevant to the application of Assessment for Learning (A4L), the focus of this research. Finally, the chapter will present research about parent engagement, about how to move from parent involvement to engagement, including processes for involving parents as collaborative partners in assessment reform with the school district. Parent involvement does diminish at the high school level, but it is not extinct. This dissertation research was an opportunity to identify and understand parents as resources for school administrators during assessment changes.

\section{Leadership of Change}

All deep educational changes are challenging and assessment cultures seem to be particularly impervious to transformation. (Carless, 2005, p. 52)

The Assessment for Learning (A4L) initiative will challenge the traditional perception of assessment. The promise of formative assessment should rest with school leadership teams (Black, Harrison, Lee, Marshall, \& Wiliam, 2004); however, the fulfillment of that promise will take collaboration and support from everyone involved, including parents. In the following sections, the review of literature will discuss the 
principles of organizational theory in framing the challenges of assessment reform. With change imminent in District Alpha, two different change models will be compared. Finally, the leadership style promoting the most successful assessment reform will be suggested.

\section{Challenges of Organizational Change}

Organizations looking to change may use a gap analysis that directs the organization to review where the organization is, identify where the organization would like to be, and note where there is room for improvement. "The type of support people need can only be determined after an analysis of what is required to close a specific gap and whether those required elements are readily available in the organization" (Clark \& Estes, 2008, p. 42). A gap analysis is one approach used to identify the organization's hierarchies, conflicts, and values; the organizational frameworks synthesized by Bolman and Deal (2008) are another. In their synthesis of research about organizations, Bolman and Deal categorized organizational issues. They argued that problems within organizations could be viewed in terms of four lenses or frames: structural, human resources, political, and symbolic.

The four frames approach partially matches the organizational lenses of Hatch and Cunliffe (2006). They categorize organizational theory into three perspectives: modernism, postmodernism, and symbolic interpretivism. Bolman and Deal's structural frame mirrors Hatch and Cunliffe's label of modernism as organizations focus on the effectiveness of their rules, policies, and procedures. The postmodernism perspective, enacting power relations and setting agendas, is best captured within Bolman and Deal's

political frame. As for the other two frames, human resource and symbolic, Bolman and 
Deal separate them. The two frames were initially together in Hatch and Cunliffe's symbolic interpretivism perspective. This perspective emphasizes that organizations are webs of meaning that display the spirit of the organization.

Four frames categorization of organizational theory. In order to address challenges of assessment reform, the four frames from Bolman and Deal (2008) will be examined. The structural frame uses the metaphor of a factory. Constantly managing effective turnover of products, a factory depends upon a goal-oriented environment incorporating rules, procedures, and policies. "The structural frame both enhances and constrains what an organization can accomplish" (Bolman \& Deal, 2008, p. 50) within hierarchies of authority. Focusing instead on relationships, the human resource frame is identified as a family. This frame uses motivation to address particular needs, feelings, and skills of the organization's workers to recognize the organization's strengths and weaknesses. According to the human resource frame, "the most important asset is the people” (Bolman \& Deal, 2008, p. 117).

The symbolic frame identifies the symbols, rituals, and stories that unite people to each other, but also instills pride within the organization. "The soul of the organization can also be viewed as a sense of character, a deep confidence about who we are, and what we care about, and what we deeply believe in" (Bolman \& Deal, 2008, p. 400). The political frame, or the jungle, typically carries a negative connotation. Even though the political frame utilizes power in situations, contests, or conflicts, the frame's positive side pinpoints the goals of stakeholders and holds a position to negotiate and bargain for those goals. "Once you cultivate the cheerleaders, the organization can move to promising rewards in exchange for resources and support" (Bolman \& Deal, 2008, p. 219). 
The frames guide organizations through high stakes circumstances (Bolman \& Deal, 2008). The possibility of transforming an organization relies on using one of the frames effectively. Exercising the questions written by Bolman and Deal (2008, p. 317), the political frame was identified as the best frame for effective assessment reform at District Alpha. Two of the questions distinguished the political frame choice as the most obvious candidate. The answer to the first question was that the organization will attempt to work bottom up. The answer to the second question was that there will be high levels of ambiguity. Both answers frame the study of parental support and assessment reform well. Parents respond better to problem solving with school districts when they are included in the process. As this was the first attempt of District Alpha to include parents in any reform situation, the study had high levels of ambiguity.

Issues in managing effective change. Real change in organizations is messier than the people in those organizations want to believe (Rost, 1993; Fullan, 2001). Other opinions regarding change include that change is difficult (Johnson, 1998), and there needs to be a compelling case for change (Senge, 2000). Initiating change in a school must improve student achievement; therefore, communicating change to stakeholders must have a focus based on relevance, readiness, and resource (Bridges, 2003; Fullan \& Hargreaves, 1991). "Cultures affect productivity, how well teachers teach and how much students learn by projecting an image of what the schools stands for, culture affects perceptions and confidence of parents and the community" (Deal, 1985, p. 611).

Hanson (2001) translates culture as community capacity, the organizational memory and organizational learning that must interact in order to bring about change. Gold, Simon, and Brown (2005) address the memory and learning of the community as 
the interaction between developing leadership, building new relationships within the community, and addressing community concerns. Ultimately, interactions within community capacity affect public accountability: "the hinge of change resulting in commitments that obligate parents, educators, community to follow through on their promises to improve schools" (Gold, Simon, \& Brown, 2005, p. 247). Byrk (2010) highlights the significance of the school improvement side with a school climate centered upon student-learning and a strong relationship between the school and community. The political frame, from Bolman and Deal (2008), adds to the discussion from Gold, Simon, and Brown (2005). The frame's barrier to change is the conflict between winners and losers: i.e., community powers. However, the frame's essential strategy is creating new coalitions, or in other words, addressing the challenge of public accountability by drawing in additional partners.

\section{Comparing Change Models}

"It takes about three years to achieve successful change in student performance in an elementary school. Depending on size, it takes about 6 years to do so in secondary schools" (Fullan, 2000, p. 581). With such a long time needed to address change, there must be suggestions for implementing change efficiently. Change models can be the 'how to' response to deal with change. Models provide practical steps ensuring that public accountability is addressed. The models provide a checklist that change agents must recognize and respond to. The following section will focus on two change models, what the two models have in common, what makes them different, and what component is missing in both. 
Kotter and Cohen (2002) model. The first change model is Kotter and Cohen's (2002) eight stages. This model provides suggestions for a successful change process by seeing, feeling, and then changing peoples' behavior (p. 8). The stages include:

1. Increasing urgency - When a sense of urgency is established, it will get people ready to be a part of the solution.

2. Building a guiding team-Having a lead team with the needed skills, credibility, and authority will move things along leading to a trusting, emotional commitment.

3. Setting the vision right-Designing an uplifting vision and compatible strategy with the pace of the organization will sustain change.

4. Communicating the buy-in-The vision and strategy should be communicated through a combination of words, deeds, and symbols. It's simple and direct. Repetition is key.

5. Empowering action-Leaders should remove obstacles, thus empowering people to move ahead.

6. Creating short-term wins - Just like with any athletic team, having a winning streak builds momentum and confidence. Leaders should encourage the small victories.

7. Don't let up — Stick to the plan and refuse to quit, even when the situation gets tough.

8. Making change stick-If the task is worth starting, then put resources in place to support and nurture a new innovative culture.

The Kotter and Cohen model appears linear, but this is not necessarily the case. It is possible for the stages to be implemented out of order or even repeated. It is often the case that this model will cycle back through and re-address one of the stages in order for the change to be more effective. Changing peoples' behavior is a challenge and should be addressed delicately.

Hall and Hord (2010) model. A second change model is the Concerns-Based Adoption Model (CBAM). CBAM describes change as an evolution through ideas of 
innovation, stages of concerns, and levels of use (Hall \& Hord, 2010). The detailed approach helps to define change as a very personal process. The Stages of Concern $(\mathrm{SoC})$ address perceptions of individuals progressing through the challenges of implementing innovation. The Stages of Concern include:

Stage 0: Unconcerned-Individuals do not know anything about the innovation.

Stage 1: Awareness - Individuals are now aware of the innovation; thus, the specifics of the innovation are necessary to know.

Stage 2: Personal-With knowing the facts, the individual questions how the innovation impacts them. The innovation now needs to address different perspectives.

Stage 3: Management-Once involved, the challenges of managing and organizing effective change emerge.

Stage 4: Consequence-The innovation needs to respond to the possibility of both positive and negative consequences that may undermine sustainability.

Stage 5: Collaboration-The call for reinforcements is completed through collaboration.

Stage 6: Refocusing-The innovation is working, but it can get better. The opportunity to brainstorm solutions emerges.

\section{Viewing Change Models through the Bolman and Deal Frames}

Both the CBAM and the Kotter and Cohen model can be viewed using the four frame lens from Bolman and Deal. Addressing people factors (Hall \& Hord, 2010, p. 15), the attitudes, feelings, concerns, and beliefs of the stakeholders are the human resource frame. Referencing the structural frame, both models agree that change is a process, not just an event. "Change is a process through which people and organizations move as they gradually learn, come to understand, and become skilled and competent in the use of new ways" (Hall \& Hord, 2010, p. 8). Change is collaborative, as both models 
discuss teamwork through guiding teams (Kotter \& Cohen) or professional learning communities (Hall \& Hord). These resource groups refer to the identifiable groups within the political frame. Finally, throughout the change process, leaders provide intervention (Hall \& Hord) and remove obstacles (Kotter \& Cohen). In some cases, the intervention or obstacle may be the symbol to help humans make sense of the change process.

There are a few differences between the models. CBAM is a more linear process, suggesting that change cannot move forward until the concerns are met at the lower levels. On the other hand, it is suggested that the Kotter and Cohen model is cyclical. In Kotter and Cohen, the guiding team needs to inspire others to get involved, while in CBAM, an administrator's leadership is essential for long-term change. This difference is due to the CBAM being more specific in addressing change within schools; however, both models ignore transitions. Change focuses on the outcome, whereas transition means leaving the situation behind. In order to make a change or reform long-lasting, it is important for leaders to understand that an unmanaged transition can make the change unmanageable (Bridges, 2003, p. 146). The best way to manage transitions, and therefore change, is for those initiating reform to be able to answer this question, "Who is going to have to let go of what to make the change work as planned" (Bridges, 2003, p. 146)?

Professional development supporting change. According to Shepard (2000), there are two fundamental pillars of assessment change. First, proposed assessment change must be altered to better represent important thinking and problem-solving skills. Secondly, the execution of assessment change in the classroom as well as its reception by teachers and students must change. Elmore (2007) closed the gap between performance 
expectations and the people meant to achieve these expectations. Effective professional development must be designed to improve the skills and knowledge of its educators (Elmore, 2007). Professional development is the transition after change that can support the endeavor. Baker, Curtis, and Benenson's (1991) four sectioned diagram of 'meaning of planned change' can guide the effectiveness of professional development within schools.

The most promising of the four change expectations is collaborative opportunity. Simply put, this is working together. It is most effective professional development, since all parties involved share purpose and mutual commitment (Sparks \& Loucks-Horsley, 1989). This is also where relational trust between administrators and teachers plays a large role as both sides must work together (Bryk, 2010). If the opportunity includes ongoing coaching and frequent feedback, it results in a $90 \%$ transfer into practice (Joyce \& Showers, 2000). Mandated opportunity displays the leader as committed to change and mandates others to participate in professional development to support the change. It may take several years for all teachers to be involved in professional development activities, but through observations and reflections, it will become an authentic opportunity (Sparks \& Loucks-Horsley, 1989).

Another avenue of planned change interpreted by Baker, Curtis, and Benenson (1991) is compliance. In collaborative compliance, the leader approaches professional development collaboratively via committees; however, the teachers attend out of obligation and go through the motions of being interested. It appears to be collaboration but in this scenario, only $20 \%$ transfers into practice (Joyce \& Showers, 2000). Finally, within mandated compliance, the leader takes a more authoritative role and anticipates 
full participation in professional development since the teachers are capable of selfinitiated learning (Sparks \& Loucks-Horsley, 1989). Unfortunately, the teachers have no commitment to professional development. Due to the teachers' procrastination, typically only 5\% transfers into practice (Joyce \& Showers, 2000).

In sum, the best implementation for professional development to support an assessment change must include a demonstration of the desired skill, provide on-going focused feedback, and set aside time for reflection (Hillard, 1997). Accountability must be a reciprocal process (Elmore, 2007, p. 93). The reciprocity must be included within any professional development within the school. Therefore, the best model, designed by Baker, Curtis, and Benenson (1991), in managing accountability is the collaborative opportunity.

\section{Leading Through Change}

"Leadership drives change” (Bryk, 2010, p. 25). Gold, Simon and Brown (2005) agree with Bryk as their theory of change includes a leader who drives community capacity via the relationships with the community. For school improvement, the leader drives the local activities toward instruction. With leadership being a huge focus, the district must be ready to establish and maintain a clear focus on the future and behave strategically. Blankstein (2010), reinforcing Bryk’s declaration, states,

School systems will have to acknowledge and create conditions that distribute leadership far beyond the head teacher's office to the entire culture of the school...And they will need to concentrate on the leadership skills and qualities that will sustain leaders into the future rather than merely help them manage and survive in the present. (p. 213)

Leadership is understood, not just as a possession of the leader, but as an aspect of the community (Goldberg, 2006). Leadership is framed by communal capacity and 
communal achievement (Drath, 2001). Nurturing an aspect of the social justice framework, leaders must "create a culture that embraces change and decision making in collaboration with parents and other stakeholders" (Mullen, 2010, p. 333). Lastly, leadership is "an influence relationship among leaders and their collaborators who intend real changes that reflect their mutual purpose" (Rost, 1993, p. 99).

Rost (1993), Drath (2001), Mullen (2010), and Goldberg (2006) affirm that the key to leadership is working together, but Cohen and Bradford (2005) contend that collaboration does not just happen. Cohen and Bradford suggest that to address change and collaboration, leaders must understand the "exchange of currency." Currency explains how groups of people accomplish their tasks through motivation, inspiration, or consultation (Cohen \& Bradford, 2005, p. 57). Cohen and Bradford's currency model provides a backdrop to contrast the different leadership approaches. Relationship-related currency is a response to the desire to belong to a group. With the strength of relationships being important to this currency, servant leadership becomes a good match, since its asset is listening to the concerns and issues of others. Inspiration-related currency provides meaning for the work people do. Its currency definition is best suited to the transformational approach since the objective is to inspire and discover value. Task-related currency is a more organized approach to get work done by increasing skills and abilities of others, thus coordinating with adaptive leadership. Finally, positionrelated currency addresses how individuals improve their ability to do work and discover opportunities to network with others. A comparable leadership style is distributed. 


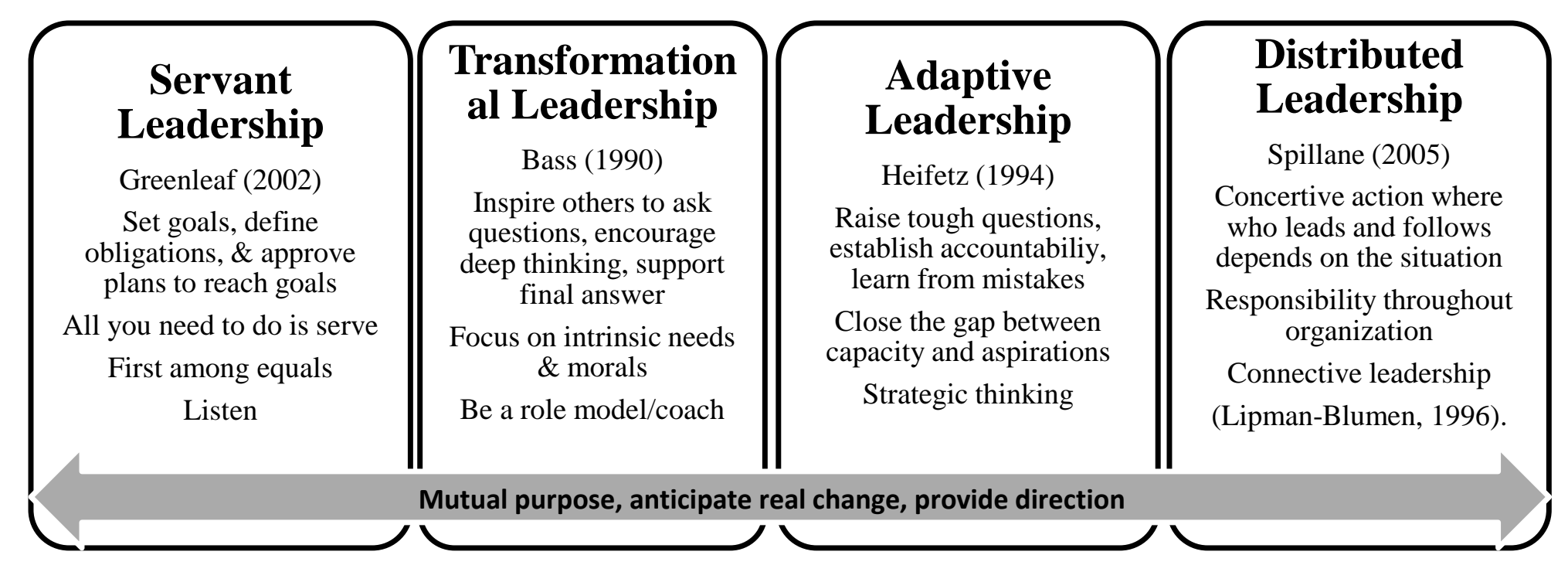

Figure 1. Comparing and Contrasting Leadership Approaches 
Figure 1 represents each leadership approach with its major advocate and characteristics. Educational change within schools should strengthen student performance, thus improving student achievement; therefore, it is important for leaders to possess particular elements:

- To achieve mutual purpose (Rost, 1993) and be able to read the situation (Huckaby, 1980)

- To gain knowledge and understand the relationship between the demands and the call to lead (Goldberg, 2006)

- To anticipate real change (Rost, 1993), by setting priorities as goals (Goldberg, 2006), and giving specific direction (Drath, 2001)

\section{Leadership approaches to involve parents in assessment reform. Out of the}

four leadership approaches, some are more similar to each other. Adaptive and distributed leadership styles are more like the structural frame approach because they focus on the organization itself. On the other hand, transformational and servant leadership are more relationship based and nurturing, representing the human resource frame. Each approach has characteristics that do identify well with the assessment reform targeting support from parents.

A sense of community is most important in servant leadership. Stressing that leaders just need to serve, leaders are the "first among equals" (Greenleaf, 2002, p. 74.) For this particular assessment reform, this approach may be too soft and too unexpected. Even though District Alpha is a proponent of servant leadership, parents are unfamiliar with seeing it in action. However, the listening component of servant leadership is what is necessary in dealing with parents' perceptions of assessment. "Nothing is meaningful until it is related to the hearer's own experience" (Greenleaf, 2002, pp. 31-32). A similar nurturing behavior is seen within transformational leadership. Transformational 
leadership addresses its nurturing manner as a coach or role model for a group. The coach will help the group make a shared decision by remaining optimistic, focusing on intrinsic motivation, while staying the identifiable leader. The Blueprint for Reform argues that transformational leaders provide the necessary skills for turnaround efforts in schools, including effectively engaging families and community members (Department of Education, 2010a). The coaching attribute is appealing to assessment reform with parents; however, having no previous experience, I am unsure of what parents will need. Adaptive leadership may be a better approach to identify with the assessment reform targeting support from parents. Adaptive leaders "influence the environment toward a desired direction, mobilizing people to tackle tough challenges" (Heifetz, Grashow, \& Linsky, 2009, p. 78). This approach allows the leader to stay knowledgeable on what stakeholders want with cooperation highly regarded. Adaptive leadership remains committed to the community and allows for more trial and error. However, the 'holding feet to the fire' accountability method may not be best to spark collaboration with parents. Instead, the distributed approach may be the answer, as it is the responsibility of the organization to solve the problem. Spillane, Halverson, and Diamond (2001) affirm that distributed leadership is the most appropriate for studying leadership practice in schools. Depending upon the situation and the problem being tackled, the role of the leader emerges from anyone involved (Copland, 2003, p. 378). The concerted effort of those involved is "a collective phenomenon where leadership is present in the flow of activities in which a set of organization members find themselves enmeshed" (Gronn, 2000, p. 331). This practice allows distributed leadership to be seen as a 'snowball effect', the works of others exponentially growing for a good cause. 
Returning to the political frame conversation, Bolman and Deal (2008) link the reframing of organizations with reframing leadership. Since the political frame was identified as most applicable to assessment reform, the political leader plays an advocate role where leadership focuses on building coalitions (Bolman \& Deal, 2008). Political leaders reach out to the community and work with key stakeholders to identify and build common interests and relationships, for a political leader's “influence begins with understanding others' concerns and interests” (Bolman \& Deal, 2008, p. 366).

\section{Assessment Practices Past and Present}

If we are finally to connect assessment to school improvement in meaningful ways, we must come to see assessment through new eyes. (Stiggins, 2002, p. 1)

Even though educational reform was enacted before 1965, it was the Elementary and Secondary Education Act (ESEA) that has been most instrumental in creating the standards-based reform that we see in 2014. The ESEA was the first uniform approach tackling equality for all students while establishing a need for high standards and accountability. Ironically, the act forbade the establishment of a national curriculum. The lack of ownership in advancing American schools may have been the underlying theme of the Nation at Risk report outlining the reasons why America was not producing a world competitive student.

Goals 2000: Educate America Act was Clinton's attempt to redirect schools in the early 1990s. "By the year 2000, students will leave grades 4, 8, and 12 showing competency in subjects and the schools will ensure all students learn to use their minds well" (Goals 2000, 1994). Unfortunately, another condition within the Act was that the United States would rank first in the world in mathematics and science achievement, and 
that is yet to be a reality.

\section{Learning Standards as Reform}

To increase accountability to the learning standards, the No Child Left Behind (NCLB) Act of 2001 stressed annual yearly progress (AYP) and increased percentages of students meeting standards in order to receive any federal funding. With the threat to earmark particular school districts as failing, districts began to focus on how to show improvement and choosing a 'teach to the test' approach, instead of incorporating more student engagement in their learning. Standards-based reform took on a bad reputation. Questioning of the credibility of the standards, especially in assessing the knowledge and skills of the students on those particular standards, became a concern.

The most recent adaption of the ESEA is Obama's Blueprint for Reform. Within this reauthorization, standards and assessments are aligned to college readiness standards, thus creating a more well-rounded education opportunity and clear expectations. The accountability structure created by No Child Left Behind morphed into the Race to the Top incentive program where districts may be rewarded monetarily when their schools show improvement or innovation. Today's education is standards-based with emphasis on accountable assessment practices. Next generation learning, such as Common Core and Next Generation Science Standards, prepare students for "the acquisition of knowledge and skills while engaging students in their educational experience" (Weiss, Lopez, \& Rosenberg, 2010, p. 4).

It is not surprising that the Center for Educational Reform gave the state of Illinois a "D" for its weak performance on their environmental criteria category for an effective school. From a study conducted by Advance Illinois (2010), the poor grade is 
due to the lack of longitudinal data, the delay in reevaluating teacher and principal effectiveness, and, most importantly, the need for better ways to assess student readiness. One prerequisite to assessing readiness is setting clear expectations. Highlighting standards-based education again, it is important to have a yardstick to measure success. Even though Illinois initially created learning standards for the major subjects (English, math, science, social science) as recently as 1997, Illinois embraced the opportunity to readdress learning standards. During the creation of Common Core, Illinois was a lead state and officially adopted these new standards for English and Math in June 2010. In addition, Illinois recently became the eleventh state to adopt the Next Generation Science Standards in March 2014. These new standards aim to provide clear, consistent, academic benchmarks with "fewer, clearer and higher academic standards for essential learning and skills" (Illinois State Board of Education, 2012).

\section{Incorporating Assessment into Reform}

Along with placing emphasis upon standards, Illinois has also made a push to include a better assessment system than the one developed to manage NCLB. For the past decade, Illinois has administered the ACT and WorkKeys program (incorporated together as the Prairie State Achievement Exam) to all eleventh graders as part of the state's assessment system. The new assessment piece, planned to be in place 2014-2015, utilizes the Partnership for Assessment of Readiness for College and Careers (PARCC) tests. The PARCC assessment system involves more performance based assessment opportunities for grade levels $9-11$ via a computer to transpire at the $75 \%$ and $90 \%$ mark of the school year. Using Race to the Top funds, this assessment system will evaluate if students are ready for college-level coursework and measure the full range of skills in the 
common standards.

\section{Comparing Assessment of Learning and Assessment for Learning}

Black and Wiliam (1998) advocate the need to change assessment practices in order to strengthen student learning. The pinnacle work of Black and Wiliam, later supported by Hargreaves (2005), defines formative assessment as activities completed within a process of feedback to direct where learners are in their learning, where they need to go, and how best to get there (Black \& Wiliam, 1998; Hargreaves, 2005). The evolution of formative assessment began with Black and Wiliam's (1998) claim that the most important difficulties with assessment revolve around three issues.

Issue one. Issue one is effective learning, returning back to a discussion of the standards-based learning environment. The argument is between the quality and the quantity of effective learning. There is a tendency for educators to emphasize the quantity of standards. There are always too many standards in too short a time. It is the old cliché: it's a mile wide and an inch deep. There is a gap in addressing obtained skills learned throughout a course, especially in a science course, and promoting independent thinking.

Issue two. The second issue of assessment is its negative impact. The use of standardized tests is meant to demonstrate the progress made through standards-based learning. Critics such as Popham (2007) state that standardized tests are "instructionally insensitive," meaning that performances on these tests do not reflect the quality of daily instruction (p. 146). High-stakes standardized tests elevate competition instead of applauding the personal accomplishment and improvement a student made during his or her educational journey. The message that school is about learning is blurred (O'Connor, 
2011). Assessment is a tool for monitoring, and its tasks should reflect the progress made through learning (Carnoy \& Loeb, 2002). Unfortunately, standardized tests, such as PSAE, diminish the positive impact of formative assessment. Instead, emphasis is placed on the school's performance on such tests promoting the difference between the academic 'haves' and 'have nots.' Focus should be placed on a belief that all pupils can achieve (Black \& Wiliam, 1998).

Issue three. The last issue of assessment is the managerial role of assessments. As already mentioned, the political commitment to summative assessments is a strong force. These assessments are known to parents as the method to check in, to compare, and to rank student learning according to defined standards, or to other districts or students (Guskey, 2006). Parents anticipate a similar report card of their student's academic performance with an alphabetical display. The tradition of multiple choice questions to evaluate learning is challenged and places more accountability with the teachers and parents.

Table 1 summarizes the differences between 'assessment of' and 'assessment for' learning. Summative assessment, or assessment of learning, has been more dominant as it indicates the effectiveness of student learning at the end of a learning period. Edwards, Turner, and Mokhtari (2008) describe assessment of learning as the assessment done to students rather than with students. On the other hand, working with students through a process to continuously provide students with the chance to evaluate the effectiveness of their learning is $\mathrm{A} 4 \mathrm{~L}$, the formative approach to assessment. 
Table 1

The Differences Between Summative and Formative Assessment

\begin{tabular}{|c|c|c|}
\hline & Assessment of Learning & Assessment for Learning \\
\hline Assessment form & Summative & Formative \\
\hline Primary users & Teachers, school district, state & Teachers, students \\
\hline Assess What & $\begin{array}{l}\text { Standards, benchmarks, or } \\
\text { curriculum objectives }\end{array}$ & $\begin{array}{l}\text { Explicit learning targets used in } \\
\text { the course curriculum }\end{array}$ \\
\hline Assess How & Multiple choice or short answer & $\begin{array}{l}\text { Variety of methods best matching } \\
\text { the learning target (i.e., portfolios, } \\
\text { presentations, labs) }\end{array}$ \\
\hline Assess When & $\begin{array}{l}\text { An event after learning, } \\
\text { periodically or annually }\end{array}$ & $\begin{array}{l}\text { Continuous, on-going, a process } \\
\text { during learning }\end{array}$ \\
\hline Typical Users & $\begin{array}{l}\text { Communicate level of perform- } \\
\text { ance against statistics, measure } \\
\text { achievement at particular } \\
\text { points, aid in decisions } \\
\text { regarding district resources }\end{array}$ & $\begin{array}{l}\text { Support learning, reflect on } \\
\text { progress made toward objectives, } \\
\text { adjust instruction, provide } \\
\text { descriptive feedback, enable } \\
\text { students to engage in their learning }\end{array}$ \\
\hline $\begin{array}{l}\text { Student involvement } \\
\& \text { motivation }\end{array}$ & Discouraged, extrinsic & Encouraged, intrinsic \\
\hline Effect on learning & Weak, fleeting & Strong, positive, long lasting \\
\hline
\end{tabular}

Note. Adapted from McMillan (2007) and Paine (2008).

The five key strategies for effective formative assessment consist of:

1. Clarifying learning intentions and sharing criteria for success.

2. Engineering effective classroom discussions, questions, and learning tasks that elicit evidence of learning.

3. Providing feedback that moves learners forward

4. Activating students as the owners of their own learning

5. Activating students as instructional resources for one another (Wiliam, 2007, p. 192)

These strategies are identified within the assessment for learning paradigm. Assessment as learning gives priority to student learning (Black \& Wiliam, 1998; Black, Harrison, Lee, Marshall, \& Wiliam, 2004) and views mistakes as the beginning of learning (Guskey, 2003; Wiggins, 1998). The A4L process does not provide ranks of competence; 
instead, A4L provides opportunities for students to assess themselves through a formative process. Formative assessment is based on short-cycle assessments providing bite-sized chunks of feedback, noted as tactic adjustments for both teachers and students, used as evidence of the students' mastery of knowledge and skills (Black \& Wiliam, 1998; Stiggins, 2005a; Popham, 2008). Assessment for learning is a method remaining committed to standards-based instruction (Stiggins \& DuFour, 2009), but it should not be viewed as this year's educational fad (Popham, 2006). Over time, if schools target the proper implementation, invest in teacher training, and nurture the A4L goals, the statistics as reported by Black and Wiliam (1998) are "amongst the largest ever reported for educational intervention" (p. 61).

"The key to success is finding the synergy between the two [assessment] concepts" (Stiggins, 2007, p. 70). Even though standards-based learning, such as Common Core, has placed summative assessment under the microscope, it is important to recalibrate to include an equal representation between both assessment types. Just measuring a student's demonstration of discrete facts is faulty especially in more complex situations when the student must apply facts and concepts. Data collected from various sources can reveal how teachers can improve their teaching and ultimately create opportunities to include assessment for learning (Stiggins, 2002). When creating an executive summary designed to help address goals of assessment of the Common Core standards, the Educational Testing Service (ETS), Pearson, and College Board resonate with Stiggins (2002, 2007).

Summative assessments will remain a key element of an educational quality management system, and one of the main goals of this effort is to improve the quality and efficiency of our summative system. However, without questioning 
this goal, we believe that American education would be best served by an integrated system where summative and interim formative components are built from common frameworks. (ETS, 2010, p. 7)

The $21^{\text {st }}$ century learning skills framework sparked the creation of the Common Core standards. Within the framework are skill categories of learning and innovation, information and technology skills, and life and career (Partnerships for $21^{\text {st }}$ Century Skills, 2009). The skills listed under the life and career category best address the goals of Assessment for Learning (A4L). Incorporating feedback effectively and self-monitoring are the $21^{\text {st }}$ skills that persuade districts to advance the assessment for learning paradigm.

Targeting active student learning. Instructional strategies of assessment for learning include providing daily learning targets, offering descriptive feedback, and engaging students in self-reflection (Chappuis, 2005). If educators can help students "see, understand, and appreciate" their academic journey, then students will find their true selves without outside expectations (Stiggins, 1999). For Black and Wiliam (1998), self-assessment is the key component of formative assessment. Stiggins (2002, 2005b) refocuses his assessment for learning concept to include building a healthy assessment environment centered on motivation supporting the practice of self-assessment.

Self-assessment is a process during which students personally reflect on their personal perceptions and predispositions of learning and evaluate the quality of their work by identifying strengths and weaknesses (Andrade \& Du, 2007; Stiggins \& Popham, 2008). Improvement made by students between their first attempt and the last is the goal of self-assessment (Wiggins, 1998). According to Chappuis and Chappuis (2008), improvement finds success when students address these questions in their selfassessment practice: 
- What are my strengths relative to the standards?

- What have I seen myself improve at?

- Where are my areas of weakness?

- Where didn't I perform as desired, and how might I improve those answers?

- What do these results mean for the next steps in my learning, and how should I prepare for that improvement? (p. 15)

Teachers also have an important role in self-assessment by providing feedback. If teachers provide positive feedback, then assessment becomes integrated into how students learn, thus improving student achievement. Feedback regarding student effort has three elements: redefinition of the desired goal, evidence about present position, and some understanding of a way to close the gap between the two (Black \& Wiliam, 1998, p. 85). In order for feedback to be meaningful, feedback must be accurate, timely, and specific (Reeves, 2007, p. 228). Consider any athletic team; how would the team perform without a coach providing feedback? Effective feedback not only tells us how we perform, but how to improve the next time. This too is an important part of the assessment for learning paradigm.

\section{Challenges of Assessment Reform}

Proper implementation of student self-assessment can be accomplished with time and practice to develop the skill (Guskey, 2003; Black, Harrison, Lee, Marshall, \& Wiliam, 2004). Even while citing an overall positive self-assessment experience, middle school students mention an environment of tension and stress (Andrade \& Du, 2007). From the viewpoint of participating students, teacher expectations were not communicated well, thus making full participation in self-assessment activities difficult. Corrective instruction is not the same as reteaching (Guskey, 2003). Once teachers are more aware of the power of assessment, the self-assessment will become more effective. 
Andrade (2011) warns that self-assessment is not glorified self-reflection. The practice of self-assessment is most useful when utilized during the learning process, not just at its conclusion. Grounded in formative assessment, assessing one's self is a process through evaluating curriculum objectives or learning targets. Students need to be aware of the benefits of self-assessment and must be able to identify learning targets in order to be successful in the process (Andrade, 2011). The last suggestion by Andrade (2011) echoes comments from Guskey (2003), Black, Harrison, Lee, Marshall, and Wiliam (2004) that self-assessment takes practice.

Cultural adaptation. Boyle and Charles (2010) assumed, over the 6-year nationwide implementation period of assessment for learning in England, that English schools earned total participation from its teachers. Unfortunately, Boyle and Charles revealed that assessment for learning was not totally immersed into the schools. One conclusion made was that the shift toward a different assessment practice is difficult. Many educators still stand behind a 'one size fits all' mentality in regard to assessment and its practice. Even though Black and Wiliam (1998), Stiggins (1999), and Marzano (2003) support an assessment change benefiting students, educators drag their feet when it comes to change. Even Black and Wiliam, with a new team of researchers, reviewed the shortcomings of their initial study. Black, Harrison, Lee, Marshall and Wiliam (2004) emphasize that current assessment methods still do not promote learning, grading practices emphasize competition instead of self-achievement, and any assessment feedback is negative. Webb and Jones (2009) explored the tensions of elementary school teachers in regard to implementing the assessment for learning initiatives in their classrooms. Finding similar results to Boyle and Charles' (2010) study, Webb and Jones 
(2009) note that even though formative assessment is desirable, it is not easy. The school's paradigm stands in the way.

If educators are not convinced during assessment change, then it is possible that parents may show some resistance as well. Blankstein (2010) identifies obstacles associated with change and comments that parents want their children's school days to be just like their own. Typically, if parents refer back to their own education experience, it is due to the lack of communication between the school and the parents (Mu \& Childs, 2007). Before Boyle and Charles' (2010) research on the assessment for learning concept implemented in English primary schools, Carless (2005) notes similar resistance to changes made in assessment practices in Hong Kong. In both studies (Carless, 2005; Boyle \& Charles, 2010), attempts made at reforming assessment practices failed. Failure in Hong Kong and England is due to the lack of consensus on the value of assessment. The lack of consensus is the communication barrier between improving assessment practices and adapting the culture. Communication to parents is critical, and Webb and Jones (2009) urge more parental communication to aid in changing classroom instruction. Parents need to know that cooperation and collaboration from both school and home is essential to build capacity to deliver better education (Carnoy \& Loeb, 2002). Communication regarding assessment cannot be on the defensive, answering to the comment or complaint of every 'helicopter' parent. Their constant hovering and divulging over matters of the school must be faced proactively by displaying transparency.

Grading crisis. Buried within the dynamic of school culture is the challenge of grading, especially within formative assessment. Changing current attitudes toward grading is a major undertaking, especially at the high school level (O'Connor, 2011). 
Many high school symbols (valedictorian, salutatorian, top 10) depend upon grades. Grading typically promotes a culture of point accumulation, not learning. It encourages competition, stratifies students and punishes students who do not work hard (O'Connor, 2011, p. 127). Grades, as motivators, breed dependence, and reduce risk taking, creativity, and value (Stiggins, 2007). And many parents desire this disjunction of 'the haves' and 'the haves-not'. However, the focus should be placed on intrinsic motivation, supported by parent engagement in academics, to develop students into being independent, self-directed, lifelong learners.

"Students need feedback and lots of it, but grades are not the best forms of feedback" (Wormeli, 2006). A grading system that documents student progress, provides feedback to the student and parent, and informs instructional decisions is useful, worthy, and desired. By combining large-scale summative assessments of student learning with smaller in school formative assessments for learning, educators create more comprehensive representations of student progress (Stiggins, Arter, Chappuis, \& Chappuis, 2004). Shepard and Bliem (1995) reported that parents participating in their study appreciate formative assessment more than summative, dating before Black and Wiliam's metaanalysis in 1998. The formative assessments force students to think. Another benefit indicated by parents is that formative assessment provides evidence to the teacher as to whether students are understanding or struggling. However, the challenge is communicating formative assessment progress. Marzano (2007) suggests reformatting the report card to include progress of the student along with existing standards used for that course.

The next challenge of grading within the assessment for learning paradigm is the most controversial. It is the "no zero" policy. Since formative assessment highlights 
student progress, a zero is ambiguous. A zero can communicate either the work was not completed or the work did not show improvement. To eliminate confusion, it is suggested by O'Connor (2011) to use an 'I' to signal insufficient evidence. In regards to motivation, zeros are counterproductive. "A zero has an undeserved and devastating influence, so much so that no matter what the student does, the grade distorts the final grade as a true indicator of mastery" (Wormeli, 2006, p. 137). The zero kills the entire process of student self-assessment, since few students learn from an experience in which there is no hope for positive recognition of learning. Student accountability without purpose is one reason why students fail (Wormeli, 2006). Therefore, it is suggested to mark the missing assignment as a 50\%. The percentage is still a failing grade; however, it is more recoverable showing what the student truly knows. "If the purpose of grading and reporting is to provide an accurate description of what students have learned, then averaging must be considered inadequate and inappropriate" (Guskey, 1996, p. 21).

\section{Local Implementation of Assessment for Learning}

Ravitch (2010) declares, "If there is one consistent lesson that one gleans by studying school reform...it is the danger of taking a good idea and expanding it rapidly, spreading it thin" (p. 146). Ravitch should not include District Alpha in her analysis of school reform efforts. Starting with Dr. Borat's introduction of the Assessment for Learning (A4L) paradigm, District Alpha has slowly built momentum. Elmore (2002) points out that school plans should be continuously evaluated, and this evaluation should be based on the effect the action has on student achievement (p. 8). Table 2 displays learning strategies designed to mirror the key strategies for effective formative assessment (Wiliam, 2007). The strategies are more specific than the original five and 
include a student performance element, following Elmore's advice. The included percentages were from research conducted mostly by Marzano (2003, 2007). "Research will never be able to identify instructional strategies that work with every student in every class. The best research can do is tell us which strategies have a good chance (high probability) of working well with students" (Marzano, 2007, p. 5).

Table 2

Adopted Learning Strategies for District Alpha

$\begin{array}{ll}\text { Learning strategy } & \text { Implemented }\end{array}$

1. State and show learning objectives at the beginning and end of each Fall 2008 lesson.

Increase in student performance is $27 \%$

2. Explicitly teach vocabulary as well as retention and memory strategies Fall 2008 Increase in student performance is $33 \%$

3. Encourage student self-assessment and adjustment

Fall 2009

Increase in student performance is $24 \%$

4. Explicitly teach learning skills and strategies using summaries for

Fall 2010 patterning, thinking, and writing.

Increase in student performance is $34 \%$

5. Provide frequent feedback to students about their learning related to the Fall 2010 objective within 48 hours.

Increase in student performance is $37 \%$

6. Explicitly teach learning skills and strategies using graphic organizers Fall 2012 for patterning, thinking, and writing.

Increase in student performance is $27 \%$

7. Help student activate and build background information and advance Fall 2012 organization.

Increase in student performance is $22 \%$

8. Provide corrective and enrichment activities that respond to student

Fall 2012 progress and provide additional opportunities that allow students to demonstrate learning.

Increase in student performance is $27 \%$ 
Starting with the 2008/2009 school year, District Alpha eased toward formative assessment practices integrating these strategies into the district improvement plan. District Alpha offers professional development opportunities to address these goals during institute days, summer classes, and Friday morning meetings. The implementation process has been slow but that is deliberate. Administrators encouraged only what the teaching staff could implement well into their instruction. During the slower process, teacher understanding of the strategies rose. Both strategies begun in 2008 can be seen in most, if not in all, District Alpha classes. The district's adopted summarization and feedback strategies are maturing. Finally, strategies 6,7 and 8 will soon be incorporated in all classes with hopes of an easy conversion. These strategies have been piloted within the required freshmen reading seminar course and overall have been successful.

O’Connor (2011) states there are two givens that cannot be questioned in schools. The first is that all assessments must be of high quality. Secondly, students must be active in the assessment process. Engagement of students is the integral component to extend learning for a lifetime. Most proponents of the assessment for learning paradigm recognize the learning gap between what occurs in today's classrooms and Black and Wiliam's (1998) vision for better assessment practices. However, assessment has changed over time with either good intent or a knee-jerk reaction (Shepard, 2000).

Significant school transformations will require more than changes in structure; the policies, programs, and procedures of a school. Substantive and lasting change will ultimately require a transformation of culture-the beliefs, assumptions, expectations, and habits that constitute the norm for the people throughout the organizations. (DuFour, Eaker, \& DuFour, 2005, p. 11)

Today in education, the essential parts are available to improve student achievement. Tying curriculum and instruction together, common standards such as the Common Core 
and Next Generation Science Standards have been created. An awareness of balancing assessment to include more formative opportunities is building. It is now time to implement change and take a leadership role. Schmoker (2004) states, "Once the infrastructure for improvements includes common standards and assessments, the opportunity for effective leadership emerges" (p. 5).

\section{Parent/Family Engagement}

When it comes to a breakfast of ham and eggs, the chicken is involved but the pig is committed. (Ferlazzo, 2009)

One of the most recent federal education initiatives spotlighting the involvement of parents is the No Child Left Behind Act. Defined within the federal policy, it is "the participation of parents in regular two-way and meaningful communication involving student academic learning and other school activities" (NCLB, 2001). NCLB was a reauthorization of the 1965 Title I of the Elementary and Secondary Education Act (ESEA). The ESEA promoted professional development, resources to support educational programs, and parental involvement. A Nation at Risk destroyed the momentum of the ESEA. The widely discussed publication shifted the focal point from the child's family to a competitive focus on standardized tests at each transitional point in schooling. Beginning in the 1990s, educational politics debated the emphasis of education's link to the global market. Worried that education was becoming more linked with federal control (Spring, 2005), family values resurfaced. Therefore, the Goals 2000: Educate America Act stated, "By 2000, every school will promote partnerships that will increase parental involvement and participation in promoting the social, emotional, and academic growth of children" (Carreon, Drake, \& Barton, 2005, p. 467). 
Presenting values reinforced in the home may be the next step for parent engagement in schools. The new approach for school districts, according to the Blueprint for Reform, is "to create a welcoming environment using open communication, family engagement, and allow states to support, identify, and disseminate best practices from activities funded by the Family Engagement and Responsibility fund" (Department of Education, 2010a). The Blueprint for Reform policy is designed to create a better rounded education instead of a system that focuses on standards. Family engagement can no longer be "treated as a discrete activity but an integrated strategy" (Department of Education, 2010b, p. 1). The policy intends parental involvement to be a long-term solution to academic success instead of a short-term one. By maintaining a shared vision, the policy stresses a collaborative effort between federal, state, and community agencies as well as school districts to improve family engagement, empowerment, and responsibility.

It is the responsibility of schools and teachers to develop and implement appropriate partnership practices at each grade level (Epstein, 1995). The national statistics of parent involvement does highlight a decrease from elementary to secondary schools. In 2007, almost $90 \%$ of students in kindergarten through fifth grade had parents attend a meeting with their teachers compared with $76 \%$ of middle-school students, and $61 \%$ of high school students (Child Trend Data Bank, 2012). Once again, these numbers demonstrate that strategies used in elementary districts do not last once those students are teenagers (Hill \& Chao, 2009). For public schools in Illinois, parent involvement is outlined within the school's report card as a percentage of students whose parents have had "personal contact including parent-teacher conferences, parental visits to school, school visits to home, telephone conversations, and written correspondence" (Illinois 
State Board of Education, 2009) during a school year.

The average parent involvement value for District Alpha's elementary district is $100 \%$, while the District Alpha's average is $97.9 \%$ (Smith, 2011). Both districts are above the state average of $96 \%$ for parent involvement. The potential to close the gap in the decline of parent involvement in high school as well as exercise parents as resources is at the fingers tips of the school administrators. Illinois' usage of parent involvement ignores studies on the process of parental engagement by Epstein $(1992,1995,2005, \&$ 2011). Epstein, and also Weiss, Lopez, and Rosenberg (2010), stress that educators must reduce their tendency to treat parents and family as bystanders but instead, increase the capacity to become partners. Auerbach (2009) continues to provide insight into the challenges of maintaining and creating parent engagement as she highlights essential steps administrators must take to promote meaningful family engagement in schools. In a previous study, Auerbach (2007) established that administrators believe parent engagement is a tool for raising student achievement.

Within this section of parent/family engagement, the literature will stress the fact that support from parents and family does make a difference in the learning of teenagers. With the years of research supporting how the home benefits academics, it is likely that the objectives of the Assessment for Learning (A4L) program will also be supported. Before addressing those benefits of parent engagement and support in the learning of teenagers, it is important to clarify differences between involvement and engagement. Schools are looking for sustained support in reform; it is important to avoid the random acts of family involvement (Weiss, Lopez, \& Rosenberg, 2010). 


\section{Transition from Involvement to Engagement}

Ferlazzo (2009) proposes that pointing out the difference between parent involvement and parent engagement is like comparing breakfast items. Both eggs and ham are useful breakfast items; however, for the chicken, producing eggs is routine, while the pig must be sacrificed to make ham possible. The same goes for parent involvement (the chicken) and parent engagement (the pig).

Defining involvement and engagement. The task of reaching out to the community is imminent. However, the correct terminology for involving parents has prevented schools from moving forward: is involvement or engagement desired? Similar to the definition used on Illinois school report cards, parent involvement, defined by Hill and Taylor (2004), consists of activities such as volunteering at schools, communicating with teachers, assisting with academics at home, and attending school events. For years, the terms involvement and engagement have been considered interchangeable, since both terms describe a relationship necessary to support the educational and academic lives of children. Noting the difference between the terms, Ferlazzo (2011) claims involvement as 'doing to' versus engagement 'doing with'. Chadwick (2004) notes that the choice in communication indicates the goal for the district. Involvement consists of words such as product, plan, and telling, whereas engagement includes words of process, vision, and sharing. Auerbach (2009) is fluent in stressing the difference between engagement and involvement. Engagement is more than reviewing homework or attending athletic events. Engagement develops opportunities that shape the needs of a school community. Ferlazzo and Hammond's (2009) definition targets engagement as "harnessing their (parents') own energy" (p. 8) to create an enduring partnership. 
Partnerships are characterized by trust, listening, and shared decision making (Marsh, 2007; Ferlazzo, 2011). Listening to the wisdom that parents have gained in more than 14 years of raising their children may benefit reform efforts in high schools; however, it is risky to value the knowledge/experience of the parent over the knowledge/experience of the educator. Auerbach (2009) observed a quote posted above the entrance to an administrator's office which is applicable to the significance between involvement and engagement: "Nothing is more important to success in schools than relationships between and among students, staff, and parents" (Auerbach, 2009, p. 19). Family engagement is a shared responsibility (Weiss, Lopez, \& Rosenberg, 2010) anchored in the efforts to improve the quality of education for all children (Mapp, 2011).

The community-based relational approach emphasizes relationships among parents and schools, focuses on the leadership development for both educators and parents, and bridges a gap in culture and power between parents and educators. Warren, Hong, Rubin, and Uy (2009) continue to stress the importance of valuing parents and promoting them to act as catalysts for change. One key lesson from the Warren, Hong, Rubin, and Uy (2009) study is that building authentic relationships is challenging but worthwhile. From their study, we learn that valuable opportunities are gained when school districts provide parents the chance to address their concerns.

Henderson and Mapp (2002) and Epstein (1995, 2011) are lead researchers in properly implementing parental engagement within schools. Including community members within American schools is not a far-fetched idea; it is just a foreign one. Schools have typically been exclusive agencies, only mingling with their own kind. Warren, Hong, Rubin, and Uy (2009) note that the perception of importance in 
incorporating community and parents is strong; however, the implementation is weak. Attempting a cultural change will prove to be beneficial, but at times frustrating. Blankenstein (2010) states, "Collaboration must take place with the overall success of the students in mind" (p. 147).

Identifying policy and framework. The only policy on parent involvement that mentions engagement is the Blueprint for Reform. It proposes to strengthen and support family engagement through specific programs. The suggested programs include districts and states allocating small percentages of Title I monies to monitor family engagement strategies encouraging a welcoming environment, open communication, and strong collaboration between families, teachers, schools, and districts (Department of Education, 2010a). The state program will create the Family Engagement and Responsibility Fund, a grant program to operate the best practices of family engagement. Unfortunately, the suggested programs give a goal, but provide no practical steps in attaining that goal. Engagement is more difficult than involvement, and these Blueprint for Reform programs still address engagement to be completed out of compliance. Engagement becomes systematic when improvement in student performance is realized (Weiss, Lopez, \& Rosenberg, 2010). This is why Epstein's framework for parent involvement is still utilized today. Used by most districts as the checklist to get parents involved, it is practical and appealing while making a strategic plan. Epstein's (1995) categories of parental involvement are:

Type 1: Parenting-Help all families establish home environments to support children as students.

Type 2: Communicating - Design effective forms of school-to-home and hometo-school communications about school programs. 
Type 3: Volunteering—Recruit and organize parent help and support.

Type 4: Learning at Home-Provide information and ideas to families about how to help students at home with homework and other curriculum-related activities, decisions, and planning.

Type 5: Decision Making-Include parents in school decisions, developing parent leaders and representatives.

Type 6: Collaborating with the Community-Identify and integrate resources and services from the community to strengthen school programs, family practices, and student learning and development. (p. 141)

During a quantitative study on high school parents and students, Catsambis (1998) noted that Type 1 had the most notable effect. Previous research from Horvat, Weininger, and Lareau (2003) noted that parents from middle-class communities contribute greatly in their children's schools because they possess the education and resources to give them confidence (p. 331). Supporting the conclusion from Gonzalez, Doan Holbein, and Quilter (2002), authoritative parental figures coming from EuropeanAmerican middle class parents were more involved than Asian-American and HispanicAmerican parents (Steinberg, Lamborn, Dornbusch, \& Darling, 1992, p. 1271). Both authoritative parenting and active involvement in a student's education have positive correlations to student academic success (Gonzalez, Doan Holbein, \& Quilter, 2002). Interestingly, contact between high schools and parents (Type 2) had a strong negative effect on course work completed during the student's senior year (Catsambis, 1998). Epstein persuades that communication is vital, but her model was created within the context of elementary schools. Communication, seen in the Catsambis (1998) study, was important in a high school setting. Therefore the strategies that must adapt from the elementary schools to high schools must include better communication with parents. If 
parents are more knowledgeable about the school and its operations, they can contribute to the ultimate success of their children (Smit \& Liebenberg, 2003). However, the tricky part is communicating to parents the value of involvement in schools as well as the appropriate method of communication; simply using an email will not work. "Parental involvement programs must include all families, even those who are not currently involved, not just the easiest to reach" (Epstein, 2005, p.179).

\section{Supporting Academics and Learning at Home}

The characteristics of high schools shape what schools are today, and that includes forms of parent engagement. High schools are more complex school systems than elementary schools. In high schools more students filter throughout the hallways during the school day, and more teachers are available to students due to the design of academic departments. These characteristics undermine parents' ability to remain effectively involved in their adolescent's education (Epstein \& Sanders, 2002); therefore the strategies used in elementary school are not sufficient in high school. Suggested high school strategies should embrace the "psychological distancing between parents and teens due to biological, cognitive, and psychological changes" (Suazo deCastro \& Catsambis, 2009, p. 93). Type 4, learning at home, was identified as a valuable concern according to Catsambis (1998) utilizing Epstein's framework (1995). Middle and high school parents believe that they cannot assist with more challenging high school subjects because adolescents are becoming autonomous (Eccles \& Harold, 1996). This concern continues to be a challenge and should be evaluated by high schools. Parents are a crucial sphere of influence needed for students to grow and learn accordingly to the overlapping spheres of influence model (Epstein, 1992). Targeting student achievement as the goal, school 
districts need to develop partnership activities to energize, motivate, and maintain positive influences.

Factoring parental influence. Dewey's thoughts on education set the tone for parental involvement in education. In his pedagogic creed, Dewey believed that school life should grow out of the home (Dewey, 1897). The family is the most important social system (Evans-Winters, personal communication, June 11, 2010). Previous studies regarding parent involvement within secondary schools include the effect on the family structure (Astone \& McLanahan, 1991), the expectations and encouragement for high school student success (Catsambis, 1998; Fan \& Chen, 2001), the credentials for children's success (Hoover-Dempsey, Bassler, \& Brissle, 1987), the parent's level of education and time spent with children (Cheng, Andrews, \& Yu, 2010), the desires of higher grades (Fehrmann, Keith, \& Reimers, 1987), and the desires of parents to have a caring relationship with the school (Heard, 2004). To go more in depth, Epstein (1992) states: "Students at all grade levels do better academic work and have more positive school attitudes, higher aspirations, and other positive behaviors if they have parents who are aware, knowledgeable, encouraging, and involved" (p. 1141). Yes, parents involved in education will make their student's life more productive; however, most research regarding parent engagement halts at the junior high level. Most researchers focus on elementary schools, since parent involvement is a necessity due to the age of the students. When students are old enough to have some independence, some mobility, and/or some other interests, parents and teens communicate less. Weaver's (2007) editorial presents themes of parent involvement in education. She argued that involving adults in education is important in developing good decision-making skills and "families have the potential 
to serve as a valuable contextual factor in the learning process" (Weaver, 2007, p. 6).

Taking a new angle to parent connection with schools, Steinberg, Lamborn, Dornbusch, and Darling (1992) use quantitative research methods to conclude that authoritative parenting does have a significant positive impact on adolescent school performance and engagement during the high school years. Authoritative parenting, defined by the combination of "high levels of parental responsiveness and high levels of demandingness" (Steinberg, Lamborn, Dornbusch, \& Darling, 1992, p. 1267), is more likely than other parenting styles to encourage academic excellence. Steinberg, Lamborn, Dornbusch, and Darling's (1992) theme is repeated by Epstein (1994, 2011), who confirms that when parents develop a positive attitude toward school, they will be motivated to get involved in their children's schooling (Epstein, 1994). Positive attitudes of parents will lead to positive attitudes amongst children (Epstein, 2011). In Fan and Chen's (2001) journal, Steinberg, Lamborn, Dornbusch, and Darling's (1992) understanding of the role of authoritative parents resurfaced.

Parental involvement, as represented by parents' supervision of children at home (e.g., home rules for watching TV, for doing school work, etc.), has the weakest relationship with students' academic achievement, whereas parents' aspiration and expectation for children's educational achievement appears to have the strongest relationship with students' academic achievement. (Fan \& Chen, 2001, p. 18)

Steinberg, Lamborn, Dornbusch, and Darling (1992) assert that understanding the role of authoritative parents will not only play a role in understanding a student's development through school, but it will also help educational practitioners and administrators design programs that encourage success in school along with a partnership with parents. Dealing with different types of parenting styles also affects different parental 
expectations based on racial/ethnic groups as well as socioeconomic factors (Yamamoto \& Holloway, 2010). For example, lower socioeconomic parents lack the sense of efficacy to help their children in school, especially in the higher grades (p. 200).

Respectful relationships among parents, teachers and students expand ownership for the educational experiences of children. “Teachers' expectations for children's academic achievement rise as they come to understand community concerns, including parents' interest in their children's education"' (Gold, Simon, \& Brown, 2005, p. 247). The result is curriculum and instruction that are more rigorous and culturally responsive. Redding, Langdon, Meyer, and Sheley (2004) account for the interconnections and multiple variables that are included in achieving student success in school. The most influential factors are family behavior, student mental capabilities, and the relationship between students, families, and school personnel. Family and school represent the primary variables influencing how a child grows up and develops both socially and cognitively. The significant link between home and school is the parents, since they contribute resources and encourage their children to grow academically. Parents' expressed goals, values, expectations, and aspirations for student educational attainments reflect the family that positively correlates to the student's well-being (Fan \& Chen, 2001; Hoover-Dempsey, Ice, \& Whitaker, 2009; Marchant, Paulson, \& Rothlisberg, 2001). A positive focus well-being leads to adolescent learning, goals for education, and achievement. More specifically, the student's attitude is wrapped in motivation and selfconfidence. Teens are more comparative. Their self-confidence declines, as does their motivation and engagement in school (Hill \& Chao, 2009). 
Standards-based education and accountability for learning have been the focus for high schools since the No Child Left Behind (NCLB) initiative. Unfortunately, the premise of NCLB failed. It used scare tactics to create an environment that did not result in high performance, in part due to disparities in socioeconomic status. Stiggins (2005b) deems success within standards-based education as igniting motivation for students in order for them to better perform. "The driving forces must be confidence, optimism, and persistence. All students must believe that they can succeed at learning if they try" (Stiggins, 2005b, p. 326). Reiterating that an intrinsic drive should always be the motivator to sustain learning, Pink (2009) disarms the carrot and stick mentality to motivate others. Extrinsic motivators typically squash any hope for motivation to come from within the person; it crushes creativity and encourages unethical behavior.

Motivation has been categorized by Maslow (1943) as a hierarchy of needs beginning with physiological and ending with the achievement of self-actualization. Self-actualization, determined within McGregor's Theory X and Theory Y, summarizes human needs of competence, autonomy, and relatedness by the self-determination theory (Pink, 2009). Today, the better form of motivation is identified as Type I (intrinsic) supporting three goals of autonomy, mastery, and purpose. Type I is powered by our innate need "to direct our own lives, to learn and create new things, and to do better by ourselves and our world" (Pink, 2009, p. 72). Motivation is involved in the reflective cycle between believing in one's self and taking personal responsibility for learning. Ames, Khoju, and Watkins' (1993) study found that children's perception of their parent's involvement was related to their academic self-competence, thus promoting the child's intrinsic motivation. Ginsburg and Bronstein (1993) were the first researchers, 
then later supported by Gonzalez, Doan Holbein, and Quilter (2002), to discover when parents react to grades with encouragement and praise, students were more likely to report characteristics of choosing more challenging tasks, displaying more curiosity, and increasing their overall interest to learn.

The model of parental involvement process by Hoover-Dempsey and Sandler (1995, cited in Hill \& Chao, 2009) summarizes the progress and process of actively getting parents involved in schools. At Level 1, the model suggests parents are motivated to become involved by a sense of efficacy for helping students succeed in school (Hoover-Dempsey, Ice, \& Whitaker, 2009). However, to address attempts at assessment reform, the process should no longer address the lower levels. Levels 1 through 3 are corrective methods of assessment of learning, whereas achieving Levels 4 and 5 address assessment for learning. Concentrating again on intrinsic motivation, the parent's involvement supports self-regulatory skills and instills those skills in order to support their own learning and achievement (Hoover-Dempsey, Ice, \& Whitaker, 2009; Jones \& Schneider, 2009). Promoting teenagers' ability to adjust long-term learning goals is one of the proximal learning outcomes known as self-assessment within the assessment for learning paradigm. Parents informed of the assessment for learning goals will help acknowledge the transparent expectations and requirements (Jones \& Schneider, 2009) of the program, thus attaining Level 5 of the Epstein model of the parental involvement process.

\section{Addressing the Assessment for Learning Paradigm with Parents}

When parents recognized and supported a school's emphasis on importance of effort, students positively recognized the message and were motivated to do well in 
school (Gonzalez-DeHass, Willems, \& Doan Holbein, 2005; Marchant, Paulson, \& Rothlisberg, 2001). Parents are not emphasizing mastery of standards (i.e., standardsbased learning), but instead parents stress the importance of learning. When that occurs, students feel more comfortable and capable of mastering academic work (Hill \& Chao, 2009). Research, to date, does not specifically mention how parent engagement will benefit assessment for learning; however, assessment for learning falls under the umbrella of what has been labeled as next generation learning. Next generation learning is personalized and tailored to individual learning needs as it prepares students for the acquisition of knowledge and skills while engaging students in their educational experience (Weiss, Lopez, \& Rosenberg, 2010, p. 4). The focus is on learning while working within the realm of standards-based reform. Teaching to the test is not desirable, but the challenge is how to effectively and efficiently manage those standards while inspiring lifelong learning. Research has documented that fostering the growth mindset in students will demonstrate mastery (Gonzalez-DeHass, Willems, \& Doan Holbein, 2005; Hoover-Dempsey, Ice, \& Whitaker, 2009). The assumption that needs to disappear is that school is the only place where and when children learn. Learning happens at home. Suggested activities from Weiss, Lopez, and Rosenberg (2010) support the role of learning at home as one of the four key roles that families can play in educational success.

Recommendations from past studies. Most parents can understand grades as the main source of communication since grades are the most used and expected (Guskey, 2006, p. 672). The purpose of grading is threefold including: product, process, and progress. Grades as a product are simply communicating where students are at a 
particular point. Grades as a process are the result of teachers taking a hodgepodge of grades along with effort and other extraneous variables such as attendance. Finally, using progress as a grading purpose means addressing the learning gains made from the beginning to the end of student learning. It will be difficult to combine the purposes of grading as product, process, and progress in the results of the assessment for learning success. Parents favor the upcoming assessment for learning practice because "it provides a more comprehensive profile of their child's performance in school (Guskey, 2006, p. 674). However, in the same breath, many parents see teaching practices that diverge from their own school experiences as an abandonment of academic rigor (Shepard \& Bliem, 1995). Shepard and Bliem's (1995) research focuses on examining parent opinions about standardized tests and to pushing back on the statistic that $76 \%$ of parents agree that students should have a cumulative exam before they graduate high school (Livingstone, Hart, \& Davie, 2001, as quoted in Mu \& Childs (2005)). Shepard and Bliem's study confirmed that parents find informal means of assessment more useful in gauging the success of their student. High school grades and student performance on state accountability tests will never be a good match (Guskey, 2006; Popham, 2007). Parents prefer assessments that make them think. For a few parents, assessments encourage competition (Mu \& Childs, 2005), albeit amidst the high levels of anxiety and nervousness from students (Barksdale-Ladd \& Thomas, 2000).

But ultimately, there is a huge difference between what parents know and what parents believe (Mu \& Childs, 2005). What parents know is the managerial stuff: what subjects are taken, why tests are given, where they can find information about tests, and who they can contact when they have questions (Barksdale-Ladd \& Thomas, 2000). 
What parents believe is more subjective and based on the needs of their students. It is possible to extend those published opinions of standardized testing toward assessment change. Any new assessment change or attempts at new form of assessments could likely be influenced by parent reactions. Parents can become a valid motivator within the school to help all students increase learning. Unfortunately, according to Mu and Childs (2005), parent attitudes towards assessment presented within the dynamics of high school are unknown.

Shepard (2000) constructed a paradigm to better understand assessment practices through curriculum, psychological, and assessment theories. Hoover-Dempsey, Walker, Sandler, Whetsel, Green, Wilkins, and Closson (2005) included student motivational, cognitive, and behavioral attributes since they are important to direct parent and teacher influences (p. 106). Educational leaders have the job to create opportunities for community confidence building and possibly become partners with parents to help transition new assessment practices in that opportunity. Ferlazzo and Hammond (2009) confirmed that parent engagement activities help to develop self-confidence in parents as they address educational concerns (p. 7). An administrator recognizing parent perceptions of assessment practices will strengthen the assessment initiatives at the school as well as cultivate a desire to learn from his/her students.

The question remains of how to engage parents in a role to prepare their children to understand educational reforms such as assessment. Hoover et al. (2005) found that parents feel alienated from school districts when changes occur without proper parent notification. They note, "If families have limited understanding of the educational system (i.e., the meaning of grades), any related information in appropriate formats can 
be helpful” (p. 127). Schools should continue to enhance parents' capacity of understanding the current state of high school curriculum. Cheng, Andrews, and Yu (2010) would wholeheartedly agree, since their quantitative research determined that the opportunity for parents to know about school-based assessment played the most important role from the variables analyzed in the study. The most effective school reforms are engaging parents in what is happening in the classroom, especially knowing what schools are doing to make sure all children are succeeding (Henderson, 2004). In addition, parents' perception about school-based assessments directly correlates and influences the students' perception as well (Cheng, Andrews, \& Yu, 2010).

Assessment reform, particularly the assessment for learning concept, has slowly crept in as an educational trend; however, parents are unaware of the assessment for learning presence. Ultimately, Hargreaves's words (2005) target the significance of this present dissertation study on parent perceptions of assessment in high schools. By understanding assessment, he wrote opportunities become available to "explore and interpret what conceptions of assessment for learning are held by different people" (p.

213). In the conclusion of the school-based assessment review from Hong Kong (Cheng, Andrews, \& Yu, 2010), it was suggested that this topic of perceptions merits further investigation due to the likeness of parent and student perceptions of assessment developed in their study along with the timing of the assessment for learning emergence in District Alpha.

\section{Chapter Summary}

Throughout the review of literature, the research emphasized conveys and intertwines the essentials of leadership of change, assessment practices past and present, 
and parent/family engagement. The educational leadership problem involving all three areas of interest is significant. There is a need to transition from parent involvement to parent engagement. The benefits spearheaded by Epstein's framework outweigh the challenges of change. The community has 'who-ness'. This means that regardless of positions, everyone has a valued opinion (Marsh, 2007, p. 113). There is a need to transition from teacher-led classrooms to student-initiated ones. The promotion of lifelong learning skills should be desired. Finally, the transition from top-down change to bottom-up change is inspiring. Embracing the idea that parents must be partners in the education of their children is critical for the success of standards-based learning. Tying the research to the context of District Alpha addresses an organizational problem for the district. District Alpha struggles to meet the internal and external issues of Assessment for Learning (A4L) and parent engagement while at the same time managing how to best lead through change. This dissertation research highlights a district's undertaking of a cultural change underlying an assessment initiative. The research, therefore, may discover criteria for determining the solution.

In sum, the review of literature suggests that the best leadership approach is distributed, the best change model is the Concerns-Based Adoption Model, and the best methodology is action research. Chapter III will articulate the design of the study to identify how a high school district can initiate a partnership between home and school in order to support high school students through assessment changes. This purpose supports the choice of action research as an ideal methodology for the study. 


\section{CHAPTER III}

\section{METHODOLOGY}

Taking your ideas and passions and translating them into a realistic and doable project. (Butin, 2010, p. 15)

Chapter II presented academic research framing the significance of initiating change in high school assessment with the collaboration of parents. In recognizing the challenge of trying something new, a qualitative research approach, specifically action research, was chosen for this study. Action research synergistically intertwines theory and practice. The study's ebb and flow centered around the deficiencies in parents' understanding of Assessment for Learning (A4L) and in parent engagement in high school academics, as well as how to overcome those deficiencies. Collecting and documenting the attitudes, opinions, and concerns of families who have children involved in the school district's implementation of Assessment for Learning (A4L) was the study's goal.

As reported in Chapter I, District Alpha's first steps in the A4L process began in 2007 with the professional development of the teachers. The professional development highlighted Marzano's (2007) instructional strategies for effective student learning, which include learning targets, emphasis on vocabulary, frequent feedback, and opportunities of student self-assessment. After 7 years, the staff has developed the skills to communicate assessment reform with students and is eager for the reform to become more encompassing. I saw a necessity for District Alpha to include parents in the efforts 
toward Assessment for Learning (A4L), thus improving the organization's effectiveness regarding its implementation of Assessment for Learning (A4L). Action research is the best method for an educational leader seeking answers to bringing about the change. This chapter will share why action research was the chosen methodology as well as elaborate about the particular action research theoretical lens: Living Educational Theory.

\section{Characteristics of Action Research}

Dewey (1916) wrote how experience and intelligent actions were linked in a

cycle. Describing the process as the scientific method, Dewey reflects:

First is the genuine situation of experience; secondly that a genuine problem develop within this situation as a stimulus to thought; third, the information and the observation needed to deal with it; fourth, suggested solutions occur to him which he shall be responsible for developing in an orderly way; fifth, the opportunity and occasion to test his ideas by application, to make their meaning clear, and to discover for himself their validity. (p. 192)

The positivist view of research came forth with a methodology focusing on proposed ideas as testable and, if designed well, the results are reproducible. However, there are deficiencies in positivist science for gaining knowledge for use in solving problems members of organizations face (Susman \& Evered, 1978). Action research is a method used to generate and conduct action as a means for exploration. Education is a way to uncover truth. Too often we see research that wants us to accept the author's version of truth. Action research begins where there is value in the research for both the researcher and others. The purpose is to create change and address a need. In the end, the dissemination of the results will be supported and well documented (MacLean \& Mohr, 1999).

The term action research was first introduced by Lewin in 1946 to address a specific problem within a specific setting, while maintaining an interest in improving 
quality and creating social change (Cochran-Smith \& Lytle, 1993; Merriam, 2009). Action research became an established methodology since its methods were future oriented, collaborative, agnostic, and situational, while its success rests on understanding the value of how a problem is solved and appreciated during the process (Susman \& Evered, 1978).

Unique to action research is the data interpretation as a conglomeration of multiple views. Herr and Anderson (2005) state, "Collaboration can be a crucial component of action research which not only might have a greater impact on the setting, but can also be more democratic" (p. 36). Action research allows for equal participation, and this is a highlight of the study. In order for action research to be effective research, it is important to acknowledge the influence of the problem, embrace the opportunity to change it (MacLean \& Mohr, 1999), and the capability of generating a valid explanation of our educational influences (Whitehead, 2010). Assessment practices have been scrutinized since Black and Wiliam's (1998) initial study. Parents have traditionally withdrawn as support systems for high schools and at that level only communicate with schools when discussing grades. This dissertation research ultimately desired to try something new by involving parents to find better success for their children in school, inspire a desire for life-long learning, and find sustainability for Assessment for Learning (A4L).

The role of the researcher is pivotal to the collaboration sought in action research. In action research, the researcher is actively involved, possibly as the instrument, collecting data through observations, document analysis, or interviews. The researcher is an insider who possesses local knowledge about the setting, something that is difficult for 
other academic researchers (Anderson, Herr, \& Nihlen, 2007). Action research is inquiry that is done by one with insiders of an organization or community, but never to or on them (Herr \& Anderson, 2005). Researchers want to collect the data at the site where participants experience the situation. As stated in Friend and Cook (1996), "In collaboration, listening is especially crucial. Listening is a primary means for gaining information, but it is also a means of conveying interest in the message of others" ( $p$. 137). Heeding these words, this action research used communication to build the story of implementing Assessment for Learning (A4L) in the high school district. To best describe the experience of the setting is to utilize multiple sources of data. Action research embraces the variety of sources since the tendency of the research is cyclical, allowing for multiple sources to tell the themes or patterns necessary to help make sense of it all.

Action research requires that the researcher identify a problem, respond to the problem with a plan, implement the plan, collect data, reflect and analyze, and then repeat the cycle again (Kemmis \& McTaggart, 1988, 2008; Glanz, 2003). The repetitive cycle stresses action completed during research to be reflective, insightful, and flexible.

Kemmis and McTaggart (1988) state,

The plan is constructed action and by definition must be prospective to action-it must be forward looking. It must recognize that all social action is to some degree unpredictable and therefore somewhat risky. The general plan must be flexible enough to adapt to unforeseen effects and previously unrecognized constraints. (p. 11)

The Kemmis and McTaggart (1988) design highlights the cyclic pattern similar to the steps described as the scientific method presented by Dewey. The stages overlap to allow the process to be open and responsive. Being open and responsive to the feedback 
received from the sources of data allows a particular viewpoint as a theoretical lens to review the issue while defining local and public knowledge (Cochran-Smith \& Lytle, 1993). Returning to the premise of action research, the researcher's role is influential and the influence lies in the researcher's theoretical lenses. The two theoretical perspectives supporting the study are a form of action research called Living Educational Theory developed by Whitehead (2008), and the Concerns-Based Adoption Model (CBAM) developed by Hall and Hord (2010). Whereas Living Educational Theory was important in conceptualizing the study, the Concerns-Based Adoption Model was important to the implementation of the research processes.

\section{The Living Educational Theory}

While 'walking the walk and talking the talk' of Assessment for Learning (A4L), I have noticed a lack of support from parents. As a teacher researcher, I have begun to question why and hypothesize how to improve my teaching and student learning. Whitehead (2008) summarizes that the questions we ask about our teaching profession can be influential in what we do to convey learning to our students, thus creating a living educational theory. As explained by Whitehead (2008), a living educational theory emerges to best explain how teacher researchers are inspired by the question "how do I improve what I am doing?” A summary from Whitehead (1989) best explains this theoretical framework.

In a living approach to educational theory, action researchers present their claims to know how and why they are attempting to overcome practical educational problems in this form:

- I experience a problem when some of my educational values are negated in my practice.

- I imagine a solution to my problem.

- I act in the direction of my solution. 
- I evaluate the outcomes of my actions.

- I modify my problems, ideas, and actions in the light of my evaluation. (p. 98)

Virtually an unknown approach to research in the United States, the living educational theory approach to action research evolved in England, particularly at the University of Bath by Whitehead $(1989,2008)$. The evolution of this theory is, in part, a reaction to the notion that the disciplines of education (philosophy, psychology, sociology and history of education) could explain the educational influences of individuals in their own and in each other's learning (Whitehead, 1989, 2008). Instead, Whitehead argues that educational influences and values of freedom, compassion, and respect grounded in emotion (both professional and personal) provide the best foundation to produce an explanation for educational influence in the learning of others (Whitehead, 2008). My thoughts and observations are grounded as personal, emotional, and reflective. I designed a study that demonstrates leadership, creates partnerships, and develops future opportunities for parents to remain supporters of academics in the high school.

Even though I am the science department chair, I also teach three classes. I am exposed to the same professional development as everyone else. I apply the professional development goals to a classroom setting, and I understand the frustrations of my department. Other teachers note the lack of parental support and join me in hypothesizing that, if parents became more involved, A4L would prosper. I believe the lack of support is due to parents being unaware of the true potential of the program and only looking at it as a new grading policy. Even though this lack of support presents a challenge, I feel including parents is District Alpha's next step in full implementation of its assessment vision. 
I am also more comfortable with the living educational theory's methodology. Emphasis placed on living theory allows my study to be a "vehicle for predictions and explanations" (Whitehead, 1998, p. 3). Relating back to creating a hypothesis in science experiments, the if/then statement best suits the science teacher within me but also allows me to attempt to increase the organization's effectiveness in implementing Assessment for Learning (A4L). Just like formative assessment promotes students to reflect—such as Where am I going? Where am I now? and How can I close the gap? (Chappuis, 2005)— those same questions can direct the methodology of action research under the living educational theory. Instead of taking a general approach toward education, the living educational theory "resonates with your own life-affirming energy, values, and understandings" (Whitehead, 2008, p. 118).

The living theory processes resemble the action research reflection cycle. The planning section imagines the solution to the educational problem. The teacher researcher acts in the direction to follow the plan, evaluates the outcomes, and modifies the problem. It is the problem-forming and solving that Whitehead $(1989,2008)$ strongly affirms that will accurately explain teaching and learning in his classroom and therefore in mine. Conducting this research is a personal and professional investment that will benefit District Alpha's future.

\section{Research Purpose and Questions}

"Good qualitative research has value to the students, to the researcher, and to others in the educational community" (MacLean \& Mohr, 1999, p. 166). Black and Wiliam (1998) established the need for assessment reform since there were discrepancies between the intention of good education and the reality of it. Ultimately, students were 
not retaining knowledge and were unable to apply concepts later on. Wiggins (1998), Marzano (2003, 2007), and Stiggins (2005a, 2005b, 2007) have modeled the benefits of Assessment for Learning (A4L) strategies. However, just as Prestine and McGreal (1997), Carless (2005), and Boyle and Charles (2010) have highlighted, schools have difficulties with sustaining strategies long term. Despite the amount of research conducted on positive influences of parent's engagement in school, little is known about how parents help implement change in assessments, specifically in the high school. With little or no direction about how to best work through the problem, I had a curiosity about how to properly implement new assessment initiatives while engaging parents in the process.

To reiterate, the purpose of the study, as stated in Chapter I, was identifying how a high school district can initiate a partnership between home and school in order to support high school students through Assessment for Learning (A4L) changes. More specifically, this action research study was crafted to answer the following questions:

1. What attracts parents to supporting Assessment for Learning (A4L) reforms at the high school level?

2. How can parents value assessment reform and comprehend the goals of Assessment for Learning (A4L)?

3. What affects parent perceptions and influences their support of the district's Assessment for Learning (A4L) initiative?

4. How can educational leaders sustain home/school collaboration during the Assessment for Learning (A4L) reform process? 
This study examined the topic of assessment reform practices at the high school level focused on parent/family support through the following steps: parental information nights, surveys, and a focus group. The study:

- Introduced the concepts of Assessment for Learning (A4L).

- Reported upon study habits at home and in school.

- Uncovered the opinions and perceptions of parents regarding the objectives of the A4L.

- Offered a solution to how parents can become an A4L support.

- Adjusted reform strategies after the focus group to include teachers.

\section{Data Collection and Procedures}

Prompting this dissertation study for me was an Asset program survey during May of 2010. District Alpha promotes an asset program that highlights values to encourage young adults to be respectful, considerate, and accountable for their actions. Typically, the student responses direct the program toward particular discussions and presentations conducted monthly in advisory periods.

Fueling further investigation were the perceptions gathered from parent participants in the 2011 pilot study I conducted. By inviting parents to become more involved and be able to express their ideas, the parents felt valued. Their candid responses indicated they were in the initial stages of understanding formative assessment. Through their evaluations of learning verbs, grades, and self-assessment, it was confirmed that taking time to discuss matters is just the beginning of finding ways to encourage student achievement. Going beyond what was reported, there is a need to encourage change within the dynamics of a high school and its parents. Webb and Jones 
(2009) describe the change as a cultural one and Stiggins (2005b) highlights motivation to aid in change. "The driving forces must be confidence, optimism, and persistence. All students must believe that they can succeed at learning, if they try" (Stiggins, 2005b, p. 326). It was apparent from the responses gathered, from three parents volunteering for the pilot study, that parents value their relationships with their children. This action research dissertation then became the more responsive and collaborative study with parents.

The data interpretation for this action research incorporated more opportunities for information and discussion than the initial pilot study. The variety of data collection included an informal presentation of the Assessment for Learning (A4L) objectives (particularly student self- assessment), surveys of parent perceptions regarding those objectives, a focus group to actively listen to a group of parents, a review of student feedback in forms of surveys (Asset program and 5Essentials) and finally self-assessment documents. I had approval from District Alpha's former Assistant Superintendent for Curriculum, the Director of Instruction, and the building principal to proceed with the research.

\section{Setting and Participants}

District Alpha and the community have a symbiotic relationship. Both the high school district and community maintain high values of finances, discipline, and academic rigor. Based on the 2010 American Community Survey provided by the United States Census Bureau, the characteristics of the community are primarily single family homes with steady incomes and parents who have had some college courses with about half of the population obtaining a college degree. The trend of parent involvement, as defined 
by Illinois State Board of Education, remains consistent between District Alpha and the elementary district. The involvement of the parents in the junior high is average for the state, while participation amongst other high schools is above average.

The volunteering participants in this study were parents who had a child(ren) attending one of the two high schools associated with the neighboring elementary district. Due to their proximity to the schools and investment in the district, the participants were a convenience sampling. Those who participated in the informal presentation surveys and in the focus group were parents of freshmen, since the annual freshmen final review night in December 2012 was used as the venue to begin discussing A4L with families.

\section{Informal Presentation}

District Alpha invited freshmen parents of its West and Central schools to an informational presentation on December 3 and 4, 2012 via flyers (see Appendix C) sent home, email blasts, and information posted on the district's website. The purpose of this presentation was to discuss the significance of final exams and how to best prepare for the exams. In preparation for the first time that the freshmen would take final exams, the presentation was also beneficial for parents to comprehend the challenge that lay ahead. Parents had the choice to attend the informal presentation. Attendance for the two nights was close to 500 people, including both students and family members.

During the evening's presentations, each academic department from the high school presented their semester objectives and emphasized key study skills for their final exam. As discussed with the Director of Instruction, I introduced the definitions of formative and summative assessment, using the final exam as an example of the latter. I elaborated upon student self-assessment as it pertains to the Assessment for Learning 
(A4L) program and is best suited for final exam review. The bridge between summative and formative assessments in high school is preparing for final exams through examining what you know, spending time on what you do not, and continuously evaluating and reassessing learning. At the end of my presentation, I requested parents in the audience to complete a survey that was included in the evening's paperwork. Thirty-seven responses from the West school in addition to the 68 at the Central school provided the study with 105 responses gauging opinions on the night's presentation.

\section{Surveys}

Surveys were a viable option for obtaining perceptions and opinions of those participants, as they provided an avenue to collect information from a large sampling and complete the task anonymously. The dissertation study utilized several survey responses including in-district, state sanctioned, and personal surveys given specifically for use in this dissertation study.

2010 Asset program survey. The entire student body at District Alpha's Central school (approximately 1,900 students) answered a 40-question pen and paper survey categorized into areas of support, empowerment, expectations, and constructive use of time. The survey used a 5-point Likert scale with students choosing the option that best matched their opinion. The survey was conducted during a student's 25-minute advisory period within the school day. The asset program director administered the survey within a 2-week time period. Results from this survey, included in the following chapter, drove the initial 2011 pilot study as well as a question for the focus group discussion. The inhouse district survey is in Appendix A. 
Informal presentation survey. As previously mentioned, a total of 105 parents completed the paper-and-pen survey included in the night's paperwork available as participants walked into the auditorium. The survey used the 5-point Likert scale for five questions to address opinions of reform and the goals of the A4L objectives. The survey appears in Appendix A. The survey provided immediate feedback and produced a high return rate as the parents completed and dropped off the survey into covered boxes before they left the auditorium those particular nights. Space reserved on this survey for parents to include their contact information (name and email address) in order to be invited to participate in a focus group at a later date. Any participation in this study beyond the informal presentation was solely based on volunteers and consent of the participants.

The 5Essentials survey. The state sanctioned survey given for the first time during the spring of 2013 offered a comprehensive assessment of a school's organizational culture and school effectiveness using the indicators of: effective leaders, collaborative teachers, involved families, supportive environments, and ambitious instruction (Illinois 5Essential, 2013). Under legislation (Senate Bill 7, PERA), the State Board now mandates, on a biennial basis, a learning conditions survey from teachers, students, and parents using a Likert-like format. Individual participation in the Illinois 5Essentials survey is completely voluntary. A reported 5Essentials summary is only comprised of the teacher and student survey components, with the parent portion only to be reported to school districts. To receive a report for your school/school district, at least $30 \%$ or more of parents must have responded (based on the total number of students at the school), and $50 \%$ of both the teaching/instructional staff and student body participated. UChicago Impact administered the survey on behalf of the Illinois State 
Board of Education. For District Alpha's Central School, 55.2\% of its students, $61.5 \%$ of its staff, and less than $10 \%$ of parents took the 30-minute online survey on a secure website in 2013. Survey results for the 2014 5Essential survey incorporated only a $26 \%$ student response rate, $67 \%$ teacher response rate, and $11 \%$ parent response rate. The survey questions for students and teachers are included in Appendix A.

Focus group survey. Conducted at the end of the focus group session, the penand-paper survey designed for this research was passed out to the participants at the end of the roundtable discussion. The survey used the 5-point Likert scale for seven questions to measure the final views of the parents reflecting upon the discussion of the focus group and to indicate the future implementation of A4L. All seven participants completed the survey, and it was dropped off into a covered box in the adjoining room as they left. The survey appears in Appendix A.

\section{Focus Group Interview}

Interviewing offers an opportunity to shed light on the research and to probe for underlying principles (MacLean \& Mohr, 1999). The purpose of the focus group interview was to receive high quality data and in-depth perspectives in a social situation where the parents were able to consider their own views in the context of the views of the others (Patton, 1987). For this particular focus group, the small group of parents sat in a round table format and responded to questions addressing the objectives of A4L. The researcher facilitated the discussions of summative versus formative assessment practices, desired study skills (i.e., self-assessment), and ways to support learning at both home and school. The group of seven participants met in the library classroom of District Alpha's Central school. The group only met once in February 2013 for approximately 90 
minutes. The focus group format used a semi-structured interview style integrating both open-ended and follow-up questions. Good questions reflected familiar language and leading questions were avoided in order to allow for emergence of the true lived experience of the participants without the researcher's bias overshadowing the data. The semi-structured focus group protocol is included as Appendix B. Interviewees were not pressured to answer all the questions, thus establishing a trusting rapport with the respondent (Patton, 2002).

Participants indicated their interest in participating in the focus group on the informal presentation survey. It was my intent to limit coercion into volunteering for the focus group by using an email recruitment format. From the 10 contacts voluntary provided, only 7 responded favorably to the focus group invite and the others were not contacted again. The seven who responded knew the research requirements as stated in the recruitment letter and made their decision to participate based upon those research requirements. These requirements were also reiterated in the consent form for audiotaping of the focus group before the focus group discussion began. Both the recruitment letter and consent form are found in Appendix C. The focus group was transcribed a month later.

\section{Document Analysis}

Final source of data for interpretation was the document analysis of student selfassessment forms. Self-assessment documents are utilized by the entire science department at the Central school in some form or another. The science teachers are encouraged to use a format of self-assessment before an exam, after an exam, or after the completion of a school year quarter or semester. I requested the biology teachers of my 
department to provide copies of any self-assessment form given during the first semester of the 2013/2014 school year, excluding the student's name. Avoiding using my position as the department chair to influence the collection of these documents, I received only 71 forms from my initial and only request of my department.

Even though the format differed slightly among teachers, the targeted questions involved evaluating student academic effort, suggesting ways to improve grades, and proposing ways for the student's family to become involved. An example of the selfassessment document is available as Appendix D. The open-ended format of the document provided more of an opportunity for students to express their thoughts, and provided me with more of an opportunity to peek into freshmen's analysis and interpretive skills.

\section{Study Timeline}

September-November 2011: Reviewed the 2010 Asset Program Survey and concluded Pilot study.

December 2012: Freshmen final review night presentations. Parents participated in the survey and provided contact information for focus group.

January 2013: Sent email invitations to attend the focus group to parents who volunteered their information.

February 2013: Hosted focus group and encouraged active participation in order to get specific feedback regarding final exams, study habits, and began to brainstorm solutions for A4L sustainability.

November 2013: Reviewed published 5Essentials survey results. 
December 2013: Collected and reviewed the self-assessment documents collected from teachers in the science department.

\section{Data Analysis and Procedure}

There is a personal side to change, and this study placed change in high school assessment and decision making under the microscope. Feelings and perceptions have been identified as concerns (Fullan, 1969, cited in Hall \& Hord, 2010) and Hall and Hord developed a model to incorporate the experience of change through the stages of concerns (SoC) labeled as unrelated, self, task, and impact. This approach is consistent with action research as, “Typically, we do not get a plan absolutely 'right' and in fact, as we implement a plan, the very implementation raises new issues or things we hadn't expected or anticipated" (Anderson, Herr, \& Nihlen, 2007, p. 146).

Hall and Hord (2010) developed the Concerns-Based Adoption Model (CBAM) to help identify stages in a change process as well as to implement goals of social change. Through periodic check points, the CBAM helps determine if all parties involved have consensus, thus moving toward the goal to improve student achievement in a safe and orderly environment (Loughridge \& Tarantino, 2005). Using the CBAM model and its anticipating patterns, it is possible to facilitate change using action research as a foundation. The Kemmis and McTaggart (1988) model, introduced in Chapter I, represents a spiral repetitive process for conducting research and analyzing data. The 'plan-act \& observe-reflect' flow works well with the overlay of CBAM checkpoints.

The idea is for engagement of the participants to adjust the pace and rigor of the study as together they moved through the seven stages of concern. 


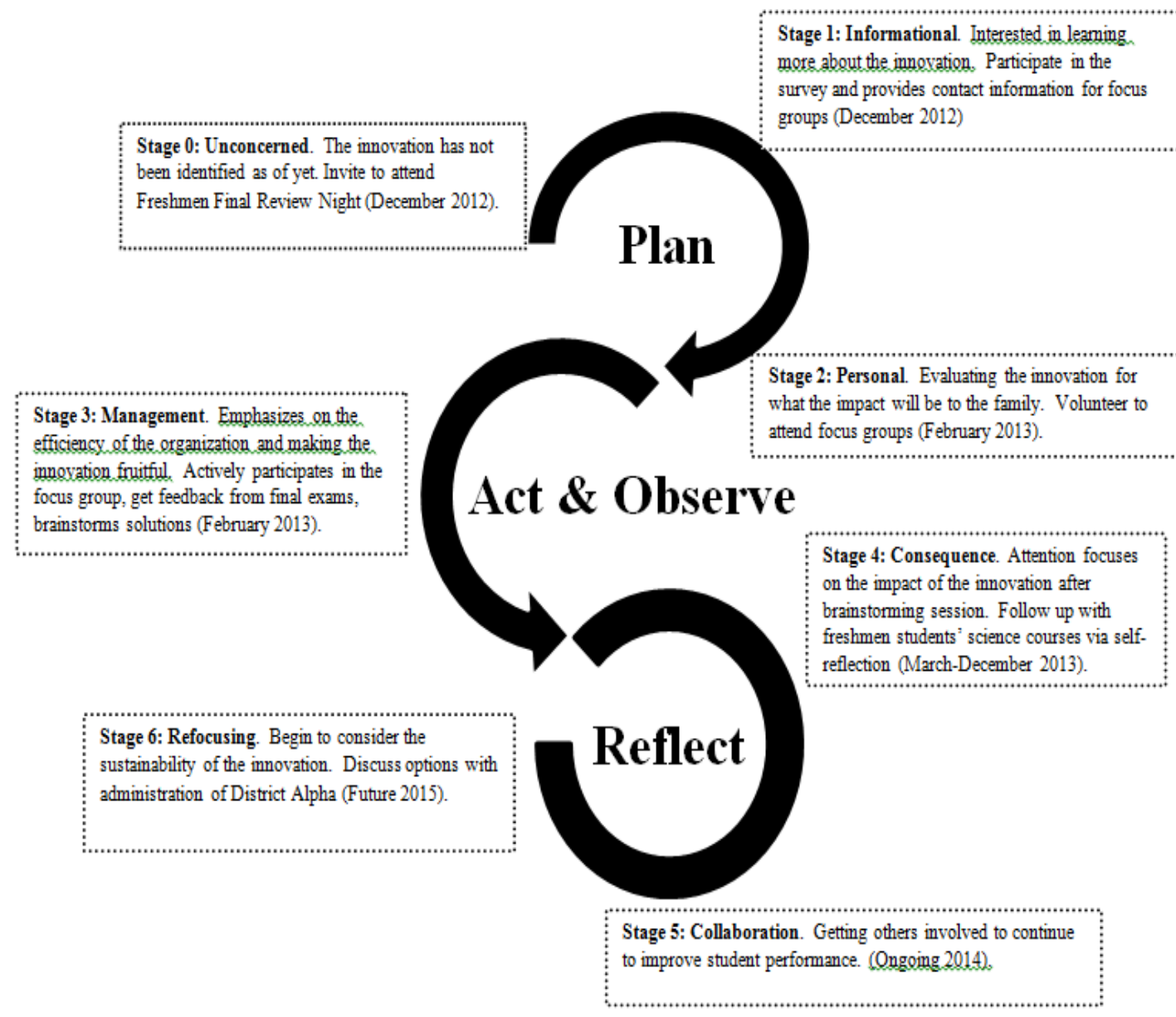

Figure 2. Kemmis and McTaggart (1988) Model with Overlay of the CBM's SoC

Since parents are not identified as change agents for District Alpha, this study began at the Unconcerned level (Stage 0). Parents may not be interested in assessment practices and may only be concerned with their child earning a particular grade as seen in the pilot study; hence, why they attended the night to learn how to successfully manage their child's freshmen year exams. By the end of that night, parents moved to SoC's Informational level (Stage 1) and their responses to the brief survey indicated what information struck a chord with parents and what questions remain unanswered. The 
next stage developed over the next several months. Parents may have noticed a change in their child's study habits, thus the change became more personal. The Personal level (Stage 2) is where parents began to evaluate the innovation, i.e., A4L, and how it impacts their family. The parents began to formulate questions, concerns, or comments on how the change could be sustained. At this time, the seven parents responded positively to the invitation to participate in the focus group.

The sense of collaboration between the school district and home began to blossom at the Management level (Stage 3) of CBAM. The focus group interview made the innovation fruitful. Parents actively participated in the focus group, as I actively listened and facilitated, thus creating meaningful data. With the learning from the focus group in hand, I made a transition to the impact of A4L both at home and at school, the Consequence level (Stage 4). The document analysis review supported the emerging patterns from the focus group analysis as well as provided evidence of $\mathrm{A} 4 \mathrm{~L}$ in the classroom. The last stages of concern (Collaboration-Stage 5, and Refocusing - Stage 6) are about the impact of further collaboration and future refocusing. If the parents from the focus group were pleased with the academic results of their child, then hopefully, the good news about A4L will be passed along. District Alpha may implement the strategies presented in the study and build from there for possible professional development opportunities.

Using CBAM's SoC as a tool to analyze data is beneficial to better understand how a partnership between family and the school can mature. It is the themes that emerge from the data that sustain the partnership and give it longevity. Dilley (2000) suggests that the researcher look consciously for "patterns in conversations" (p. 2), an 
approach that is supported by a coding strategy to identify themes hidden within the data that was developed by Strauss and Corbin (1998). Greeno, Collins, and Resnick's (1996) research regarding the different approaches toward issues of cognitive learning provided a way to consolidate CBAM's seven stages of concern into three themes that were more relatable to the parents volunteering for the focus group. I felt the themes of knowing, learning and transfer, and motivation and engagement helped to focus how phenomena might possibly be related to one another. The themes were a useful approach for organizing the patterns in the focus group transcript.

The procedure used to unveil the data themes began with reading the transcriptions of the focus group once in full. Following that read, I used three colors, each presenting the themes from Greeno, Collins, and Resnick (1996), to highlight text that best related to those areas. I cut and pasted those sections that were similar into a document to better target the specific themes of research discussed by the parents. Mulling over the text did generate buckets as Marshall and Rossman (2006) suggest be used to compare and relate data back to the research questions. I continued to use these buckets as I reviewed the survey questions, the corresponding responses, and the student documents. Combining all sources of data allowed insights and significant themes to be applied as possible answers to the research questions. This iterative process made it possible to draw significant conclusions regarding building parent support for A4L.

\section{Quality of the Study}

The techniques mentioned to complete this study produced data that were reasonable, well-explained, credible, transferable and dependable (Merriam, 2009). A good study is conducted ethically and the data says what you need it to say, thus 
displaying trustworthiness. Reliability complements trustworthiness as it provides enough information for a reader to be able to make reasonable comparisons to other situations (MacLean \& Mohr, 1999) and how similar effort would work to summarize and be applicable to other situations (Cochran-Smith \& Lytle, 1993). It is important to mention reliability since, in social situations, human behavior is never static. Using the CBAM model's Stages of Concern encouraged reliability since change will likely only occur after each stage of concern is addressed. The information, such as patterns in responses from the survey and focus group, uncovered at each step drove the decisions to identify the challenge of a high school district initiating a partnership between home and school in order to support high school students through assessment changes.

Even though the study began with a pilot study of three interviews and expanded to include seven parents of the District Alpha, transferability is evident. However, it is impossible to generalize the data received from one high school district in Illinois. The lack of generalizability has been used to discount the value of action research (CochranSmith \& Lytle, 1993). A way to stress transferability is the method of triangulating data to establish internal validity for the study by using multiple sources of data (meeting, survey, focus group, and document analysis). One way the researcher encouraged validity was displaying reflexivity to the participants by explaining her position within the district, the reason why she was involved, and her foresight of the need for more collaborative relationships with parents in secondary schools.

\section{Ethical Considerations}

Anticipated risks and potential benefits are the final considerations for the study. Since action research is considered backyard research (Creswell, 2009), there needs to be 
an established standard of objectivity that includes clarity and replicability (Bryant, 2004). The Institutional Review Board (IRB) addressed the protection of the individuals who volunteered for the study and their approval of two protocols minimized conflicts within the study (Butin, 2010). The protocols, approved in November 2012 and in November 2013, were developed under the guidance of Dr. Linda Lyman of Illinois State University. Interviews were conducted at District Alpha's Central school providing privacy and confidentiality to each participant as well as providing comfort to them. In the discussion portion of the results that follows, there are no differentiation or identifiable characteristics between the parents' quotes.

For the researcher-participant relationship, participants knew the researcher's relationship to the topic, and it was presented within the study's limitations. The researcher created an environment where all voices were heard and the participants' trust in the study produced results. MacLean and Mohr (1999) describe this as responsiveness, a good term to describe the kind of ethical behavior you strive for as a teacher researcher. Ultimately, as a researcher, I treated the study and participants with respect, disclosed my plans, methods, and results, and acknowledged their beliefs to remain ethical during research (Cochran-Smith \& Lytle, 1993).

\section{Chapter Summary}

Qualitative research has made gains in the past few years but most schools are still bound to quantitative studies (MacLean \& Mohr, 1999). School administrators fail to make use of the qualitative data to help address gaps in social situations. "Only educational research that fully represents school realities can provide a sound basis for the valid and reliable assessment of the work of schools" (MacLean \& Mohr, 1999, p. 
123). Action research requires infiltration from the researcher into the study, while at the same time allowing for a normal routine. Action research focuses on an educational problem that can be solved through a variety of ways locally and with help from participants. The best approach for facing the organizational problem of how to best engage parents within the Assessment for Learning (A4L) paradigm was living educational theory.

The surveys included in the study provided an opportunity for students and teachers to have a voice in improving their schools. Their results were used to identify resources and efforts needed to find sustainability in an assessment reform. The summary provided from the 2013 5Essentials identified that District Alpha's Central school is well organized for improvement (Lincoln-Way Central 5Essentials, 2013). Through the opinions of the teachers and students, it displayed the crucial role that feedback aids in school reform. What they shared about their school predicted whether the school is likely to improve.

The key component of the methodology was the focus group interview that was restricted by the small sampling of parents who volunteered. However, just listening to parents' nuggets of wisdom was the benefit that Friend and Cook (1996) identified. From the interpretation of the focus group's transcription, themes emerged in the relation to the four research questions and these will be discussed in the following chapter. 
CHAPTER IV

FINDINGS AND DISCUSSION

Discovery consists of looking at the same thing as everyone else and thinking something different.-Albert Szent-Györgyi

This chapter examines the research questions that drove this action research study. "Action research produces knowledge grounded in local realities" (Herr \& Anderson, 2005, p. 96). I sought to improve the formative assessment being implemented at District Alpha by reaching out to its families. Identifying factors pertaining to continued parent support for implementation of Assessment for Learning (A4L) at the high school level has been a process of summary and interpretation of various data sources including surveys, a focus group interview, and analysis of documents. The purpose of this chapter is to report the results and analysis of that data.

Crucial data came from the focus group volunteers whose perceptions and opinions were heard in an open-ended subjective manner. Other parents had the opportunity to voice their opinions on both state sanctioned and personal surveys. A student-generated data component was also included using survey responses to both state sanctioned and in-district surveys, as well as self-assessments from their freshmen science course. Analyzing all data sets generated three cross-cutting themes to answer the first three research questions proposed. These interlocking and overarching major themes of Effort, Closing Gaps, and the underlying theme of Fair Ranking emphasize 
how the attractive, valued, and influential features of A4L can be developed to build support from families.

\section{Action Research Purpose}

Anything worth doing is worth doing slow.-Mae West

The purpose of this action research study was to identify how a high school district can initiate a partnership between home and school in order to support high school students through Assessment for Learning (A4L) changes. The researcher examined the possibility of building parent support for the continued implementation of formative assessment through an informal presentation, a focus group, surveys, and document analysis. Constructs from the Stages of Concern (SoC) in the Concerns Based Adoption Model (CBAM), developed by Hall and Hord (2010), supported the progress of parent perceptions toward a home/school partnership regarding assessment. Additionally, this study created a living educational theory (Whitehead, 2008) through action research that sought to build collaboration with parents to support implementation of Assessment for Learning (A4L) at a suburban high school district south of Chicago.

Living educational theory is apparent every day that I am at work within a high school science department. Since 2007, District Alpha has made strides in adopting portions of formative assessment each year. This transformation of assessment practices has been crucial in making a more independent, self-reliant student graduating from District Alpha. I have imagined the solution to sustain formative assessment at District Alpha, and believe that it lies in the collaborative involvement with parents. Collecting data for the study began December 2012 with the informal presentation, then progressed through 2013 with conducting surveys, review of self-assessment documents, and a focus 
group interview. Concerns-Based Adoption Model's stages of concerns (CBAM's SoC)

were the foundation for the methodology and were used to address the degree of parental support for A4L. The stages of concern pertaining to the research are identified through the narration of the data while addressing the feelings, perceptions, and concerns of families regarding assessment reform.

The timing of this study is significant as it is the story behind the numbers published in the state's new school report card emphasizing the 5Essentials that define a quality school. Its data was collected simultaneously as the focus group met. However, as action research allows for more narration of participant responses instead of strictly focusing on numbers, its feedback is more valuable. For example, District Alpha on the survey's Involved Family category (one of the five pillars of the report) was acknowledged as 'strong,' However, this rank is an average between a 'very strong' rating for School Resources and Parent-Teacher Trust, and a 'neutral' on the Outreach to Parents and Parent Involvement categories for the school. It is the meaning of the ambiguity of the 'neutral' rank that this study hopefully clarifies in terms of parent support being existent or non-existent.

\section{Research Questions}

Since there are a limited number of studies addressing A4L at the secondary level, most administrators have little idea about how to start the process of implementing assessment for learning within their schools. It is a delicate balance between curriculum, instruction, and assessment. District Alpha has been grounded in the eight learning strategies during its transformation period toward A4L. Teachers are aware of and evaluated by these goals; students have been exposed to these goals, but what do parents 
understand? The results and analysis shared in this chapter provide a start to build parent support for $\mathrm{A} 4 \mathrm{~L}$ as there is no district policy for family engagement. As stated in Prestine and McGreal (1997), restructuring initiatives must be radical in order to complete a change toward how things should be. It is imperative that parents are engaged and supportive of their students' and the school's academic successes.

The research questions stated for this study were:

1. What attracts parents to supporting Assessment for Learning (A4L) reforms at the high school level?

2. How can parents value assessment reform and comprehend the goals of Assessment for Learning (A4L)?

3. What affects parent perceptions and influences their support of the district's Assessment for Learning (A4L) initiative?

4. How can educational leaders sustain home/school collaboration during the Assessment for Learning (A4L) reform process?

The questions were primarily addressed by parent responses during a focus group interview. Opinions and perceptions of parents regarding the objectives of the A4L from that focus group were transcribed and were compared to the student responses from survey and reflective documents.

\section{Action Research Findings}

Anderson (1998) suggested a general qualitative research approach for analysis of cases that "organizes the data into descriptive themes" (p. 158). This study is organized according to this strategy suggested by Anderson. The themes and descriptive analysis of data were gathered from transcribed interviews, survey results, and student documents. 
The interlocking and overarching major themes of Effort and Closing Gaps, and the underlying theme of Fair Ranking issues appear within discussion of each of the research questions, and are presented along with identified sub-themes. Sub-themes associated with Effort were: Metacognitive Effort (RQ \#1); Cultivating Persistent Effort (RQ \#2); and Effort as Responsibility (RQ \#3). Subthemes associated with Closing Gaps were: Closing the Engagement Gap (RQ \#1); Closing the Assessment Gap, Closing the Feedback Gap, and Closing the Analysis Gap (RQ \#2); and Closing the Cultural Gap (RQ \#3). The major theme Fair Ranking was an underlying issue throughout the focus group discussion, reflected in concerns of parents for their own children, but appearing most clearly in discussion of Research Question 3.

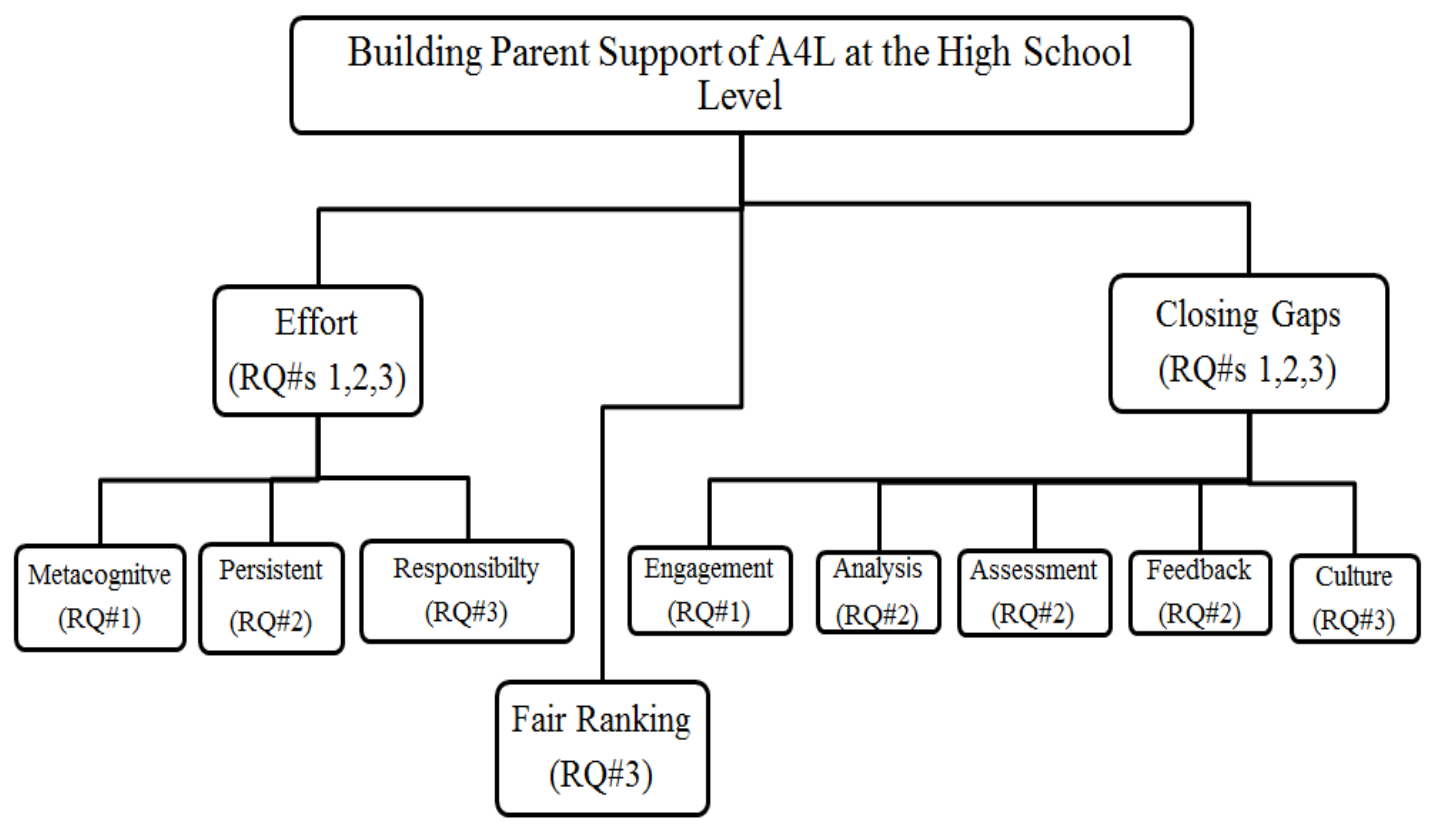

Figure 3: The Study's Themes and Subthemes 


\section{Research Question One}

Research question one addressed what attracts parents to supporting Assessment for Learning (A4L) reforms at the high school level. At the first data collection opportunity in December 2012, parents were invited to an information night to understand study guides used to prepare for finals, meet teachers of the freshmen curriculum, and begin to prepare their students for their first encounters with taking tests at high school.

In terms of CBAM SoC, those in attendance for the informal presentation started at Stage Zero with little knowledge. Only $27 \%$ of the parents surveyed identified that they knew the difference between formative and summative assessment before the presentation. After reviewing the main pillars of formative assessment, including addressing learning targets, providing feedback, and promoting activities of selfassessment, parents had already moved onto Stage 1 of CBAM SoC, Informational. Survey results tabulated from the 105 surveys indicated 59\% of parents shifted toward better knowing the difference between formative and summative assessment, and $94 \%$ of parents already were informed/convinced that while studying for finals, students must assess their own strengths and weakness in terms of the course objectives.

For some parents that night, the concepts presented were taking a personal twist, thus beginning Stage 2, or the first reflective stage, of the spiral CBAM. Only $20 \%$ of the 105 parents attending the presentation indicated interest in the focus group opportunity. After semester grades were sent home in January 2013 and by the time the focus group information was provided, only seven participants volunteered their time in February 2013. The sub-themes of Metacognitive Effort and Closing the Engagement Gap were attractive and personal features for parents supporting A4L. 
Metacognitive effort. The results from the 2010 Asset program survey were shared with the focus group to begin the conversation and to frame the A4L reform. Two patterns were identified from the survey results. The first pattern was the decline in positive student responses as students matured, to questions involving parents and their expectations regarding coursework. The second was the strong correlation between those expectations from parents and the parents' support. For both patterns, parents collectively addressed that the age of the students matters. Parents are attracted to the efforts of $\mathrm{A} 4 \mathrm{~L}$ as it recognizes the socio-emotional growth of their child and encourages intellectual maturity. As one parent mentioned, their freshmen come to them for everything since they seem to be grounded in their parents' ideology as they expect most freshmen are. Another parent agreed to that statement adding that, "Parent support at the freshmen level is homework help or help studying for tests." With an older high school student, however, it is not that cut and dried. "The work becomes more difficult and thus the student must get more engaged in the learning," added a parent.

Adding to the metacognitive effort discussion, the 2010 Asset program survey results indicated that for most questions, the freshmen year statistics are the most positive, dropping significantly by junior year, and as one focus group member mentions, "By senior year, some of the data bounces back. I wonder if they got more retrospective ...maybe that is due to college conversations." Call it retrospective or perspective; it is impressive how the data for questions relating to satisfaction with grades and preparing for success (with either homework or tests) mirror each other. Freshmen year is the highest with $68 \%, 56 \%$ by junior year, and the bounce back to $63 \%$ by senior year. 
"Maybe students think it should be easier, even though they did not place much effort into it." Agreeing another parent adds, "My son thinks he is trying."

Parents assumed that students should start to be more self-sufficient by the time of high school and that the junior high environment may be too coddling to prepare students for the reality of mustering up some effort and getting the job done. Parents in this study understood that freshmen students will not think for themselves nor will they seek out help on their own. This stumbling block, noticed by parents after the first month of school, is the harsh reality that they need to figure it out on their own. "It is a part of life," one parent added. The accountability for their own actions and the maturity to realize it is the key part of how A4L can help all students. It is an attractive feature that embraces the growth of students both mentally and physically but also invites parents to be "a catalyst in this particular stage of life" for their students, as one parent stated.

Closing the engagement gap. From the student self-assessment documents collected during the first semester of the 2013/2014 school year, biology students provided reflection and feedback regarding aspects of their academic success in the class. Of the 71 documents reviewed, 34 students included specifics to the question regarding the student's plan for improvement that would involve parents. The most frequent response from the students was "help me study" by methods of quizzing them from a review study or reviewing flashcards. Six responses specifically requested help with homework, thus confirming what the parents knew -that freshmen students do seek help, but the student responses were not specific in their requests.

The suggestions, solicited from students, for parents to become more engaged with their teen were listening to them, encouraging them to do their best, or helping them 
get to school early for extra help. However, it was the student responses denoting that parents are not involved with the phrases of "can't help," "won't help," and "really nothing can be done" that stood out during the review of self-assessment documents. These phrases added to the following parent comment to put this gap into perspective; "For me to help, I would need to relearn some things. And who wants to go back and learn that...even though it would not take much." Parents are scared to become involved with the academics in high school and need a course of action to become more engaged. A4L does not put parents in a position to learn course material but in a role to facilitate their child's reflection of their own learning. Formative assessment's reflective nature, of what students know and what they do not, is attractive to parents as they can become engaged as moral support for their children. Students recognize the parents' standoffish behavior not their parents' support and care. Once schools can help to close the engagement gap, it is one step to better understand the value parents place on assessment reform at the high school.

\section{Research Question Two}

While addressing question two, the study and its participants were engaged at the Stage 3 level of concern of CBAM, managing the task of assessment reform. Parents were aware of the reform and found a personal attraction to it, and now needed to better apply its goals. Responses related to research question two noted that for parents the best way to encourage the efforts of students with formative assessment was to better understand gaps in assessment, feedback, and analysis. The sub-theme cultivating persistent effort elaborates the major theme of Effort. Once focus is placed in these areas, parents can begin to value the goals of $\mathrm{A} 4 \mathrm{~L}$. 
Closing the assessment gap. One focus group question aimed to identify the assessments targets within District Alpha schools from the parent perspective. From a list of 19 verbs, each focus group participant chose five assessment terms that best described their child's experience thus far in high school. From the 31 choices collected that evening, the verbs of explain, solve, define, recall, and summarize were pinpointed as the most identifiable assessment terms. Weaker responses included verbs of apply, compare, contrast, and analyze. The assessment terms absent from the parents' viewpoint were predict, estimate, and formulate. An eye-opening moment of the night was when the list of verbs were overlaid with the top 12 verbs identified by the ACT Company as the assessment targets for curriculum and instruction leading to future academic success. These verbs are listed Table 3.

Table 3

The ACT's Top Twelve Assessment Verbs

\begin{tabular}{llll}
\hline Trace & Analyze & Infer & Evaluate \\
Formulate & Describe & Summarize & Compare \\
Contrast & Predict & Support & Explain \\
\hline
\end{tabular}

Of the top five targets given by parents, only explain and summarize matched, while analyze, infer, compare, and contrast were recognized by parents but not given top billing as the assessments provided at District Alpha. As one participant stated, "Explain can be applied to any course taught at the school addressing any topic and the same goes for summarize. Solve or formulate is most applicable to math, and English is your analyze, support, and refer." Another parent responds, "We know there is a focus on 
vocabulary for the district and that matches why define was one of the words most picked." The format of assessment was then addressed with one parent's comment, "This is hard when 95\% of the tests are bubbles. How can you use these words (i.e., ACT's list) on bubble test?" Adding to the test format comment, another parent in the group pointed out, "Yes, Scantron tests are easier for the teachers but it does not allow the students to demonstrate what they really know." Parents were aware of Common Core curriculum adopted by the state for English Language Arts and Mathematics and were told of science's future with the Next Generation Science Standards. Specifically focusing on science, the Next Generation Science Standards were created with the similar focus of ACT's assessment verbs of obtain, develop, and analyze. The choice of verbs unnerved most of the parents attending the focus group session. Unanimously, it was stated that this list of verbs would be way too difficult for their freshmen student. As one parent summarized, "I know they can receive information and process it. But for them to develop and to analyze, what is the level of complexity expected?"

Branching off the comment of student processing, a parent mentioned the use of technology (i.e., cell phone, computer, or tablet). Technology can help address the highorder verbs put forth and promoted by ACT. One such computerized tool is on-line simulations. These programs have students manipulate a scenario and learn the concepts of the course through experience. "Yes, the computer is the answer but they would need stronger teaching and be able to have them ready for this kind of learning." Another use of technology is applications and podcasts, especially used in a flipped classroom. Asking the group to gauge the student effort placed into this form of assessment, the group had a unanimous answer of 'no' for this approach. Even though the premise of a 
flipped classroom is for students to assess what they just learned about the concepts and then prepare/formulate responses or questions related, it seems too unrealistic in the eyes of the parents participating in the focus group. The overwhelming feeling was "this is so different from when we grew up!" with the addition of, "they [students] are conditioned to sit and get. So how does this work?" Parents noted the gap is assessment expectations which limits their comprehension of $\mathrm{A} 4 \mathrm{~L}$ and questions the learning process.

Closing the feedback gap. It is not a goal of A4L to simply sit, get, and dump the information. Instead, formative learning and assessment is a process of strengthening the metacognitive skills of students through reflections on their learning. One parent recognized the merits of $\mathrm{A} 4 \mathrm{~L}$, "Children want the book closed [no reflection after an assessment] but I would rather want them to realize 'do you know what you got wrong'...that is important to me." This prompted another parent to ask, "Do teachers go over tests? Do they take the time?" Giving the opportunity for students to review their most missed question, not necessarily the most missed, was valued by the group. One parent reiterated,

Not even going over the most missed questions is appealing to me because my kid may not miss those questions and they never get a chance to understand what they got wrong. It is more individual so their review should be also.

Individual attention, particularly as feedback, is specified in the framework of A4L and is one way to encourage more parent support.

The efforts placed in feedback are crucial to parents valuing A4L. Parents should be happy that students responded positively $(\sim 73 \%)$ that they learn a lot from feedback on their work based on the 2013 5Essentials survey. Affirming an already shared opinion, one parent stressed, “I still don't care about the questions that everyone else got wrong. I 
care about what my student did." One of the instructional strategies presented to District Alpha's teaching staff in 2010 was providing frequent feedback to students about their learning related to the objective within 48 hours. The district continued to incorporate another learning strategy in 2012 of explicitly teaching learning skills and strategies using graphic organizers for patterning, thinking and writing to help close the feedback gap.

Closing the analysis gap. "There are three things extremely hard: steel, diamond, and to know one's self" (Ben Franklin, 1750). Parents want their students to do the "heavy lifting" of their education and they seem to agree with McMillan and Hearn's (2008) emphasis that self-assessment needs to identify the discrepancies and evaluate progress. While parents like the reflective check-in points of formative assessment, the steps necessary to become proficient require more assistance with analyzing, such as identifying a cause to an effect. Already seen as a gap in high school assessment, analysis is also noted by parents to be lacking at elementary districts. The junior high environment with double period class times promotes most work to be completed during the school day. The effort to analyze or spend more time is difficult to value. As one parent reemphasized, “They [students] can't assess their quality of work!” It cannot be expected that students can automatically self-assess and be able to reflect upon their progress. One parent reflected, "Lead them through the process. Then they can do it." All participants agreed that by senior year the expectations of A4L, especially selfassessment, will be productive and possibly be used as a strategy in college.

The students' response on self-assessments collected from Biology classes correlated to the parents' opinions. Freshmen truly do not have a good grasp on analyzing. Some student responses to ways to improve in biology class included a more 
holistic approach stating, "Improve on the things I don't do," as well as a more specific approach stating, "Making sure I complete all the homework assignments and paying complete attention during class." The mentality of young teenagers appears that they are invincible and their comments of "I don't have any strengths or weaknesses," "I'm going to keep doing what I'm doing," or "I am good at everything I do, I'm already the best," support that notion. These students do not care, do not take studying seriously, or do not know how to reflect upon their learning.

Cultivating persistent effort. The previous student remarks are examples of the attitudes that parents face in order to become full supporters of A4L. How can formative assessment practices flourish if students struggle with effort? From the student selfassessment documents requesting an effort self-rank, the lowest value on a scale from 110 was a 5/6, with the reasoning that they just did not like the homework or simply, "This class is not as fun as the others." For the effort ranks between 7 and 9, students gave their ranking based upon their efficient homework completion, paying attention during class, and the 'could have done more' excuse. Only one student said their effort on the class was solely based on wanting to get a good grade. The 10 out of 10 rank mirrored statements such as "I do my work," "I participate in class," and "I try my hardest in everything." Within the 5Essentials survey, students were asked if they understood that you have to work hard to do well. At least $71 \%$ of the students who were surveyed noted this significance. For another 5Essentials question, targeting the similar concepts of hard work and application, $87 \%$ of students countered that they agree with the statement that working hard in high school matters for success in the work force. In addition, $75 \%$ of the students affirmed that what they learn in class is necessary for success in the future. 
Also included on the self-assessment documents was a question asking for reflection on how students could improve their Biology grades. The overwhelming response from one third of the students was the phrase "study more." What does that mean specifically? One student added to their comment, "Studying. That is really important!” A parent addition to studying was, "My boys do need help with their academics but they would rather sit in their room with their cell phone, text, and claim that it was study time." From the compilation of the 2013 5Essentials survey, the student population responded positively (close to 69\%) to the idea that they do set aside time to do homework and study. In a later question presented in the survey, $84 \%$ of students logged realized that homework assignments help them learn the course material. However, reflecting specifically to their biology coursework, students were lost when reviewing their study packet provided by the teacher with given objectives. "I don't know what to do with it," "I don't like studying," I should study," or "Don't need to study" were some student responses. This matched the worrisome contemplation from one parent, "I think that it [helping to self-assess at home] is hard to do at home because I don't know what they [teachers] want or the course demands." This may be a reason why only $43 \%$ of students agreed that they always study for tests, according to the 2013 5Essentials survey. Ironically in the same survey, $92 \%$ of students identified it was clear to them what they need to do to get a good grade. There is a discrepancy in their effort that needs to be addressed.

Since homework is the most outward sign of persistent effort, focus group participants agreed that is it their expectation for their child to place effort into homework, even if it is not graded. As one parent stated, "If he is going through effort to 
get the homework done and the grade is not there, then that is ok. But when the grade is low and no effort is placed, then that is a problem."

Persistent effort continued to be the theme as a scenario was presented to the parents in the focus group. For this scenario, Sheila does her homework, asks questions, and Mike does not believe in homework and does not attend school regularly. Sheila has test anxiety versus Mike who performs well on tests. Yet both students earn the same grade for the class. Immediately the conversation reverted back to the theme of closing the assessment gap, and how it is not fair that the grade of a $\mathrm{C}$ is used to label both students who are obviously not the same as students. Upon returning back to the effort made by the students in the scenario, one parent posed a question to the group:

Think about after schooling; who do you want to hire? The guy who knows everything but does not do a lick of work or the person who works really hard but still does not know anything. Really, I would want a person who knows his stuff and does a really good job... a combo of the two.

Exactly, exclaimed another parent across the table, who posed an additional question to the group. "Do you have a lot of 'Mikes' at your place?" Another participant stated, "Good question. Yes, we do. But they work in teams so.....I don't know."

How do you inspire effort? In the case of homework taken as a grade, parents were on the opposite sides of the spectrum. One opinion was, "Grading homework is forcing them [the students] to do something" versus, "If homework is meant to help them learn then why is it graded? Why it is then considered a learning aid?" Debating effort in regards to learning, the answer given by one parent was "the motivated student will exceed. Everyone else, you need to fend for yourself." Earlier it was stated that freshmen are more diligent than the older students in completing a job. Students' 
motivation is a force to be reckoned with and it boils down to persistence. As one parent put it, "Doing something every day [like homework], is reality and that makes them better." As another parent added, "It is about work ethic that is their responsibility." Summarized by another,

That is not what real world is about...you can't just wait for the big test in order to produce. There is a lot of work during the week, the whole week long. You need to do all the stupid little things that make up your job.

\section{Research Question Three}

The third research question asked what ultimately affects parent perceptions and influences their support of the district's assessment for learning (A4L) initiative. At this point, CBAM's SoC Stage 4 was key to interpreting the results. A4L needs to respond to the possibility of both positive and negative consequences as sub-themes that may undermine sustainability of parent support. The positive sub-theme Effort as Responsibility would sustain parent support as parents could contribute to morphing the effort of their student into responsibility to learn. The major theme of Fair Ranking is discussed under research question three, as well as the sub-theme of Closing the Cultural Gap.

Effort as responsibility. To start the discussion on responsibility, effort reviewed by the 2013 5Essentials survey had the rating for both Academic Engagement (I work hard to do my best in this class) and Rigorous Study Habits (I always study for tests) had 'very weak' and 'weak,' respectively. Students at District Alpha do not possess a strong sense of responsibility. Schools with a strong student responsibility report that students are active participants in their learning and that they regularly attend class prepared to learn (5Essentials, 2013). Responses to questions relating to student responsibility on the 5Essentials survey at the Central campus were so low that the school 
cannot even be ranked on the responsibility scale. With an attendance rate of $95 \%$ at the school (Lincoln-Way Central 5Essentials, 2013), evidence shows students are attending classes. However, students are unwilling to assume responsibility by always turning in homework, actively participating in class activities, or regularly paying attention in class. Supporting the existence of the lack of responsibility appeared on the self-assessments, with $100 \%$ of the responses indicating that students should improve in completing a review packet before it is due, coming in for extra help, or studying. It seemed parents know the students' weaknesses and anticipated their responses. Believing their child can be responsible enough to undergo an adjustment in assessment practices is a positive influence of parent support. However, until the student assumes more responsibility, it is difficult to move forward with the goals of $\mathrm{A} 4 \mathrm{~L}$ and be able to judge the fairness of the reform.

Fair ranking. The third cross cutting and overarching major theme emerged from the data analysis for the third research question. The repeated outcry for fairness was especially apparent when discussing that "American Education would be best served by an integrated system where summative and interim formative components are built from common frameworks " (ETS, Pearson, \& College Board, 2010, p. 7). To this parents, responded with their own suggestions for an integrated fair system. After joking about not telling their percentages of summative versus formative assessment without others telling their percentages first, the group's consensus was the formative and summative assessment paradigm must be an indirect relationship. Some parents suggested a 70/30 split, a 50/50 because "Who should get more out of formative but to be completing objective, then you need to also go with summative," or simply put, "The 
lower percentage on summative, more learning by doing is now the goal." Parents value the opportunity for students to evaluate the quality of their work and their learning and supporting efforts to be fair. Parents believed that using self-assessment is a fair practice as it relates to a student's motivation. If the student is motivated to do better, they will reflect and find a better way. Parents note that formative assessments allow for fair ranking of what students have learned. Summaries from the parents regarding formative assessments were, "My son is more likely to push back and find out why, and that is the inquiring mind," and "Formative assessment because it opens the door to show your ability. Some are not born with the straight linked mind. It depends upon the student personality." However, targeting the subjectivity of formative assessment, it challenges the more traditional view of assessment, thus indicating another reason for closing the assessment gap. Is it fair to assess all students the same as in the scenario between Sheila and Mike? Parents identified a struggle between 'the way it was' and 'the way it should be.' As revealed in their conversations, the parents began to lean to the less traditional side of academia expressed by more empathy for Sheila, who is struggling with comprehension, but works hard and only earns an average grade. Can this new perspective be supported nationwide so students at District Alpha do not lose rank behind other high school students? This concern about a diluted emphasis on academic achievement is seen as a possible consequence supporting $\mathrm{A} 4 \mathrm{~L}$, and therefore deters parent engagement.

Closing the cultural gap. This conflict between 'the way it was' and 'the way it should be' is the final gap influencing parent's support. It is one of culture. Considering the cultural gap focuses on the difference between parents and students, and the battle 
between the past and present expectations of schooling. Should a school culture focus on the learning outcomes of the Assessment for Learning (A4L) reform if the consequence would be devaluing grades? Starting with the conflicting expectations of valuing a grade or understanding the concepts, from the focus group discussion six out of the seven parents believed that their students value the grade more than the understanding. Supporting this idea were phrases like, "I think he is worried about the grade since he has to answer to mom and dad," and "When am I going to use this again, this is stupid, so he studies to get it over with." One student response from their biology class reflection agreed to this sentiment of pursuing the grade, with the student saying "Doing better on the labs [is more important] because they are worth like a lot of the grade."

From the parent perspective, the conflicting viewpoints were expressed clearly by one parent who agreed that the learning and understanding is the ultimate goal, but continued to have an internal conflict with 'the grade' since high school is not the last educational step. "I wish I could say that I would be all right just to say I'm OK with learning the material, but society does not allow that. Everything is based on grades." Influencing the parents' support of A4L is the cultural struggle between supporting A4L and guaranteeing their child is on target for the next step in their educational career and promoting new academic ventures. The parents were more comfortable with the past, a culture of grades, than with the promise of a culture of learning. As one parent acknowledged, however, "There are people that can learn the material the night before and take a test. But then they forget the day after. So they can get the grade but do they understand later on? I want them to learn." An optimistic student response from a student self-assessment document stated, “To improve I need a better understanding of 
the subject. Hopefully that will eventually come with practice."

Regardless of what A4L activity was promoted, the comfort level of parents ultimately won in determining the parents' support. One example was at the end of the informal presentation when the parents were aware of the impact of A4L for their child's learning in high school, but only $11 \%$ volunteered their names in order to be invited to the focus group interview. The other example was the focus group itself. The parents appeared to be positive and supportive, but the parents' survey responses to remaining a partner with the district during its assessment reform were stagnating and neutral. The majority of parents were on the fence about talking to their children about their study habits. Only one parent would talk about A4L with other parents, while another disagreed about sharing their excitement to other parents. All the parents were unsure of their position (choosing neutral) in talking to teachers about A4L. Frustrating, yet understandable, it demonstrates that the comfort level of parents with A4L drives the cultural change. At this point, the study hints at teacher and parent collaboration, which is stage 5 of CBAM's SoC, but reality has still not risen to that level.

\section{Research Question Four}

This question was designed to consider the research from a different angle than the first three questions that were focused on understanding parent values and perceptions. The last question raised the issue of how educational leaders can sustain home/school collaboration during the Assessment for Learning (A4L) reform process? It is not traditionally accepted for parents to collaborate with the teachers at District Alpha. In addressing research question four, parents were unsure how educational leaders can sustain home/school collaboration during this assessment reform. It is obvious that 
parents are not looking for 'cold' professionals and would prefer teachers who develop a 'personal touch' in communication and relationships as Graham-Clay (2005) would suggest. The parents involved in the focus group hinted more at communication, especially in addressing specific feedback to their student's learning. The teachers at District Alpha are perceptively aware they are standing in a neutral gear in regards to working with families to advance the school's mission to maximize the academic and social growth of all students. It was suggested that the school can regularly communicate with parents about how they can help their children learn. It is apparent that the first steps in maintaining a two-way communication street must begin with the district and its staff. Specific suggestions for practice that emerged in the study will be shared in a section of Chapter V.

\section{Chapter Summary}

Inspired by the living theory theme of "how do I improve what I am doing?" this action research explored the possibility of earning parent support for A4L. By using several methods of data collection, including surveys, a focus group, and document analysis, the data provided a synthesis of the practicalities of earning parent support. This dissertation study spanned December 2012 through December 2013 and introduced the concepts of Assessment for Learning to parents of District Alpha to uncover their opinions and perceptions regarding the initiative. The parents revealed during a small focus group conversation, and students supported through their survey answers, how to move forward toward a solution of parental support. The major cross cutting themes of Effort, Closing Gaps, and Fair Ranking emerged as the solutions. 
Student effort, as metacognitive, persistent, and growing into taking greater responsibility, is recognized by parents as an attractive feature of $\mathrm{A} 4 \mathrm{~L}$, but also as containing hindering features. The gaps in engagement, assessment, feedback, analysis, and culture need to be closed, to shrink, in order for support of parents to be sustained and genuine. Finally, the last major theme was fair ranking. The concern of parents is how this new assessment reform can be fair, at the same time understanding it cannot be equal for each student. Through the Concerns Based Adoption Model's Stages of Concern, stages 0-4 suggested how District Alpha can transform assessment practices by adopting the process that works with the concerns of the parents. The suggestions about how an educational leader can sustain home/school collaboration during assessment reform are forthcoming in the final chapter. 


\section{CHAPTER V}

\section{IMPLICATIONS, RECOMMENDATIONS, AND CONCLUSIONS}

To accomplish great things, we must not only act, but also dream; not only plan, but also believe.-Anatole France

This chapter begins with an overview of the action research conducted at District Alpha. Thomas Guskey presented to District Alpha in the spring of 2013, so the ideas of formative assessment were not new. In 1949, Ralph Tyler discussed the basic principles of assessment to include: (a) what do you want students to learn? and (b) what evidence would be accepted to verify this? The challenge has been stabilizing formative assessment through educational transitions such as standards-based curriculum and standardized testing. One solution for stability is for educational leaders to recognize that the critical factors for change are cultural values and mindsets (Deal, 1990). This dissertation study explored how parents can play a supportive role in implementing and sustaining Assessment for Learning (A4L). From the research established by Auerbach (2009), Epstein (2001), and especially Stiggins (2007), we know the collaboration with families is vital to change schools. For it to become a reality, it is necessary to embrace, work with, but more importantly grow with the families of District Alpha.

Qualitative research studies are rarely used by high school administrators; however, for this study the action research approach was ideal. Action research focuses on an educational problem that can be solved locally in a variety of ways. Action research was the best approach for the organizational problem of needing to build parent 
support for the Assessment for Learning (A4L) paradigm. My roles as both teacher and department chair at District Alpha allowed me to craft my own living educational theory that sought to analyze the possibility of sustainability. Attending to the Concerns Based Adoption Model's Stages of Concern (CBAM's SoC), data was collected through a variety of formats. Included in the data collected for interpretation were an informal presentation, surveys conducted personally, by the district, and by the state, a document analysis, and most importantly, a focus group interview and its transcript.

The themes emerging in this study suggest that a high school's implementation of formative assessment to include parent support must build on and incorporate discussions on: how to develop metacognitive and persistent effort of students and their responsibility to learn; closing gaps identified in engagement, assessment, feedback, analysis, and culture; and approaches to fair ranking in this era of continuing high-stakes testing. Implications of these themes are discussed to better understand the student's role in A4L and to designate the school's role in supporting them along the way. The promise of formative assessment rests with the school leadership team (Black, Harrison, Lee, Marshall, \& Wiliam, 2004); however, the fulfillment of that promise takes collaboration and support of everyone involved, including parents/families.

The purpose of this action research study was to identify how a high school district can initiate a partnership between home and school in order to support high school students through Assessment for Learning (A4L) changes. The decision to facilitate an action research project was inspired by my wanting to know the story behind the results of a 2010 Asset program survey. Students were not happy with their grades, but they were confident they would pass their classes. Students were dissatisfied with 
their grades, but the percentage of homework completion declined after freshmen year. My curiosity increased after completing my 2011 pilot study which garnered positive feedback and found that parents favor educational reforms at the high school. However, the challenge was implementing sustained support.

I imagined a solution to the implementation problem, and I explored my solution to continue to seek parent support using a living approach to educational theory affirmed by Whitehead (2008). This action research study resulted in answers to the following questions:

1. What attracts parents to supporting Assessment for Learning (A4L) reforms at the high school level?

2. How can parents value assessment reform and comprehend the goals of Assessment for Learning (A4L)?

3. What affects parent perceptions and influences their support of the district's Assessment for Learning (A4L) initiative?

4. How can educational leaders sustain home/ school collaboration during the Assessment for Learning (A4L) reform process?

\section{Implications}

Educational research is undergoing major advance that will further deepen our theoretical understanding of process in cognition, learning and teaching... strengthen our ability to contribute to educational practice. (Greeno, Collins, \& Resnick, 1996, p. 15)

The three thematic issues of cognition and learning addressed by Greeno, Collins, and Resnick (1996) are avenues for discussing implications of the findings from the triangulation of the research data for this study. Those thematic issues are the nature of 
knowing, of learning and transfer, and of motivation and engagement. These themes align with the CBAM's descriptors for each stage of concern reviewed in Chapter II. Research Question One sought to understand the initial attraction of the A4L movement to parents and reflected what the parents already knew about A4L. Its findings best addressed the awareness, informational, and personal concerns of stages zero, one, and two, respectively. For Research Question Two, the nature of learning and transfer is applicable to stage three, management. Within management, parents learn to appreciate the goals of A4L and begin to transfer their knowledge and validate their understanding of formative assessment. It is from Research Question Three that we see the sustainability of the home/school support depends upon the engagement of the parents and how motivated they are to deal with the consequences that stage four suggests. Research Question Four yielded insights into stage five's collaboration emphasis as well as suggestions for educational leaders that are discussed under Recommendations.

\section{The Nature of Knowing}

Greeno, Collins, and Resnick's (1996) summary of the nature of knowing appropriately aligns with the first few stages of concern (SoC) established by the Concerns Based Adoption Model (CBAM) that are captured in the first research question. Playing into what both parents and students know about formative assessment, or their schemas, helps identify what attracts parents to support A4L at District Alpha. The themes of metacognitive effort and closing the engagement gap emerged from data collected regarding the first research question. The students' effort placed in learning and the opportunity for the parents' engagement in their learning are the attractive features of A4L reform. 
A component of $\mathrm{A} 4 \mathrm{~L}$ is that students must be able to critically evaluate their own knowledge and skill levels. The metacognitive ability of the students was a concern for the parents. Parents worry whether freshmen students have the capacity to reflect upon their thinking and, if not, what supportive roles are available to them during the reform. The best answer is the relationship and time spent with adults. Weaver (2007) concludes that this valued time is important in developing good decision-making skills. Families continue to possess the most potential to spark growth from teenagers, a conclusion emphasized by Fehrmann, Keith, and Reimers' (1987) direct correlation of higher grades with parental support. I believe to close the engagement gap could be as simple as addressing this reality: parents do not know their role as change agents and students are unaware of their family's potential. It is finding balance between independence and hand holding as the student transitions into high school. It is impossible to assume that a family's care for their child dissipates during the summer between eighth and ninth grade. Freshmen students need homework assistance when possible and encouragement given by families always. Self-assessment is a doable task for freshmen; it is just a matter of having support to make the task more manageable. A4L makes it possible for students and their families to become more collaborative and bridge the expectations between middle and high school.

\section{The Nature of Learning and Transfer}

Greeno, Collins, and Resnick (1996) outlined the cognitive perspective summarized as learning and the transfer of knowledge. Data interpretation regarding Research Question Two found that parents need: (a) to learn more about improving analysis skills, assessment goals, and feedback; and (b) to better understand their teens' 
lack of results-oriented persistence. Once the gaps in those areas are better addressed, parents will be able to transfer their support onto other areas.

Research Question Two's findings complement each other and are best summarized as, "All students must believe that they can succeed at learning if they try" (Stiggins, 2005b, p. 326). One step toward success is for teachers to incorporate learning objectives. McMillan and Hearn (2008) remind educators that goals and sub goals will help evaluate academic progress and, in doing so, will increase students' persistence toward a greater goal, hence providing motivation. The goals used to evaluate progress within District Alpha were the first instructional strategy implemented in 2008. 'I Can' statements address the day's lesson and are mentioned at the beginning, middle, and end of each class every day. The curriculum, instruction, and assessment of District Alpha will continue to incorporate this strategy. Identifying these statements helps create assessments, direct feedback, and encourage analysis to motivate students to be more persistent with their learning. Persistent effort guided by learning targets will be necessary for future assessments, especially in science.

The focus group parents identified District Alpha's assessments at a lower level of knowledge and comprehension than the higher level presented within the Next Generation Science Standards. With parents becoming more aware of these targets presented in each class, they can evaluate the assessment focus as either high or low level. Parents can discuss more opportunities for implementation of higher order targets, such as those used most often on standardized tests. Parents should encourage more assessments using the skills of construct, predict, and evaluate, even though these assessment goals seem somewhat impossible with today's available technology. 
One parent subtly mentioned technology's (cell phones, computers, apps) role in the assessment gap. The possible absence of self-seeking answers also furthers the gap in persistent effort seen in high school students since technology provides instant gratification, especially with checking the Internet. Nonetheless, parents should be pleased with how students responded on the 5Essentials survey-agreeing that their classes make them think $(81.1 \%)$ and teachers at District Alpha want students to become better thinkers, not just memorize things $(87.7 \%)$. Still, the "heavy lifting," as one parent mentioned, must be done by the student. As reassurance, Rolheiser and Ross (2001) remind us that "students who are taught self-evaluation skills are more likely to persist on difficult tasks, be more confident about their ability, and take greater responsibility for their work" (p. 9). Since 2009, District Alpha has engaged students in more reflective activities on their learning. To be more transparent about the learning process, these documents help students realize the attainable goals for lessons and reassurance checks on persistence. These reflective checks can help transition parents into better understanding the learning process and help gauge the efforts of their child.

Self-assessment can only be beneficial with feedback. "Learners should be provided with periodic feedback on their performance in the course" (Sitzmann, Ely, Brown, \& Bauer, 2010, p.181). A more individualized plan for education was appealing to parents. One way to continue to address this concern is better implementation of the instructional strategy of feedback. One of the later implemented strategies for District Alpha in 2010, feedback to students must be prompt, concise, and specific to the task at hand. Stiggins (1999) noted feedback can help students see, understand, and appreciate their academic journey. Guskey (2008) identified feedback to include the different 
presentations of concepts and different forms of student engagement to provide a more successful learning experience. Receiving the right feedback will address the analysis gap discussed in Chapter IV.

\section{The Nature of Motivation and Engagement}

The third cognitive theme presented by Greeno, Collins, and Resnick (1996) is motivation and engagement with the information to be learned. Required for this cognitive transition is active participation, and its importance was most evident during the focus group. The opportunity to have someone listen to their opinions seemed energizing. The parents' enthusiasm to support A4L is influenced by fair ranking, responsibility, and most importantly trust. Parents desire a more responsible student. Responsibility should not mean always earning a good grade. Students can become more responsible to their learning needs by working within the realm of their personality. As parents spoke of honoring individuality, personality tests like Myers-Briggs came to mind. These can be used to encourage students to be in touch with their individualistic learning style. Pinpointing the characteristics of their child, parents can also benefit from recognizing those characteristics to help address ways to improve the efforts correlated to learning, motivation, and receiving feedback. Highlighting these points can make A4L more attractive and engaging for parents.

Ultimately, I view the parents' motivation, engagement, and buy-in of A4L as dependent upon trust. Schools must develop social trust as stressed by Gold, Simon, and Brown (2005) and cannot ignore an environment of mistrust. The benchmark score for the school was 'very strong' on the measure of Teacher-Parent trust, as reported by teachers (Lincoln-Way Central 5Essential, 2013). The teachers report that they feel good 
about parents' support for their work, and they work hard to build trusting relationships with parents. The teachers note the respect from the community, and teachers respect families as they do their best to help their children learn. As for the students' point of view, over a majority of students agree that the teachers at the school respect their culture and the experiences of all their students. Unfortunately, from personal experience as department chair for science, I would say parents do not always have reciprocal trust for the school. When a parent initiates a conversation with me, and not the classroom teacher, regarding a classroom situation, it always boils down to the fear of retaliation, that the teacher will take the parent's concern out on their student.

What promotes the disconnect between the trust felt by parents and that of the students? Hoover-Dempsey et al. (2005) found that parents feel alienated from school districts when changes occur without proper parent notification. During the 2013/2014 school year, District Alpha's Board of Education emphasized improving parental and community communication opportunities. This goal included the expansion of costefficient ways to keep parents and community members up-to-date on district information and events mostly through electronic communications that include an e-newsletter, Facebook, and the website. Any related information in appropriate formats can be helpful and, in 2014, it is duly noted that electronic information is alive, well, instantaneous, and just possibly building small amounts of trust between the parents and the district.

The engagement gap previously identified is also applicable to trust. Parents will invest more time and effort and make assessment reform a priority when they are vested. As seen by the 2013 5Essentials report, the percentage of parents participating in the 
survey was close to $10 \%$. There were many reminders to voluntarily participate in the survey, such as the website, an e-newsletter link, and an email blast to the parents' email accounts. Parents rank providing their feedback and opinion of the school low even though parents want a fair shake for their students.

\section{Recommendations}

Recommendations for practice resulted largely from findings in response to Research Question Four: How can educational leaders sustain home/school collaboration during the Assessment for Learning (A4L) reform process? Activities that teachers and administrators can implement will be highlighted first, followed by recommendations for future research. Parents did not directly say what administrators should do in order to sustain home/school collaboration. The sample size of the focus group was small, yet it was a fruitful beginning. Their feedback fueled the suggestions for both teachers and administrators. These suggestions call for a better mastery of teaching skills, a promotion of different types of assessments, and more opportunities to collaborate.

\section{Recommendations for Practice}

Teachers. The parents mentioned, without knowing it, some of the district's instructional strategies - particularly learning objectives/targets, feedback, and selfassessment opportunities. The suggestion for teachers is to continue to apply these instructional strategies, along with the others, and become proficient at them. I do not think that District Alpha falls in rank with the districts evaluated by Boyle and Charles (2010), Carless (2005), or Cheng, Andrews, and Yu (2010). It is just a matter of time before these strategies become more engrained in District Alpha's culture and utilized at a mastery level. 
Infiltrating the culture is the new evaluation tool commissioned by the Illinois State Board of Education's passing of Senate Bill 315. Beginning the 2014/2015 school year, teachers will need to become more accountable with their efforts of effective teaching. Effective teaching needs to strongly emphasize a coaching aspect in order to raise students' effort to the higher expectations in the classroom. Teachers are encouraged to compare the learning targets used in their instruction and to balance the presentation of both forms of assessments (summative and formative) in their classes. Parents were nervous about Scantron tests and their purpose with assessment. Teachers need to reflect upon which type of assessment is best for the required task to ease the concerns of parents. One assessment approach is to common assessments per departments by 2016/2017, something Schmoker (2004) targeted as the vehicle to improve teaching and learning.

The emphasis on creating common assessments will also help unify feedback, another concern from parents. In the evaluation tool's first domain, the teacher will be evaluated by their recognition of the students' diverse characteristics, and their plan to provide appropriate feedback regarding the students' learning related to the objectives/standards presented in the course. The stress on feedback is also a good opportunity to encourage more analysis and self-reflection. Parents want teachers to coach students through the high-order thinking skills in order for them to be utilized correctly. The teacher will also need to target the learning objectives and appropriately communicate them to both parents and students to receive high marks on their evaluation. Overall, the acknowledgment and encouragement of growth as an individual of each student is stressed and requested by parent concerns. 
It is exciting to note the new evaluation format encourages and promotes $\mathrm{A} 4 \mathrm{~L}$ as "not just for students." The teaching staff must be open to frequent check-ins to evaluate and direct their instruction as Popham (2008) proposed in his reflection on formative assessment. Teachers must be willing to peer coach, peer evaluate, and peer reflect. One way to reflect and respond to the concerns of the parents is the A4L teacher teams established at each of District Alpha's schools. Meeting throughout the school year, this forum provides teachers the opportunity to address the suggestions made by parents but also build a network of resources. I would suggest that teachers at District Alpha do not take this new evaluation lightly. This diagnostic tool is intended to improve the level of teaching. It is quite possible that once teaching improves in the direction most hoped for by the parents, trust will follow. Trust will increase with more communication, especially if it is specific and transparent. Parents need to know that their child is not just a seat in a class.

Administrators. The first suggestion for the administrative team aligns to the new evaluation process. Administrators should dive into more of an instructional leader role, not just be a manager. To best evaluate the teaching staff, time and effort will need to be placed in knowing their department's curriculum, their creation of differentiated assessment opportunities, and their efforts placed into specific feedback. Formative assessment is not a singular event; it is a process. Being proficient in this instructional role will take time.

Administrators should seek those opportunities, either face to face, virtual, or a blended approach (Partnership for $21^{\text {st }}$ Century Skills, 2009), since communication is referred to as means to build trust. Whatever method is used, "We must evolve from the 
practice of encouraging only information distribution, because we know from psychological and motivation research on adult learning that just handing out information to adults doesn't work in terms of learning and growth" (Mapp, 2011, \113). The best suggestion is more interactive opportunities. During the focus group, parents found it refreshing to have someone listen to their responses. Possibly integrating more opportunities for parents to sit and listen to each other could happen with the return of the Parent University. Previously held in the past, the district would present topics to inform and discuss with the families in the community. Maybe this is also the solution to raise the parent's response rate on future 5Essentials surveys. Creating opportunity for interaction supports understanding the story behind the survey results and trust is promoted.

People have feelings, concerns, and questions. People learn best in situations where they can practice the skill, where they can talk about the skill with others, and where they can build a network of people in which they share and exchange information. (Mapp, 2011, I13)

An administrator's suggestion to minimize the assessment gap is to help eliminate the parent's conflict between wanting their student to understand course material instead of simply earning a good grade. Research based opinions on assessment highlight that some parents prefer assessments that make them think and encourage competition (Mu \& Childs, 2005), while others hate how assessment raises anxiety and nervousness of students (Barksdale-Ladd \& Thomas, 2000). With the conflict removed and pressure from a society perspective, the parents can be more supportive of the A4L initiative. Steps to consider include removing the negative stigma of competition. Administrators must promote intrinsic motivation by looking more at individual growth instead of the 
ranks of student achievement. The student's progression through their learning could be at least displayed as a different form for progress reports. The document attends to individualization requests made by parents. The more parents know, the more engaged they will be, thus closing the engagement gap addressed. To take it one step further in breaking a tradition, the board of education could consider the elimination of the valedictorian/salutatorian for a graduating class. A neighboring high school district of District Alpha eliminated class rank, valedictorian, and salutatorian. Instead they have adopted a system of Cum Laude recognition based on the following GPA requirements: Summa Cum Laude 4.0, Magna Cum Laude 3.99-3.75, and Cum Laude 3.74-3.5. The pressures to compete, exceed, and surpass others is gone. This is the ultimate in fair ranking.

It is time to consider a family engagement policy for the district. Creating a network, especially a social one, encourages an interaction with the school and its community. States such as Ohio, California, and Pennsylvania have required family engagement policies, and Illinois has been considering the same. With the October 2013 published 5Essentials results, ISBE has drafted its initial ideas for a family engagement proposal. The language that District Alpha could incorporate should focus on: (a) respect, value families as partners and decision-makers in school continuous improvement planning; (b) create parent and family engagement activities that build relationships among parents, families and schools through bridging economic and cultural barriers; (c) expand more families to be involved in advisory board meetings than the selected few to converse with administrators, teachers and staff; and finally, (d) planning and implementing school-based activities such as family literacy and family math nights or 
other workshops to help parents/caregivers understand how to support academics. Any effort considered will build the schools' and parents' capacity to improve student academic achievement.

\section{Recommendations for Further Research}

Being able to completely utilize the entire CBAM change model is one recommendation that I have for future research. This study yielded limited data and a way to correct it would be to include a larger sampling size. Participants in this study were centered in the high school. Efforts in future research should be made to include parents at the middle school and possibly the elementary level. Common Core and Next Generation Science Standards start as early as kindergarten, and it fruitful to consider the implications of assessment at those levels as well. The standards provide an opportunity for another study to be more longitudinal. The benefit would be to keep those participants more engaged, thus strengthening the potential for more support. Overall, building support from parents is like retaining a customer; focus on their interest and aim to please. Any future research needs to initiate a more social setting, which is appealing to parents and gets them interested in building a network.

\section{Conclusions}

We cannot build the future for our youth, but we can build our youth for the future. President Franklin D. Roosevelt while speaking about creating a better society in 1940.

The purpose of this dissertation study was to determine the degree to which parents support the efforts of formative assessment, also known as the A4L initiative. The results of this dissertation study indicate parents want to support their high school children, but there are gaps noticed by parents in the areas of assessment, feedback, 
analysis, and culture. The effort displayed by students is weak. Using the model of parent involvement adapted from Hoover-Dempsey and Sandler (1995) and HooverDempsey et al. (2005), this study's outcomes are stuck between the third level and fourth level. Parent engagement and support is stuck between the parents' encouragement and modeling of the new assessment reform and the students being able to believe in themselves as a learner and their personal motivation for learning.

The study promised to introduce the concepts of A4L. This was accomplished at the informal presentation and through the focus group. Two sources of data that best reported upon the student's study habits both at home and school were the 5Essentials survey and the self-assessment documents from science class. The focus group was the narrative piece that uncovered the opinions and perceptions of parents participating and it was most valued. Their conversations directed me to better understand where the solution lies in obtaining support for formative assessment. Per the concerns of parents, teachers can adjust their strategies to encourage a desire to learn. Finally, administrators know the value placed in creating relationships with the community.

One disappointment with this study is that its conclusions come from such a small sampling. What validates the small number of participants in the focus group is that it matches the participation on the 5Essentials survey at $10 \%$. Parents do not have an innovative role in the community; rather their role has been the 'strong but silent' partner, which was confirmed throughout this study. Parents care, but they do not care enough to step out of the shadows to be heard as individuals. The study's results mirrored the initial thoughts of parents obtained during the 2011 pilot study, and that makes sense. The parent engagement situation in 2011 was the same as in 2013; the district still does not 
have a policy on engagement, just a Board of Education goal to disseminate information. The absence of parent engagement is one limitation which I hope can be resolved in the future.

Another limitation was my naïve expectation that this time around, parents would love to participate in a study since I appealed to a more personal side with A4L's connection to final exams. Even at the end of the focus group, only one group member was willing to take the risk to be the change agent for A4L. Knowing the responses from the focus group, I was still naïve and hoped for a good response rate on the 5Essentials survey that was simultaneously open online at the time of my focus group. The lack of participation squashed the attempt to move analysis to Stage 6 of CBAM's SoC. This study just dipped its 'toe' into the reflective stage 5, seeking collaborative opportunities to have an impact in the future. Stage 6 can only be reached when the energy of parent support can increase and be strong enough to sustain the innovation. The true impact of parents is unknown and this study is just the start. The study unveiled the attractive components of $\mathrm{A} 4 \mathrm{~L}$, exposed the value of $\mathrm{A} 4 \mathrm{~L}$ to parents, and allowed parents to articulate what influences their support of A4L. It is the celebrating the small victories that will eventually move District Alpha in the right direction to seek home/school collaboration.

Schools must reconsider what, how, and why education is what it is today. Educational leaders are looking for the answer to reshape school cultures for sustained student success (Blankstein, 2010, p. 6). The standards-based education was hijacked by a testing movement and now schools need to find balance between them. Education has advanced a more unified approach at standards, and now it is time to unify assessment 
practices. The answer lies with building community. Relationships are the real work of school improvement (Blankstein, 2010, p. 67). Schools should be ready to engage parents in their districts after addressing the effort, gaps, and reflection of A4L to the community. This Assessment for Learning initiative is appealing. I know it will ultimately have benefits to student learning, and I am determined that it needs support from families to thrive.

It is my hope that this study will provide District Alpha with success in its future endeavors of building support of assessment reform. This study intended to highlight parents as the key to reform sustainability. I hope that this grassroots approach will be considered as the state continues to hold the district more accountable in developing the areas that define the district as one of the best in the state. An administrator should become more of an action researcher and seek out the opportunities to interact with the community. Seize the moment to listen to parents and truly engagement them in the process of developing a student who is prepared for lifelong learning. By understanding and leveraging the untapped potential in our learning organizations, educational leaders can bring meaning back to our schools. 


\section{REFERENCES}

Advance Illinois. (2010). The state we're in: 2010: A report card on education in Illinois. Retrieved June 18, 2012, from http://www.advanceillinois.org/filebin/src/AI-SRC.pdf.

Ames, C., Khoju, M., \& Watkins, T. (1993). Parent involvement: The relationship between school-to-home communication and parents' perceptions and beliefs (Report No. 15). Urbana, IL: ERIC Document Service No. ED362271, Center on Families, Communities, Schools, and Children's Learning, Illinois University.

Anderson, G. (1998). Fundamentals of educational research. London: Falmer Press.

Anderson G., Herr. K., \& Nihlen, A. S. (2007). Studying your own school: An educator's guide to practitioner action research $\left(2^{\text {nd }} \mathrm{ed}\right.$.). Thousand Oaks, CA: Corwin Press.

Andrade, H. (2011). Promoting learning and achievement through self-assessment. Better Evidence-Based Education, 12-13.

Andrade, H., \& Du, Y. (2007). Student responses to criteria-referenced self assessment. Assessment \& Evaluation in Higher Education, 32 (2), 159-181.

Astone, N. M., \& McLanahan, S. S. (1991). Family structure, parent practices and high school completion. American Sociological Review, 56, 309-320.

Auerbach, S. (2007). From moral supporters to struggling advocates: Reconceptualizing parent roles in education through the experience of working-class families of color. Urban Education, 42(3), 250-283.

Auerbach, S. (2009). Walking the walk: Portraits in leadership for family engagement in urban schools. The School Community Journal, 19(1), 9-31.

Baker, P., Bakken, J., Blum, C., Cates, G., Swerdlik, M., Sylvester, B., \& Thompson, J. (2007). Developing learning centered schools with a comprehensive system of student support. Illinois State University.

Baker, P., Curtis, D., \& Benenson, W. (1991). Structures and processes of planned change. The School Community Journal, 1(2), 11-19. 
Barksdale-Ladd, M. A., \& Thomas, K. F. (2000). What's at stake in high stakes testing? Teachers and parents speak out. Journal of Teacher Education, 51(5), 382-397.

Bass, B. M. (1990). From transactional to transformational leadership: Learning to share the vision. Organizational Dynamics, 18(3), 19-31.

Black, P., Harrison, C., Lee, C., Marshall, B., \& Wiliam, D. (2004). Working inside the black box: Assessment for learning in the classroom. Phi Delta Kappan, 86(1), 921.

Black, P., \& Wiliam, D. (1998). Inside the black box: Raising standards through classroom assessment. Phi Delta Kappan, 80(2), 139-144, 146-148.

Blankstein, A. M. (2010). Failure is not an option: Six principles that guide student achievement in high-performing schools. Thousand Oaks, CA: Corwin Press.

Bolman, L. G., \& Deal, T. E. (2008). Reframing organizations: Artistry, choice, and leadership ( $4^{\text {th }}$ ed.). San Francisco, CA: Jossey-Bass.

Boyle, W. F., \& Charles, M. (2010). Leading learning through assessment for learning? School Leadership and Management, 30 (3), 285-300.

Bridges, W. (2003). Managing transitions: Making the most of change $\left(2^{\text {nd }}\right.$ ed.) Cambridge, MA: DaCapo Press.

Bryant, M. T. (2004). The portable dissertation advisor. Thousand Oaks, CA: Corwin.

Bryk, A. S. (2010). Organizing schools for improvement. Phi Delta Kappan, 91(7), 2330.

Butin, D. W. (2010). The education dissertation. Thousand Oaks, CA: Corwin Press.

Caplan, J. G. (2000). Building strong family school partnerships to support high student achievement. Arlington, VA: Educational Researcher Services.

Carless, D. (2005). Prospects for the implementation of assessment for learning. Assessment in Education Principles Policy and Practice, 12(1), 39-54.

Carnoy, M., \& Loeb, S. (2002). Does external accountability affect student outcomes? A cross state analysis. Educational Evaluation and Policy Analysis, 24(4), 305-331.

Carreon, G. P., Drake, C., \& Barton, A. C. (2005). The importance of presence: Immigrant parents' school engagement experiences. American Educational Research Journal, 42(3), 465-498. 
Catsambis, S. (1998). Expanding knowledge of parental involvement in secondary education: Effects of high school academic success. Center for Research on the Education of Students Placed at Risk, Report 27. Center for Education Reform (2012). Homepage. Retrieved on June 18, 2012, from http://www.edreform.com/

Chadwick, K. G. (2004). Improving schools through community engagement: A practical guide for educators. Thousand Oaks, CA: Corwin Press.

Chappuis, J. (2005). Helping students understand assessment. Educational Leadership, November, 29-43.

Chappuis, S. J. (2007). Sound assessment through proper policy. School Administrator, 64(1), 24-26.

Chappuis, S. J., \& Chappuis, J. (2008). The best value in formative assessment. Educational Leadership, 65(4), 14-19.

Cheng, L., Andrews, S., \& Yu, Y. (2010). Impact and consequences of school-based assessment (SBA): Students' and parents' views of SBA in Hong Kong. Language Testing, 28(2), 221-249.

Child Trend Data Bank (2012). Parent involvement in education. Retrieved on June 20, 2012, from http://www.childtrendsdatabank.org/?q=node/186

Clark, R. E., \& Estes, F. (2008). Turning research into results: A guide to selecting the right performance solutions. Atlanta, GA: Information Age Publishing Inc.

Cochran-Smith, M., \& Lytle, S. L. (1993). Inside outside. Teacher research and knowledge. New York, NY: Teachers College Press.

Cohen, A. R., \& Bradford, D. L. (2005). The influence model: Using reciprocity and exchange to get what you need. Journal of Organizational Excellence, (Winter), 57-80.

Copland, M. A. (2003). Leadership of inquiry: building and sustaining capacity for school improvement. Educational Evaluation and Policy Analysis, 25(4), 375395.

Creswell, J. W. (2009) Research design: qualitative, quantitative, and mixed methods approaches. Thousand Oaks, CA: SAGE.

Deal, T. E. (1985). The symbolism of effective schools. The Elementary School Journal, 85(5), 601-620.

Deal, T. E. (1990). Reframing reform. Educational Leadership, 47 (8) 6-7,9, 11-12. 
Department of Education. (2010a). A blueprint for reform: the reauthorizing the elementary and secondary education act. Retrieved from http://www2.ed.gov/policy/elsec/leg/blueprint/publicationtoc.html

Department of Education. (2010b). Supporting families and communities. Retrieved from http://www2.ed.gov/policy/elsec/leg/blueprint/faq/supporting-family.pdf

Dewey, J. (1897). My pedagogic creed. School Journal, 54(January), 77-80.

Dewey, J. (1916). Education and democracy. New York, NY: Macmillan.

Dilley, P. (2000). Conducting successful interviews: Tips for intrepid research. Theory into Practice, 39 (3), 1-8.

Drath, W. H. (2001). The deep blue sea: Rethinking the source of leadership. San Francisco, CA: Jossey-Bass.

DuFour, R., Eaker, R., \& DuFour, R. (2005). Recurring themes of PLCs and the assumptions they challenge. In R. DuFour, R. Eaker, \& R. DuFour (Eds.), On common ground: The power of professional learning communities (pp. 7-29). Bloomington, IN: Solution Tree.

Eccles, J. S. \& Harold, R. D. (1996). Family involvement in children's and adolescent's schooling. In A. Booth \& J. F. Dunn (Eds.), Family school links: How do they affect educational outcomes? (pp. 3-34). Mahwah, NJ: Erlbaum.

Edwards, P. A., Turner, J. D., \& Mokhtari, K. (2008). Balancing the assessment of learning and for learning in support of student literacy achievement. The Reading Teacher, 61(8), 682-684.

Elmore, R. (2002). Bridging the gap between standards and achievement: The imperative for professional development in education. Washington, DC: The Albert Shanker Institute.

Elmore, R. F. (2007). School reform from the inside out: Policy, practice, and performance. Cambridge, MA: Harvard Education Press.

Epstein, J. (1992). School and family partnerships. In M. Alkin (Ed.), Encyclopedia of educational research (6 ${ }^{\text {th }}$ ed., pp. 1139-1151). New York, NY: MacMillan.

Epstein (1994). Theory to practice: School and family partnerships lead to school improvement and student success. In C. Fagnano \& B. Werber (Eds.), School, family, and community interactions: A view from the firing lines (pp. 39-52). Boulder, CO: Westview Press.

Epstein, J. (1995). School, family, community partnerships: Caring for the children we share. Phi Delta Kappan, 76(9), 701-712. 
Epstein, J. (2005). Attainable goals? The spirit and letter of the no child left behind act on parental involvement. Sociology of Education, 78(2), 179-182.

Epstein, J. (2011). School, family, and community: Preparing educators and improving schools $\left(2^{\text {nd }}\right.$ ed.). Boulder, CO: Westview Press.

Epstein, J. L., \& Sanders, M. G. (2002). Family, school, and community partnerships. In M. H. Bornstein (Ed.), Handbook of parenting: Vol. 5. Practical issues in parenting ( $2^{\text {nd }}$ ed., pp. 407-437). Mahwah, NJ: Norton.

ETS, Pearson, \& College Board. (2010). Thoughts on an Assessment of Common Core Standards. Educational Testing Service. Retrieved from: http://professionals.collegeboard.com/profdownload/thoughts-on-an-assessmentof-common-core-standards.pdf

Fan, X., \& Chen, M. (2001). Parental involvement and students' academic achievement: A meta-analysis. Educational Psychology Review, 13(1), 1-22.

Fehrmann, P. G., Keith, T. Z, \& Reimers, T. M. (1987). Home influence on school learning: Direct and indirect effects of parental involvement on high school grades. The Journal of Educational Research Journal, 80(6), 330-337.

Ferlazzo, L. (2009). Parent engagement or involvement? Retrieved on June 20, 2012, from http://www.parentinvolvementmatters.org/articles/parent-engagementinvolvement.html

Ferlazzo, L. (2011). Involvement or engagement. Educational Leadership, 68(8), 10-14.

Ferlazzo, L., \& Hammond, L. A. (2009). Building parent engagement in schools. Pennsylvania State University: Linworth Books/Libraries Unlimited.

Friend, M., \& Cook, L. (1996). Interactions: Collaborative skills for school professionals $\left(2^{\text {nd }}\right.$ ed.). White Plains, NY: Longman Publishers.

Fullan, M. (2000). The three stories of education reform. Phi Delta Kappan, 81(8), 581584.

Fullan, M. (2001). Leading in a culture of change. San Francisco, CA: Jossey-Bass.

Fullan, M., \& Hargreaves, A. (1991). What's worth fighting for in your school? Milton Keynes: Open University Press.

Gardner, D., Baker, P., Vogt, P., \& Hodel, R. (2005). Four approaches to professional development. Illinois State University Policy Center.

Glanz, J. (2003). Action research: An educational leader's guide to school improvement. Norwood, MA: Christopher-Gordan Publisher, Inc. 
Ginsburg, G. S., \& Bronstein, P. (1993). Family factors related to children's intrinsic/extrinsic motivational orientation and academic performance. Child Development 64, 1461-1474.

Goals 2000: Educate America Act. (1994). Retrieved on June 19, 2012, from http://www2.ed.gov/legislation/GOALS2000/TheAct/index.html

Gold, E., Simon, E., \& Brown, C. (2005). A new conception of parent engagement: Community organizing for school reform. In R. W. English (Ed.), Sage Handbook of Educational Leadership (pp. 237-268). Thousand Oaks, CA: Sage Publications.

Goldberg, M. F. (2006). The right leader at the right time. School Administrator, 63(10), 32-37.

Gonzalez, A., Doan Holbein, M., \& Quilter, S. (2002). High school students' goal orientations and their relationship to perceived parenting styles. Contemporary Educational Psychology, 27, 450-470.

Gonzalez-DeHass, A. R., Willems, P. P., \& Doan Holbein, M. F. (2005). Examining the relationship between parental involvement and student motivation. Educational Psychology Review, 17(2), 99-123.

Graham-Clay, S. (2005). Communicating with parents: Strategies for teachers. The School Community Journal, 16(1), 117-129

Greenleaf, R. K. (2002). Servant leadership: A Journey into the nature of legitimate power and greatness. Mahwah, NJ: Paulist Press.

Greeno, J. G., Collins, A. M., \& Resnick, L. B. (1996). Cognition and learning. In D. C. Berliner \& R. C. Calfee (Eds.), Handbook of Educational Psychology (pp. 15-46). New York, NY: Macmillan.

Gronn, P. (2000). Distributed properties: A new architecture for leadership. Educational Management \& Administration, 28(3), 317-338.

Guskey, T. R. (1996). Communicating student learning: The ASCD yearbook 1996. Alexandria, VA: ASCD.

Guskey, T. R. (2003). How classroom assessments improve learning. Educational Leadership, 60(5), 6-11.

Guskey, T. R. (2006). Making high school grades meaningful. Phi Delta Kappan, 87(9), 670-675. 
Guskey, T. R. (2007). Using assessments to improve teaching and learning. In D. Reeves (Ed.), Ahead of the curve: The power of assessment to transform teaching and learning (pp. 14-29): Bloomington, IN, Solution Tree Press.

Guskey, T. R. (2008). The rest of the story. Educational Leadership, 65(4), 28-35.

Hall, G. E., \& Hord, S. M. (2010). Implementing change: Patterns, principles, and potholes $\left(3^{\text {rd }}\right.$ ed.). Upper Saddle River, NJ: Prentice Hall.

Hanson, M. (2001). Institutional theory and educational change. Educational Administration Quarterly, 37(5), 637-661.

Hargreaves, E. (2005). Assessment for learning? Thinking outside the (black) box. Cambridge Journal of Education, 35(2), 213-224.

Hatch, M. J., \& Cunliffe, A. C. (2006). Organization theory: Modern, symbolic, and postmodern perspectives ( $2^{\text {nd }}$ ed.). Oxford, London: Oxford Press.

Heard, H. E. (2004). Family structure and adolescent school engagement: The mediating influence of family and parental resources. Houston, TX: Rice University.

Heifetz, R. A. (1994). Leadership without easy answers. Cambridge, MA: Belknap Press.

Heifetz, R., Grashow, A., \& Linsky, M. (2009). The practice of adaptive leadership: Tools and tactics for changing your organization and the world. Boston, MA: Harvard Business Press.

Henderson, A. (2004). Engaging parents, families and the community to improve student achievement. Abbott Implementation Resource Guide. Education Law Center.

Henderson, A. T., \& Mapp, K. L. (2002). A new wave of evidence: The impact of school, family, and community connections on student achievement. Austin, TX: Southwest Educational Development Laboratory.

Herr, K., \& Anderson G. (2005). The action research dissertation: A guide for students and faculty. London, England: Sage.

Hill, N. E., \& Chao, R. K. (2009). Families schools and the adolescent: Connecting research, policy, and practice. New York, NY: Teachers College Press.

Hill, N. E. \& Taylor, L. C. (2004). Parental school involvement and children's academic achievement. American Psychological Society, 13(4), 161-164.

Hillard, A. (1997). The structure of valid staff development. Journal of Staff Development, 18(2), 28-34. 
Hoover-Dempsey, K. V., Bassler O. C., \& Brissle, J. S. (1987). Parent involvement: Contributions of teacher efficacy, school socioeconomic status, and other school characteristics. American Educational Research Journal, 24(3), 417-435.

Hoover-Dempsey, K. V., Ice, C. I., \& Whitake, M. C. (2009). "We're way past reading together": Why and how parent involvement in adolescence makes sense. In N. E. Hill \& R. K. Chao (Eds.), Families schools and the adolescent: connecting research, policy, and practice (pp. 19-36). New York, NY: Teachers College Press.

Hoover-Dempsey, K. V. \& Sandler, H. M. (1995). Parental involvement in children's education: Why make the difference? Teachers College Record, 97(2), 310-331.

Hoover-Dempsey, K. V., Walker, J. M. T., Sandler, H. M., Whetsel, D., Green, C. L., Wilkins, A. S., \& Closson, K. (2005). Why do parents become involved? Research findings and implications. The Elementary School Journal, 106 (2), 105-130.

Horvat, E. M., Weininger, E. B., \& Lareau, A. (2003). From social ties to social capital: Class differences in the relations between schools and parent networks. American Educational Research Journal, 40, 319-351.

Huckaby, W. O. (1980). Integrating style and purpose in leadership. Educational Leadership, 37(8), 613-616.

Illinois 5Essentials. (2013). Homepage. Retrieved on July 9, 2014, from http://www.isbe.state.il.us/5essentials/default.htm

Illinois State Board of Education. (2009). 2009 Report card definition and sources of data. Retrieved from http://www.isbe.net/research/pdfs/rc09_def.pdf

Illinois State board of Education (2012). The new Illinois learning standards incorporating the common core. Retrieved on June 18, 2012, from http://www.isbe.net/common_core/default.htm

Johnson, S. (1998). Who moved my cheese? New York, NY: G. P. Putnam's Cons.

Jones, N. \& Schneider, B. (2009). Rethinking the role of parenting: Promoting adolescent academic success and emotional well-being. In N. E. Hill \& R. K. Chao (Eds.), Families schools and the adolescent: Connecting research, policy, and practice (pp. 73-90). New York, NY: Teachers College Press.

Joyce, B., \& Showers, B. (2000). Student achievement through staff development $\left(3^{\text {rd }}\right.$ ed.). White Plains, NY: Longman. 
Kemmis, S., \& McTaggart, R. (1988). The action research planner ( $3^{\text {rd }}$ ed.). Australia: Deakin University Press.

Kemmis, S., \& McTaggart, R. (2008). Participatory action research. In N. K. Denzin \& Y. S. Lincoln (Eds.) Strategies of qualitative inquiry (pp. 271-330). Thousand Oaks, CA: Sage Publications.

Kotter, J. P., \& Cohen, D. S. (2002). The heart of change: Real life stories of how people change their organizations. Boston, MA: Harvard Business School Press.

Lincoln-Way Central 5 Essentials. (2013). Homepage. Retrieved on July, 9, 2014, from http://www.illinoisreportcard.com/School.aspx ?source=Environment\&source2 $=5$ EssentialsSurvey\&Schoolid=560992100160001

Lipman-Blumen, J. (1996). Connective leadership: Managing in a changing world. New York: NY: Oxford University Press.

Loughridge, M., \& Tarantino, L. R. (2005). Leading effective secondary school reform. Your guide to strategies that work. Thousand Oaks, CA: Corwin Press.

MacLean, M. S., \& Mohr, M. (1999). Teachers-researchers at work. Berkeley, CA: National Writing Project.

Mapp, K. (2011). Feature: A Conversation with Dr. Karen Mapp, Consultant on Family Engagement. Retrieved on June 20, 2012, from http://www.ed.gov/edblogs/oii/2011/09/feature-a-conversation-with-dr-karenmapp-consultant-on-family-engagement/

Marchant, G. J., Paulson, S. E., \& Rothlisberg, B. A. (2001). Relations of middle schoolstudents' perceptions of family and school contexts with academic achievement. Psychol. Schools, 38(6), 505-519.

Marsh, J. A. (2007). Democratic dilemmas: Joint work, education politics, and community. Albany, NY: State University of New York Press.

Marshall, C., \& Rossman, G. B. (2006). Designing qualitative research. Thousand Oaks, California: Sage Publications.

Marzano, R. J. (2003). What works in schools: Translating research into action. Alexandria, VA: Association for supervision and curriculum development.

Marzano, R. J. (2007). The art and science of teaching: A comprehensive framework for effective instruction. Alexandria, VA: Association for Supervision and Curriculum Development.

Maslow, A. H. (1943). A theory of human motivation. Psychological Review 50(4), 370396. 
McMillan, J. H. (2007). Formative classroom assessment: The key to improving studentachievement. In J. H. McMillan (Ed.), Formative Classroom Assessment: Theory Into Practice (pp. 1-7). New York: Teachers College Press.

McMillan, J. H., \& Hearn, J. (2008). Student self-assessment: The key to stronger student motivation and higher achievement. Educational Horizons, Fall, 40-49.

Merriam, S. B. (2009). Qualitative research: A guide to design and implementation. San Francisco, CA: Jossey Bass.

Mu, M., \& Childs, R. (2005). What parents know and believe about large scale assessments. Canadian Journals of Educational Administration and Policy, 37, $1-24$.

Mullen, C. A. (2010). $21^{\text {st }}$ century priorities for leadership education and prospectrive school leaders. Scholar-Practitioner Quarterly, 4(4), 331-333.

National Commission on Excellence in Education. (1983). A nation at risk: The imperative for educational reform. Retrieved on June 19, 2012, from http://teachertenure.procon.org/sourcefiles/a-nation-at-risk-tenure-april-1983.pdf

No Child Left Behind Act. (2001). Retrieved June 15, 2012, from U.S. Department of Education Web site: http://www.ed.gov/policy/.

O'Connor, K. (2011). A repair kit for grading: 15 fixes for broken grades. Portland, OR: Educational Testing Service.

Paine, S. L. (2008). Classroom assessment for literacy: A journey to assessment literacy. Retrieved on June 22, 2012, from http://wvde.state.wv.us/prinperspective/reflectionsdocs $\% 20 \% 20 \mathrm{PDF} / 21 \% 20 \mathrm{Cent}$ ury\%20Assessment\%20Brochure\%20Single\%20Pages.pdf

Partnership for $21^{\text {st }}$ Century Skills. (2009). P21 definitions. Retrieved on June 21, 2012, from http://p21.org/storage/documents/P21_Framework_Definitions.pdf

Patton, M. Q. (1987). How to use qualitative methods in evaluation. Newbury Park, CA: Sage.

Patton. M. Q. (2002). Qualitative evaluation and research methods. Newbury Park, CA: SAGE.

Pink, D. H. (2009). Drive: The surprising truth about what motivates us. New York, New York: Riverhead Books.

Popham, W. J. (2006). Assessment for learning: An endangered species. Educational Leadership, February, 82-83. 
Popham, W. J. (2007). Instructional insensitivity of tests: Accountability's dire drawback. The Phi Delta Kappan, 89 (2), 146-150.

Popham, W. J. (2008). Transformative Assessment. Alexandria, VA: Association for Supervision and Curriculum Development.

Prestine, N. A., \& McGreal, T. L. (1997). Fragile changes, sturdy lives: Implementing authentic assessment in schools. Educational Administration Quarterly, 33(3), 371-400.

Ravitch, D. (2010). The death and life of the great American school system: How testing and choices are undermining education. New York, NY: Basic Books.

Redding, S., Langdon, J., Meyer, J. \& Sheley, P. (2004). The effects of comprehensive parent engagement on student learning outcomes. Harvard Family Research Project retrieved on June 19, 2012, from http://www.adi.org/solidfoundation/resources/Harvard.pdf

Reeves, D. (2007). Ahead of the curve: The power of assessment to transform teaching and learning. Bloomington, IN: Solution Tree Press.

Rolheiser, C., \&. Ross, J. A (2001). "Student self-evaluation: What research says and what practice shows." Retrieved July 15, 2014, from http://blogs.orchardview.org/perreault/files/2013/11/Student-Self-Evaluationarticle.pdf

Rost, J. C. (1993). Leadership development in the new millennium. Journal of Leadership and Organizational Studies 1(1), 92-110.

Schmoker, M. (2004). High school as it could be: From cacophony to continuous improvement. SEDL letter, 3-7.

Senge, P. (2000), Schools that learn: A fifth discipline field book for educators, parents, and everyone who cares about education. New York, NY: Doubleday.

Shepard, L. A. (2000). The role of assessment in a learning culture. Educational Researcher, 29(7), 4-14.

Shepard L. A., \& Bliem, C. L. (1995) Parents' thinking about standardized tests and performance assessments. Educational Researcher, 24 (8), 25-32.

Silver Strong \& Associates. (2011). The strategic teacher: Better instruction, deeper learning, higher achievement. Retrieved on June 16, 2012, from http://www.thoughtfulclassroom.com/PDFs/TheStrategic TeacherBetterInstruction.pdf 
Sitzmann, T., Ely, K., Brown, K. G., \& Bauer, K. N. (2010). Self-assessment of knowledge: A cognitive learning or affective measure? Academy of Management Learning \& Education, 9 (2), 169-191.

Smit, A. G. \& Liebenberg, L. ( 2003). Understanding the dynamics of parental involvement in schooling within the poverty context. South African Journal of Education, 23(1), 1-5.

Smith. H. (2011). Interactive report card. Retrieved on June 6, 2012, from http://iirc.niu.edu/District.aspx?source=District_Profile\&districtID=56099210016 \&level=D

Sparks, D., \& Loucks-Horsley, S. (1989). Five models of staff development. Journal of Staff Development, 10(4), 40-57.

Spillane, J. P. (2005). Distributed leadership. The Educational Forum, 69(2), 143-150

Spillane, J. P., Halverson, R., \& Diamond, J. B. (2001). Investigating school leadership practice: A distributed perspective. Educational Researcher, 30(3), 23-28.

Spring, J. (2005). Conflict of interests: the politics of American education ( $5^{\text {th }}$ ed.). New York, NY: McGraw Hill.

Steinberg, L., Lamborn, S. D., Dornbusch, S. M., \& Darling, N. (1992). Impact of parenting practices on adolescent achievement: Authoritative parenting, school involvement, and encouragement to succeed. Child Development, 63, 1266-1281.

Stiggins, R. (1999). Assessment, student confidence, and school success. Phi Delta Kappan, November, 191-198.

Stiggins, R. (2002). Assessment crisis: The absence of assessment for learning. Phi Delta Kappan, June, 758-765.

Stiggins, R. (2005a). Assessment for learning defined. ETS /Assessment Training Institute's International Conference. Portland OR, September.

Stiggins, R. (2005b). From formative assessment to assessment FOR learning: A path to success in standards-based schools. Phi Delta Kappan, 87(4), 324-328.

Stiggins, R. (2007). Assessment for learning. In Reeves, D. (Ed.). Ahead of the curve: The power of assessment to transform teaching and learning (pp. 58-78). Bloomington, IN: Solution Tree Press.

Stiggins, R., Arter, J., Chappuis, J., \& Chappuis, S. (2004). Classroom assessment for student Learning: Doing it right-using it well. Portland, OR: Assessment Training Institute. 
Stiggins, R. \& DuFour, R. (2009). Maximizing the power of formative assessment. Phi Delta Kappan, 90(9), 640-644.

Stiggins, R., \& Popham, W. J. (2008). Assessing students' affect related to assessment for learning. Council of Chief State School Officers, PAPER

Strauss, A., \& Corbin, J. (1998). Basics of qualitative research techniques and procedures for developing grounded theory ( $2^{\text {nd }}$ ed.). Sage Publications: London.

Suazo deCastro, B., \& Catsambis, S. (2009). Parents still matter: parental links to the behaviors and future outlook of high school seniors. In N. E. Hill \& R. K. Chao (Eds.), Families schools and the adolescent: connecting research, policy, and practice (pp. 91-109). New York, NY: Teachers College Press.

Susman, G. I., \& Evered, R. D. (1978). An assessment of the scientific merits of action research. Administrative Science Quarterly, 23(4), 582-603.

United States Census Bureau. (2010). Lincoln-Way community fact finder [Data File] Retrieved on June 7, 2012, from http://factfinder2.census.gov/faces/nav/jsf/pages/searchresults.xhtml

Warren, M. R., Hong, S., Rubin, C. H., \& Uy, P. S. (2009). Beyond the bake sale: A community-based relational approach to parent engagement in schools. Teacher College Record, 11(9), 2209-2254.

Weaver, S. M. (2007). Cultivating connections with parents. The Clearing House, 81(1), 5-7.

Webb, M. \& Jones, J. (2009). Exploring tensions in developing assessment for learning. Assessment in Education: Principles, Policy, \& Practice, 16(2), 165-184.

Weiss, H. B., Lopez, M. E., \& Rosenberg, H. (2010). Beyond random acts: Family, school, and community engagement as an integral part of educational reform. Harvard Family Research Project.

Whitehead, J. (1989). Creating a living educational theory from questions of the kind, "How do I improve my practice?". Cambridge Journal of Education, 19(1), 4152.

Whitehead, J. (1998). Educational action researchers creating their own educational theories. Paper presented at the Annual Meeting of American Educational Research Association. San Diego, CA on 3/14/98.

Whitehead, J. (2008). Using a living theory methodology in improving practice and generating educational knowledge in living theories. Educational Journal of Living Theories, 1(1), 103-126. 
Whitehead, J. (2010). As an educator and educational researcher, how do I improve what I am doing and contribute to educational theories that carry hope for the future of humanity? Retrieved from: http://digitalcommons.nl.edu/ie/vol1/iss2/2

Wiggins, G. (1998). Educative Assessment. Designing assessments to inform and improve student performance. San Francisco, CA: Jossey-Bass Inc.

Wiggins, G. (2001). Understanding by design. Retrieved on June 16, 2012, from http://www.thoughtfulclassroom.com/PDFs/TheStrategicTeacherBetterInstruction.pdf

Wiliam, D. (2007). Content then process: Teacher learning communities in the service of formative assessment. In D. Reeves (Ed.), Ahead of the curve: The power of assessment to transform teaching and learning (pp. 79-101). Bloomington, IN, Solution Tree Press.

Wormeli, R. (2006). Fair isn't always equal: Assessing \& grading in the differentiated classroom. Portland, OR: Stenhouse.

Yamamoto, Y. \& Holloway S. D. (2010). Parental expectations and children's academic performance in sociocultural context. Educational Psychology Review, 22, 189214. 
APPENDIX A

SURVEYS 


\section{Asset Program Survey Questions}

\section{Read each statement and mark A for true/yes or B for false/no. \\ Preface all statements with "Overall" or "Generally speaking"}

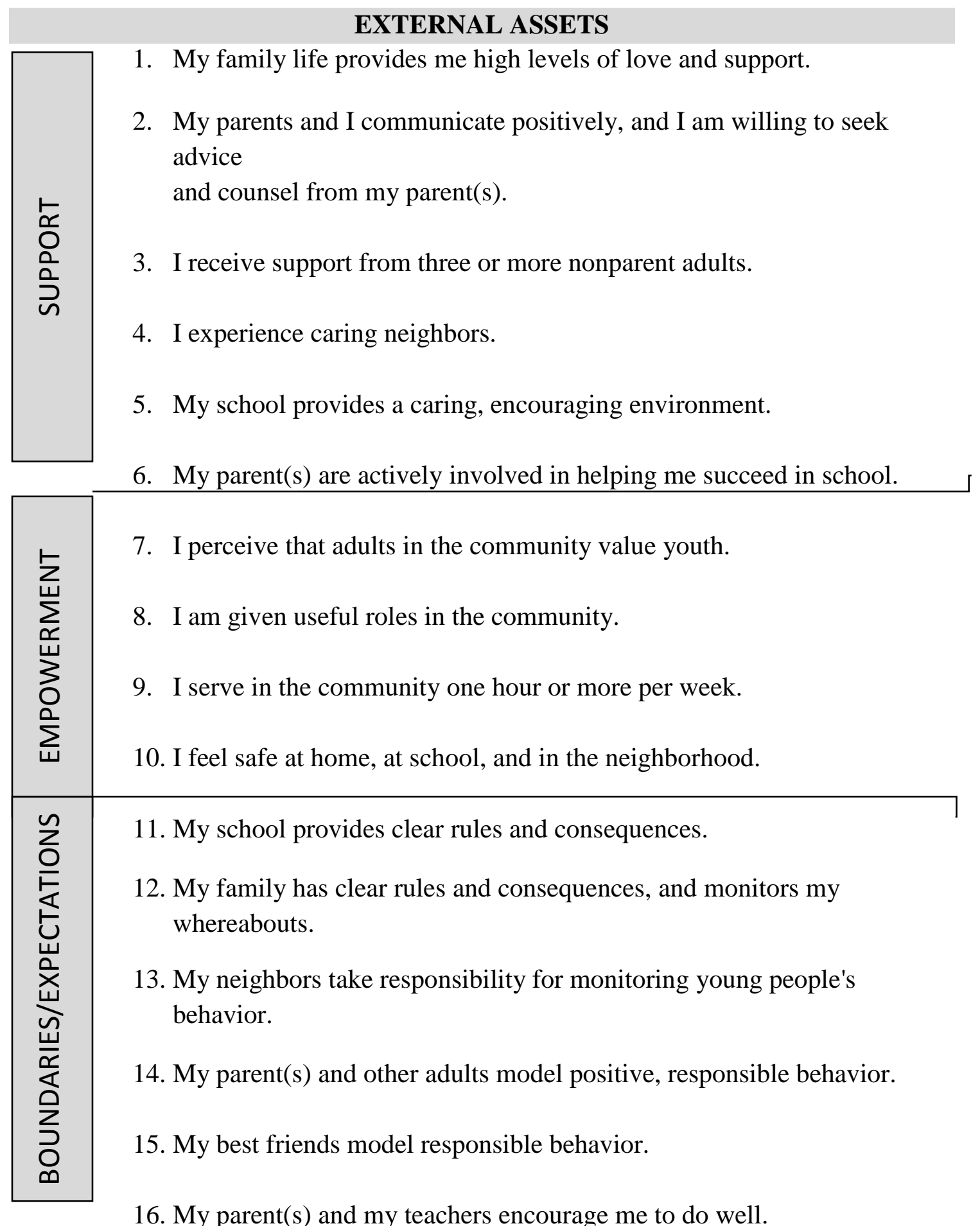




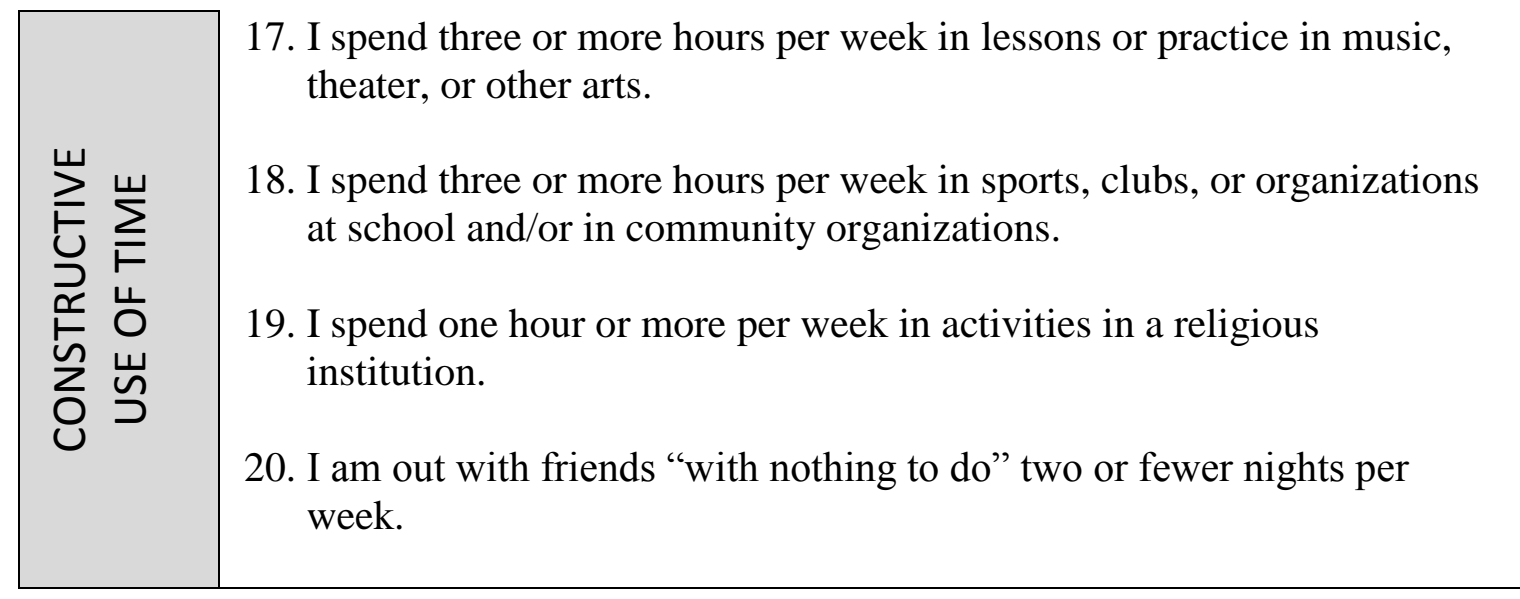

\section{INTERNAL ASSETS}

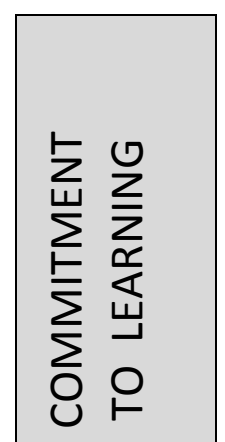

21. I am motivated to do well in school.

22. I am actively engaged in learning.

23. I do at least one hour of homework every school day.

24. I care about my school.

25. I read for pleasure three or more hours per week.

\begin{tabular}{|l|l} 
26. I place high value on helping other people. \\
$\begin{array}{l}\text { 27. I place high value on promoting equality and reducing hunger and } \\
\text { poverty. }\end{array}$ \\
28. I act on my convictions and stand up for my beliefs. \\
$\begin{array}{l}\text { 29. I tell the truth even when it is not easy. } \\
\text { 30. I accept and take personal responsibility. } \\
\text { 31. I believe it is important not to be sexually active or to use alcohol or } \\
\text { other drugs. }\end{array}$
\end{tabular}


32. I know how to plan ahead and make choices.

$\backsim$

33. I have empathy, sensitivity, and friendship skills.

质 2 34. I have knowledge of and comfort with people of different cultural, 임

约

35. I can resist negative peer pressure and dangerous situations.

36. I seek to resolve conflict nonviolently.

\begin{tabular}{|c|c|}
\hline & 37. I have control over "things that happen to me." \\
\hline 亘 & 38. I have high self-esteem. \\
\hline 음 & 39. I believe that "my life has a purpose." \\
\hline & 40. I am optimistic about my personal future. \\
\hline
\end{tabular}




\section{Survey Questions}

Thank you for attending tonight's presentation on final exam review and assessments. Please take a few moments to reflect on the presentation on assessment and gauge your response as strongly disagree (1), disagree (2), neutral (3), agree (4), and strongly agree (5). The information provided will be summarized within a formal research study conducted by Sarah Highfill.

\begin{tabular}{|c|c|c|c|c|c|}
\hline & $\begin{array}{l}\text { strongly } \\
\text { disagree }\end{array}$ & disagree & neutral & agree & $\begin{array}{c}\text { strongly } \\
\text { agree }\end{array}$ \\
\hline $\begin{array}{l}\text { 1. Before tonight, I knew the } \\
\text { difference between } \\
\text { formative and summative } \\
\text { assessment. }\end{array}$ & 1 & 2 & 3 & 4 & 5 \\
\hline $\begin{array}{l}\text { 2. After the presentation, I } \\
\text { know the difference } \\
\text { between formative and } \\
\text { summative assessment. }\end{array}$ & 1 & 2 & 3 & 4 & 5 \\
\hline $\begin{array}{l}\text { While studying for finals, } \\
\text { students must assess their } \\
\text { own strengths and } \\
\text { weakness of the course } \\
\text { objectives. }\end{array}$ & 1 & 2 & 3 & 4 & 5 \\
\hline $\begin{array}{l}\text { 4. I feel comfortable with } \\
\text { supporting Assessment for } \\
\text { Learning (A4L) at home. }\end{array}$ & 1 & 2 & 3 & 4 & 5 \\
\hline $\begin{array}{l}\text { 5. I would like to continue to } \\
\text { discuss assessment in a } \\
\text { focus group. }\end{array}$ & 1 & 2 & 3 & 4 & 5 \\
\hline
\end{tabular}

\section{Please Detach}

If you choose agree or strongly agree to question 5, please provide your name and email address for Sarah Highfill to contact you in regards to a focus group conversation on assessment in the future. Please tear off this bottom section of the survey as you return your responses to the questions above. Thank you for your interest!

Name:

Email address: 
2013 5Essentials Core Student Survey Questions

\begin{tabular}{|c|c|c|c|c|c|}
\hline \multicolumn{6}{|c|}{ 1) How safe do you feel: } \\
\hline & & Not safe & $\begin{array}{l}\text { Somewhat } \\
\text { safe }\end{array}$ & Mostly safe & Very safe \\
\hline a) & $\begin{array}{l}\text { In the hallways and } \\
\text { bathrooms of the school. }\end{array}$ & & & & \\
\hline b) & Outside around the school. & & & & \\
\hline & $\begin{array}{l}\text { Traveling between home } \\
\text { and school }\end{array}$ & & & & \\
\hline & In your classes & & & & \\
\hline
\end{tabular}

\begin{tabular}{|l|l|l|l|l|}
\hline \multicolumn{2}{|c|}{ 2) How much do you agree with the following: } \\
\hline & $\begin{array}{l}\text { Strongly } \\
\text { disagree }\end{array}$ & Disagree & Agree & $\begin{array}{l}\text { Strongly } \\
\text { Agree }\end{array}$ \\
\hline a) & $\begin{array}{l}\text { When my teachers tell } \\
\text { me not to do something, I } \\
\text { know they have a good } \\
\text { reason. }\end{array}$ & & & \\
\hline b) $\begin{array}{l}\text { I feel safe and } \\
\text { comfortable with my } \\
\text { teachers at this school. }\end{array}$ & & & & \\
\hline c) $\begin{array}{l}\text { My teachers keep their } \\
\text { promises. }\end{array}$ & & & & \\
\hline d) & $\begin{array}{l}\text { My teachers will listen to } \\
\text { students' ideas. }\end{array}$ & & & \\
\hline e) $\begin{array}{l}\text { My teachers treat me } \\
\text { with respect. }\end{array}$ & & & & \\
\hline
\end{tabular}

(Grades 6-8; Target = English or Math)

\begin{tabular}{|l|l|l|l|l|l|l|l|}
\hline \multicolumn{2}{|c|}{ 3) How many of the students in your [TARGET] class: } \\
\hline & None & A few & Some & $\begin{array}{l}\text { About } \\
\text { half }\end{array}$ & Most & All \\
\hline $\begin{array}{l}\text { a) } \\
\text { Feel it is important to } \\
\text { come to school every } \\
\text { day. }\end{array}$ & & & & & & \\
\hline b) & $\begin{array}{l}\text { Feel it is important to } \\
\text { pay attention in class. }\end{array}$ & & & & & & \\
\hline c) & $\begin{array}{l}\text { Think doing homework } \\
\text { is important. }\end{array}$ & & & & & & \\
\hline d) & $\begin{array}{l}\text { Try hard to get good } \\
\text { grades. }\end{array}$ & & & & & & \\
\hline
\end{tabular}




\begin{tabular}{|l|l|l|l|l|}
\hline \multicolumn{2}{|c|}{ 4) How much do you agree with the following statements about your [TARGET] } \\
class:
\end{tabular}

\section{5) In my [TARGET] class, my teacher:}

\begin{tabular}{|l|l|l|l|l|}
\hline & $\begin{array}{l}\text { Strongly } \\
\text { disagree }\end{array}$ & Disagree & Agree & $\begin{array}{l}\text { Strongly } \\
\text { Agree }\end{array}$ \\
\hline a) & $\begin{array}{l}\text { Expects everyone to } \\
\text { work hard. }\end{array}$ & & & \\
\hline b) $\begin{array}{l}\text { Expects me to do my best } \\
\text { all the time. }\end{array}$ & & & & \\
\hline c) $\begin{array}{l}\text { Wants us to become } \\
\text { better thinkers, not just } \\
\text { memorize things. }\end{array}$ & & & & \\
\hline
\end{tabular}

\section{6) In your [TARGET] class, how often:}

\begin{tabular}{|c|c|c|c|c|c|}
\hline & & Never & $\begin{array}{l}\text { Once in a } \\
\text { while }\end{array}$ & $\begin{array}{l}\text { Most of the } \\
\text { time }\end{array}$ & $\begin{array}{l}\text { All of the } \\
\text { time }\end{array}$ \\
\hline a) & Are you challenged? & & & & \\
\hline & $\begin{array}{l}\text { Do you have to work hard } \\
\text { to do well? }\end{array}$ & & & & \\
\hline c) & $\begin{array}{l}\text { Does the teacher ask } \\
\text { difficult questions on tests? }\end{array}$ & & & & \\
\hline & $\begin{array}{l}\text { Does the teacher ask } \\
\text { difficult questions in class? }\end{array}$ & & & & \\
\hline
\end{tabular}

\section{7) How much do you agree with the following statements about your [TARGET]} class:

\begin{tabular}{|c|c|c|c|c|c|}
\hline & & $\begin{array}{l}\text { Strongly } \\
\text { disagree }\end{array}$ & Disagree & Agree & $\begin{array}{l}\text { Strongly } \\
\text { Agree }\end{array}$ \\
\hline & $\begin{array}{l}\text { I learn a lot from } \\
\text { feedback on my work }\end{array}$ & & & & \\
\hline b) & $\begin{array}{l}\text { It's clear to me what I } \\
\text { need to do to get a good } \\
\text { grade. }\end{array}$ & & & & \\
\hline c) & $\begin{array}{l}\text { The work we do in class } \\
\text { is good preparation for } \\
\text { the test. }\end{array}$ & & & & \\
\hline d) & $\begin{array}{l}\text { The homework } \\
\text { assignments help me to } \\
\text { learn the course material. }\end{array}$ & & & & \\
\hline e) & $\begin{array}{l}\text { I know what my teacher } \\
\text { wants me to learn in this } \\
\text { class. }\end{array}$ & & & & \\
\hline
\end{tabular}




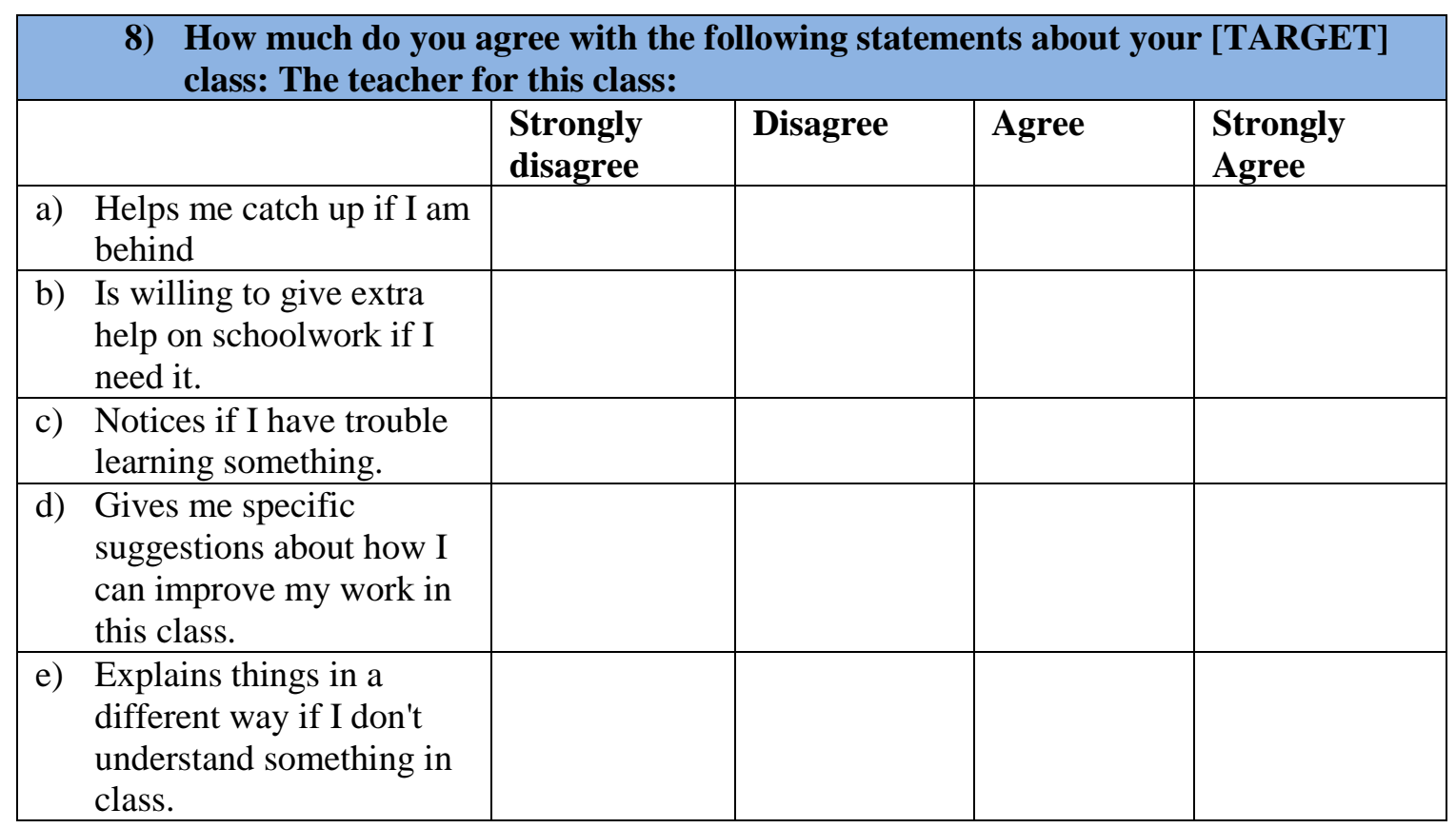

(Grades 9-12)

\begin{tabular}{|c|c|c|c|c|c|}
\hline & & $\begin{array}{l}\text { Strongly } \\
\text { disagree }\end{array}$ & Disagree & Agree & $\begin{array}{l}\text { Strongly } \\
\text { Agree }\end{array}$ \\
\hline & $\begin{array}{l}\text { Teachers make sure that } \\
\text { all students are planning } \\
\text { for life after graduation. }\end{array}$ & & & & \\
\hline b) & $\begin{array}{l}\text { Teachers work hard to } \\
\text { make sure that all } \\
\text { students are learning }\end{array}$ & & & & \\
\hline c) & $\begin{array}{l}\text { High school is seen as } \\
\text { preparation for the future. }\end{array}$ & & & & \\
\hline d) & $\begin{array}{l}\text { All students are } \\
\text { encouraged to go to } \\
\text { college. }\end{array}$ & & & & \\
\hline e) & $\begin{array}{l}\text { Teachers pay attention to } \\
\text { all students, not just the } \\
\text { top students. }\end{array}$ & & & & \\
\hline f) & $\begin{array}{l}\text { Teachers work hard to } \\
\text { make sure that students } \\
\text { stay in school. }\end{array}$ & & & & \\
\hline
\end{tabular}


(All Students)

\begin{tabular}{|c|c|c|c|c|c|c|}
\hline & \multicolumn{6}{|c|}{$\begin{array}{l}\text { 10) In your ENGLISH/READING/LITERATURE class this year, how often do you } \\
\text { do the following: }\end{array}$} \\
\hline & & Never & $\begin{array}{l}\text { Once or } \\
\text { twice a } \\
\text { semester }\end{array}$ & $\begin{array}{l}\text { Once or } \\
\text { twice a } \\
\text { month }\end{array}$ & $\begin{array}{l}\text { Once or } \\
\text { twice a } \\
\text { week }\end{array}$ & $\begin{array}{l}\text { Almost } \\
\text { every day }\end{array}$ \\
\hline & $\begin{array}{l}\text { Debate the meaning of a } \\
\text { reading. }\end{array}$ & & & & & \\
\hline b) & $\begin{array}{l}\text { Discuss connections } \\
\text { between a reading and } \\
\text { real life people or } \\
\text { situations. }\end{array}$ & & & & & \\
\hline c) & $\begin{array}{l}\text { Discuss how culture, } \\
\text { time, or place affects an } \\
\text { author's writing. }\end{array}$ & & & & & \\
\hline d) & $\begin{array}{l}\text { Improve a piece of } \\
\text { writing as a class or with } \\
\text { partners. }\end{array}$ & & & & & \\
\hline e) & $\begin{array}{l}\text { Rewrite a paper or essay } \\
\text { in response to } \\
\text { comments. }\end{array}$ & & & & & \\
\hline f) & $\begin{array}{l}\text { (Grades 9-12 Only) } \\
\text { Explain how writers use } \\
\text { tools like symbolism and } \\
\text { metaphor to } \\
\text { communicate meaning }\end{array}$ & & & & & \\
\hline
\end{tabular}

\section{1) In your MATH class this year, how often do you do the following:}

\begin{tabular}{|l|l|l|l|l|l|}
\hline & Never & $\begin{array}{l}\text { Once or } \\
\text { twice a } \\
\text { semester }\end{array}$ & $\begin{array}{l}\text { Once or } \\
\text { twice a } \\
\text { month }\end{array}$ & $\begin{array}{l}\text { Once or } \\
\text { twice a } \\
\text { week }\end{array}$ & $\begin{array}{l}\text { Almost } \\
\text { every day }\end{array}$ \\
\hline a) & $\begin{array}{l}\text { Apply math to situations } \\
\text { in life outside of school. }\end{array}$ & & & & \\
\hline b) $\begin{array}{l}\text { Discuss possible } \\
\text { solutions to problems } \\
\text { with other students. }\end{array}$ & & & & & \\
\hline c) & $\begin{array}{l}\text { Explain how you solved } \\
\text { a problem to the class. }\end{array}$ & & & & \\
\hline d) $\begin{array}{l}\text { Write a few sentences to } \\
\text { explain how you solved } \\
\text { a math problem. }\end{array}$ & & & & & \\
\hline e) $\begin{array}{l}\text { Write a math problem } \\
\text { for other students to } \\
\text { solve. }\end{array}$ & & & & & \\
\hline f) $\begin{array}{l}\text { (Grades 9-12 Only) } \\
\text { Solve a problem with } \\
\text { multiple steps that takes } \\
\text { more than 20 minutes. }\end{array}$ & & & & & \\
\hline
\end{tabular}




\begin{tabular}{|c|c|c|c|c|c|}
\hline \multicolumn{6}{|c|}{$\begin{array}{l}\text { 12) How much do you agree with the following statements about the community in } \\
\text { which you live: }\end{array}$} \\
\hline & & $\begin{array}{l}\text { Strongly } \\
\text { disagree }\end{array}$ & Disagree & Agree & $\begin{array}{l}\text { Strongly } \\
\text { Agree }\end{array}$ \\
\hline a) & $\begin{array}{l}\text { Adults in this } \\
\text { neighborhood know who } \\
\text { the local children are }\end{array}$ & & & & \\
\hline b) & $\begin{array}{l}\text { During the day, it is safe } \\
\text { for children to play in the } \\
\text { local park or playground. }\end{array}$ & & & & \\
\hline c) & $\begin{array}{l}\text { People in this } \\
\text { neighborhood can be } \\
\text { trusted. }\end{array}$ & & & & \\
\hline & $\begin{array}{l}\text { There are adults in this } \\
\text { neighborhood that } \\
\text { children can look up to. }\end{array}$ & & & & \\
\hline e) & $\begin{array}{l}\text { The equipment and } \\
\text { buildings in the } \\
\text { neighborhood park or } \\
\text { playground are well kept. }\end{array}$ & & & & \\
\hline
\end{tabular}


2013 5Essentials Core Teacher Survey Questions

\begin{tabular}{|c|c|c|c|c|c|}
\hline \multicolumn{6}{|c|}{ 13) How many teachers in this school: } \\
\hline & None & Some & About half & Most & Nearly all \\
\hline $\begin{array}{l}\text { g) Help maintain discipline in } \\
\text { the entire school, not just } \\
\text { their classroom. }\end{array}$ & & & & & \\
\hline $\begin{array}{l}\text { h) Take responsibility for } \\
\text { improving the school. }\end{array}$ & & & & & \\
\hline $\begin{array}{l}\text { i) Feel responsible to help } \\
\text { each other do their best. }\end{array}$ & & & & & \\
\hline $\begin{array}{l}\text { j) Feel responsible that all } \\
\text { students learn. }\end{array}$ & & & & & \\
\hline $\begin{array}{l}\text { k) Feel responsible for } \\
\text { helping students develop } \\
\text { selfcontrol. }\end{array}$ & & & & & \\
\hline $\begin{array}{l}\text { 1) Feel responsible when } \\
\text { students in this school fail. }\end{array}$ & & & & & \\
\hline
\end{tabular}

\begin{tabular}{|c|c|c|c|c|}
\hline \multicolumn{5}{|c|}{$\begin{array}{l}\text { 14) Please mark the extent to which you disagree or agree with each of the following } \\
\text { statements about your school: }\end{array}$} \\
\hline & $\begin{array}{l}\text { Strongly } \\
\text { disagree }\end{array}$ & Disagree & Agree & $\begin{array}{l}\text { Strongly } \\
\text { Agree }\end{array}$ \\
\hline $\begin{array}{l}\text { g) Principal pushes teachers to } \\
\text { communicate regularly } \\
\text { with parents. }\end{array}$ & & & & \\
\hline $\begin{array}{l}\text { h) School staff members } \\
\text { (teachers, counselors, } \\
\text { office staff, etc.) encourage } \\
\text { feedback from parents and } \\
\text { the community }\end{array}$ & & & & \\
\hline $\begin{array}{l}\text { i) Teachers really try to } \\
\text { understand parents' } \\
\text { problems and concerns. }\end{array}$ & & & & \\
\hline $\begin{array}{l}\text { j) Parents are greeted warmly } \\
\text { when they call or visit the } \\
\text { school. }\end{array}$ & & & & \\
\hline $\begin{array}{l}\text { k) Teachers work closely with } \\
\text { parents to meet students' } \\
\text { needs. }\end{array}$ & & & & \\
\hline $\begin{array}{l}\text { 1) School staff members } \\
\text { (teachers, counselors, } \\
\text { office staff, etc.) communi- } \\
\text { cate with parents about } \\
\text { support needed to advance } \\
\text { the school mission. }\end{array}$ & & & & \\
\hline $\begin{array}{l}\text { m) This school regularly } \\
\text { communicates with parents } \\
\text { about how they can help } \\
\text { their children learn. }\end{array}$ & & & & \\
\hline
\end{tabular}


15) How many teachers at this school feel good about parents' support for their work?

\begin{tabular}{l|l|l|l|l|} 
None & Some & About half & Most & Nearly all \\
\hline
\end{tabular}

16) For the students you teach this year, how many of their parents:

\begin{tabular}{|c|c|c|c|c|c|c|}
\hline & & None & Some & About half & Most & All \\
\hline a) & $\begin{array}{l}\text { Support your teaching } \\
\text { efforts. }\end{array}$ & & & & & \\
\hline b) & $\begin{array}{l}\text { Do their best to help their } \\
\text { children learn. }\end{array}$ & & & & & \\
\hline
\end{tabular}

\section{7) To what extent do you feel respected by the parents of your students?}

\begin{tabular}{|l|l|l|l|l|}
\hline & Not at all & A little & Some & $\begin{array}{l}\text { To a great } \\
\text { extent }\end{array}$ \\
\hline
\end{tabular}

\begin{tabular}{|c|c|c|c|c|c|}
\hline \multicolumn{6}{|c|}{ 18) Please mark the extent to which you disagree or agree with each of the following } \\
\hline & & $\begin{array}{l}\text { Strongly } \\
\text { disagree }\end{array}$ & Disagree & Agree & $\begin{array}{l}\text { Strongly } \\
\text { Agree }\end{array}$ \\
\hline & $\begin{array}{l}\text { Teachers and parents } \\
\text { think of each other as } \\
\text { partners in educating } \\
\text { children. }\end{array}$ & & & & \\
\hline & $\begin{array}{l}\text { Staff at this school work } \\
\text { hard to build trusting } \\
\text { relationships with parents. }\end{array}$ & & & & \\
\hline
\end{tabular}

\section{9) To what extent do you feel respected by your principal?}

\begin{tabular}{|l|l|l|l|l|}
\hline \multicolumn{6}{|c|}{ 19) To what extent do you feel respected by your principal? } \\
\hline & Not at all & A little & Some & $\begin{array}{l}\text { To a great } \\
\text { extent }\end{array}$ \\
\hline
\end{tabular}

\section{0) Please mark the extent to which you disagree or agree with each of the following:}

\begin{tabular}{|l|l|l|l|l|}
\hline & $\begin{array}{l}\text { Strongly } \\
\text { disagree }\end{array}$ & Disagree & Agree & $\begin{array}{l}\text { Strongly } \\
\text { Agree }\end{array}$ \\
\hline a) & $\begin{array}{l}\text { The principal has } \\
\text { confidence in the } \\
\text { expertise of the teachers. }\end{array}$ & & & \\
\hline b) $\begin{array}{l}\text { I trust the principal at his } \\
\text { or her word. }\end{array}$ & & & & \\
\hline c) $\begin{array}{l}\text { It's OK in this school to } \\
\text { discuss feelings, worries, } \\
\text { and frustrations with the } \\
\text { principal. }\end{array}$ & & & & \\
\hline d) $\begin{array}{l}\text { The principal takes a } \\
\text { personal interest in the } \\
\text { professional development } \\
\text { of teachers. }\end{array}$ & & & & \\
\hline
\end{tabular}




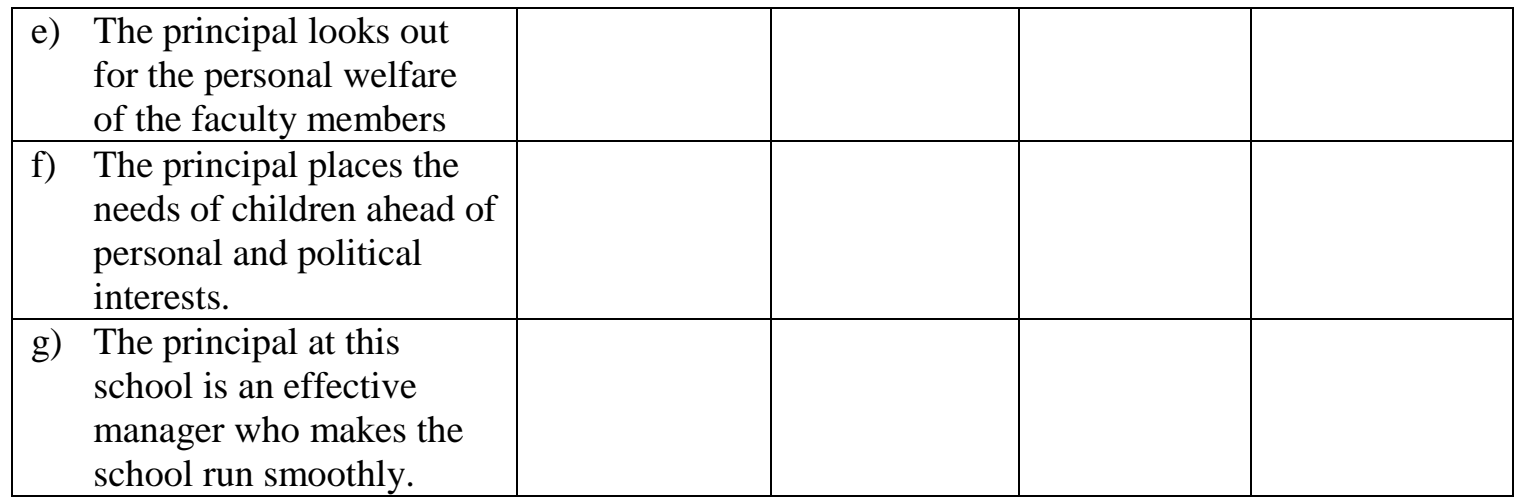

\section{1) To what extent do you feel respected by other teachers?}

\begin{tabular}{|l|l|l|l|l|}
\hline & Not at all & A little & Some & $\begin{array}{l}\text { To a great } \\
\text { extent }\end{array}$ \\
\hline
\end{tabular}

\section{2) Please mark the extent to which you disagree or agree with each of the following:}

\begin{tabular}{|l|l|l|l|l|}
\hline & $\begin{array}{l}\text { Strongly } \\
\text { disagree }\end{array}$ & Disagree & Agree & $\begin{array}{l}\text { Strongly } \\
\text { Agree }\end{array}$ \\
\hline a) $\begin{array}{l}\text { Teachers in this school } \\
\text { trust each other. }\end{array}$ & & & & \\
\hline b) $\begin{array}{l}\text { It's OK in this school to } \\
\text { discuss feelings, worries, } \\
\text { and frustrations with other } \\
\text { teachers }\end{array}$ & & & & \\
\hline c) $\begin{array}{l}\text { Teachers respect other } \\
\text { teachers who take the lead } \\
\text { in school improvement } \\
\text { efforts. }\end{array}$ & & & & \\
\hline d) $\begin{array}{l}\text { Teachers at this school } \\
\text { respect those colleagues } \\
\text { who are experts at their } \\
\text { craft. }\end{array}$ & & & & \\
\hline
\end{tabular}

\section{3) Please mark the extent to which you disagree or agree with each of the following:}

\begin{tabular}{|c|c|c|c|c|}
\hline & $\begin{array}{l}\text { Strongly } \\
\text { disagree }\end{array}$ & Disagree & Agree & $\begin{array}{l}\text { Strongly } \\
\text { Agree }\end{array}$ \\
\hline $\begin{array}{l}\text { a) I usually look forward to } \\
\text { each working day at this } \\
\text { school. }\end{array}$ & & & & \\
\hline $\begin{array}{l}\text { b) I wouldn't want to work } \\
\text { in any other school. }\end{array}$ & & & & \\
\hline c) I feel loyal to this school. & & & & \\
\hline $\begin{array}{l}\text { d) I would recommend this } \\
\text { school to parents seeking } \\
\text { a place for their child. }\end{array}$ & & & & \\
\hline
\end{tabular}


24) For the students you teach this year, how many of their parents:

a) Attended parent-teacher conferences when you requested them.

b) Volunteered time to support the school (e.g., volunteer in classrooms, help with school-wide events, etc.)

c) Contacted me about their child's performance.

d) Respond to my suggestions for helping their child.

25) Please mark the extent to which you disagree or agree with each of the following. The principal at this school:

a) Makes clear to the staff his or her expectations for meeting instructional goals.

b) Communicates a clear vision for our school.

c) Understands how children learn.

d) Sets high standards for student learning.

e) Presses teachers to implement what they have learned in professional development.

f) Carefully tracks student academic progress.

g) Knows what's going on in my classroom.

h) Participates in instructional planning with teams of teachers.

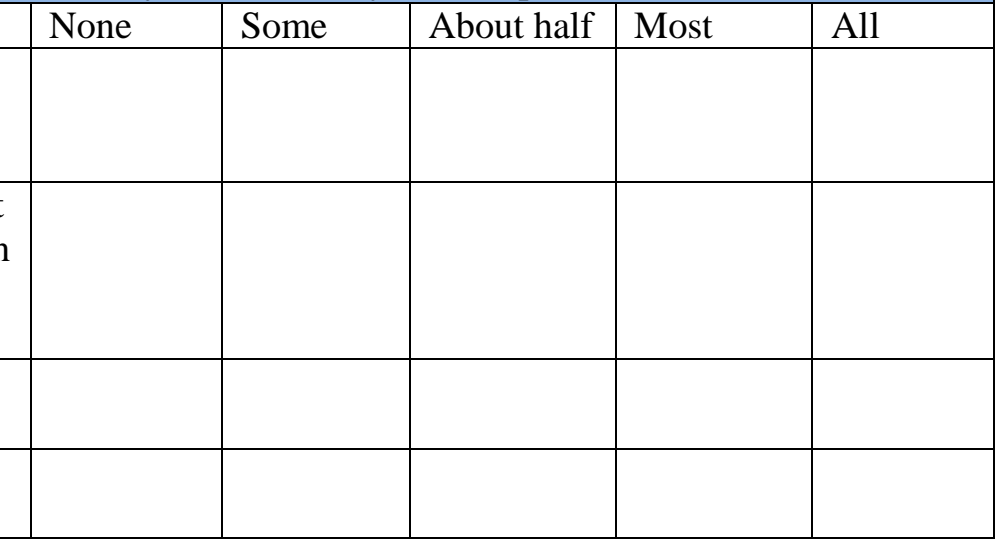

26) How much influence do teachers have over school policy in each of the areas below?

\begin{tabular}{|l|l|l|l|l|}
\hline & Not at all & A little & Some & $\begin{array}{l}\text { To a great } \\
\text { extent }\end{array}$ \\
\hline a) $\begin{array}{l}\text { Determining books and other } \\
\text { instructional materials used in } \\
\text { classrooms. }\end{array}$ & & & & \\
\hline b) $\begin{array}{l}\text { Determining books and other } \\
\text { instructional materials used in } \\
\text { classrooms. }\end{array}$ & & & & \\
\hline
\end{tabular}




\begin{tabular}{|l|l|l|l|l|}
\hline c) $\begin{array}{l}\text { Determining the content of } \\
\text { in-service programs }\end{array}$ & & & & \\
\hline d) $\begin{array}{l}\text { Setting standards for student } \\
\text { behavior. }\end{array}$ & & & & \\
\hline
\end{tabular}

\section{7) To what extent do you disagree or agree with the following:}

\begin{tabular}{|c|c|c|c|c|c|}
\hline & & $\begin{array}{l}\text { Strongly } \\
\text { disagree }\end{array}$ & Disagree & Agree & $\begin{array}{l}\text { Strongly } \\
\text { Agree }\end{array}$ \\
\hline a) & $\begin{array}{l}\text { Once we start a new } \\
\text { program, we follow up to } \\
\text { make sure that it's working }\end{array}$ & & & & \\
\hline b) & $\begin{array}{l}\text { We have so many different } \\
\text { programs in this school } \\
\text { that I can't keep track of } \\
\text { them all. }\end{array}$ & & & & \\
\hline c) & $\begin{array}{l}\text { Many special programs } \\
\text { come and go at this school. }\end{array}$ & & & & \\
\hline d) & $\begin{array}{l}\text { Curriculum, instruction, } \\
\text { and learning materials are } \\
\text { well coordinated across the } \\
\text { different grade levels at } \\
\text { this school. }\end{array}$ & & & & \\
\hline e) & $\begin{array}{l}\text { There is consistency in } \\
\text { curriculum, instruction, } \\
\text { and learning materials } \\
\text { among teachers in the } \\
\text { same grade level at this } \\
\text { school. }\end{array}$ & & & & \\
\hline
\end{tabular}

\section{8) Overall, my professional development experiences this year have:}

\begin{tabular}{|c|c|c|c|c|c|}
\hline & & $\begin{array}{l}\text { Strongly } \\
\text { disagree }\end{array}$ & Disagree & Agree & $\begin{array}{l}\text { Strongly } \\
\text { Agree }\end{array}$ \\
\hline & $\begin{array}{l}\text { Been sustained and } \\
\text { coherently focused, rather } \\
\text { than short-term and } \\
\text { unrelated. }\end{array}$ & & & & \\
\hline b) & $\begin{array}{l}\text { Included enough time to } \\
\text { think carefully about, try, } \\
\text { and evaluate new ideas }\end{array}$ & & & & \\
\hline c) & $\begin{array}{l}\text { Been closely connected to } \\
\text { my school's improvement } \\
\text { plan. }\end{array}$ & & & & \\
\hline d) & $\begin{array}{l}\text { Included opportunities to } \\
\text { work productively with } \\
\text { colleagues in my school. }\end{array}$ & & & & \\
\hline & $\begin{array}{l}\text { Included opportunities to } \\
\text { work productively with } \\
\text { teachers from other } \\
\text { schools. }\end{array}$ & & & & \\
\hline
\end{tabular}


(Grades 9-12)

\begin{tabular}{|l|l|l|l|l|}
\hline \multicolumn{3}{|c|}{ 29) Please mark the extent to which you disagree or agree with each of the following: } \\
\hline & $\begin{array}{l}\text { Strongly } \\
\text { disagree }\end{array}$ & Disagree & Agree & $\begin{array}{l}\text { Strongly } \\
\text { Agree }\end{array}$ \\
\hline a) & $\begin{array}{l}\text { Teachers expect most } \\
\text { students in this school to } \\
\text { go to college. }\end{array}$ & & & \\
\hline b) & $\begin{array}{l}\text { Teachers at this school } \\
\text { help students plan for } \\
\text { college outside of class } \\
\text { time. }\end{array}$ & & & \\
\hline c) & $\begin{array}{l}\text { The curriculum at this } \\
\text { school is focused on } \\
\text { helping students get ready } \\
\text { for college }\end{array}$ & & & \\
\hline d) $\begin{array}{l}\text { Most of the students in } \\
\text { this school are planning to } \\
\text { go to college. }\end{array}$ & & & & \\
\hline e) & $\begin{array}{l}\text { Teachers in this school } \\
\text { feel that it is a part of } \\
\text { their job to prepare } \\
\text { students to succeed in } \\
\text { college. }\end{array}$ & & & \\
\hline
\end{tabular}

(All Teachers; Target= Primary Subject and Specified Period)

\begin{tabular}{|c|c|c|c|c|c|}
\hline \multicolumn{6}{|c|}{$\begin{array}{l}\text { 30) To what extent do the following characteristics describe discussions that occur in } \\
\text { your [TARGET] or [Self-contained] class: }\end{array}$} \\
\hline & & Never & Rarely & Sometimes & $\begin{array}{l}\text { Almost } \\
\text { Always }\end{array}$ \\
\hline & $\begin{array}{l}\text { Students build on each } \\
\text { other's ideas during } \\
\text { discussion. }\end{array}$ & & & & \\
\hline & $\begin{array}{l}\text { Students use data and text } \\
\text { references to support their } \\
\text { ideas. }\end{array}$ & & & & \\
\hline & $\begin{array}{l}\text { Students show each other } \\
\text { respect. }\end{array}$ & & & & \\
\hline & $\begin{array}{l}\text { Students provide } \\
\text { constructive feedback to } \\
\text { their peers and to me. }\end{array}$ & & & & \\
\hline & $\begin{array}{l}\text { Most students participate } \\
\text { in the discussion at some } \\
\text { point. }\end{array}$ & & & & \\
\hline
\end{tabular}

31) How active are your parent organizations (e.g., PTA, PTO)?

\begin{tabular}{|l|l|l|l|l|}
$\begin{array}{l}\text { We do not have } \\
\text { a parent } \\
\text { organization }\end{array}$ & $\begin{array}{l}\text { Not } \\
\text { active }\end{array}$ & $\begin{array}{l}\text { A little } \\
\text { active }\end{array}$ & $\begin{array}{l}\text { Somewhat } \\
\text { active }\end{array}$ & $\begin{array}{l}\text { Very } \\
\text { active }\end{array}$ \\
\hline
\end{tabular}


32) How much influence do parents have on school improvement plans?

\begin{tabular}{|c|c|c|c|c|c|}
\hline & \begin{tabular}{l|l} 
No in \\
\end{tabular} & fluence & \begin{tabular}{l|l} 
A little & $\vdots$ \\
influence & $\mathrm{i}$ \\
\end{tabular} & $\begin{array}{l}\text { Some } \\
\text { influence }\end{array}$ & $\begin{array}{l}\text { A great deal of } \\
\text { influence }\end{array}$ \\
\hline \multicolumn{6}{|c|}{ 33) To what extent does this school: } \\
\hline & & Not at all & A little & Somewhat & A great deal \\
\hline \multicolumn{2}{|c|}{$\begin{array}{l}\text { a) Involve parents in the } \\
\text { development of programs } \\
\text { aimed at improving stu- } \\
\text { dents' academic outcomes? }\end{array}$} & & & & \\
\hline & $\begin{array}{l}\text { Involve parents in choosing } \\
\text { school curricula? }\end{array}$ & & & & \\
\hline \multicolumn{2}{|c|}{$\begin{array}{l}\text { c) Include parent leaders from } \\
\text { all backgrounds in school } \\
\text { improvement efforts? }\end{array}$} & & & & \\
\hline & $\begin{array}{l}\text { Develop formal networks to } \\
\text { link all families with each } \\
\text { other (for example: sharing } \\
\text { parent directories or provid- } \\
\text { ing a website for parents to } \\
\text { connect with one another)? }\end{array}$ & & & & \\
\hline e) & $\begin{array}{l}\text { Encourage more involved } \\
\text { parents to reach out to less- } \\
\text { involved parents? }\end{array}$ & & & & \\
\hline \multicolumn{6}{|c|}{ 34) How often does this school: } \\
\hline \multicolumn{2}{|r|}{$\mid$} & Never & Once a year & $\begin{array}{l}2-3 \text { times a } \\
\text { year }\end{array}$ & $\begin{array}{l}\text { More than } 3 \\
\text { times a year }\end{array}$ \\
\hline \multicolumn{2}{|c|}{$\begin{array}{l}\text { a) Conduct workshops or } \\
\text { trainings for parents on } \\
\text { student learning? }\end{array}$} & & & & \\
\hline \multicolumn{6}{|c|}{ 35) To what extent does this school: } \\
\hline & & Not at all & A little & Somewhat & A great deal \\
\hline \multicolumn{2}{|c|}{$\begin{array}{l}\text { a) Help connect families to } \\
\text { appropriate community } \\
\text { resources? }\end{array}$} & & & & \\
\hline \multicolumn{2}{|c|}{$\begin{array}{l}\text { b) Work with community } \\
\text { businesses, agencies, and } \\
\text { volunteers to offer after- } \\
\text { school programs for } \\
\text { students? }\end{array}$} & & & & \\
\hline & $\begin{array}{l}\text { Collaborate with outside } \\
\text { organizations, such as } \\
\text { businesses, libraries, parks, } \\
\text { and museums to enhance the } \\
\text { learning environment? }\end{array}$ & & & & \\
\hline \multicolumn{2}{|c|}{$\begin{array}{l}\text { d) Opens its building for use by } \\
\text { the community after school } \\
\text { hours? }\end{array}$} & & & & \\
\hline
\end{tabular}




\section{Focus Group Survey}

Today's Date:

Thank you for attending tonight's focus group. I appreciate your conversation and feedback in regards to my research goals.

Before you leave this evening, please take a moment to answer the following questions. Gauge your response as strongly disagree (1), disagree (2), neutral (3), agree (4), and strongly agree (5).

\begin{tabular}{|c|c|c|c|c|c|}
\hline & $\begin{array}{l}\text { strongly } \\
\text { disagree }\end{array}$ & disagree & neutral & agree & $\begin{array}{c}\text { strongly } \\
\text { agree }\end{array}$ \\
\hline $\begin{array}{l}\text { 1. Before tonight's discussion, I } \\
\text { was mostly unaware of the the } \\
\text { Assessment for Learning } \\
\text { (A4L) concept. }\end{array}$ & 1 & 2 & 3 & 4 & 5 \\
\hline $\begin{array}{l}\text { 2. After tonight's discussion, I } \\
\text { am interested in learning more } \\
\text { about the Assessment for } \\
\text { Learning (A4L) concept. }\end{array}$ & 1 & 2 & 3 & 4 & 5 \\
\hline $\begin{array}{l}\text { 3. After tonight's discussion, I } \\
\text { am aware that the A4L } \\
\text { initiative will impact my } \\
\text { child's learning in high school. }\end{array}$ & 1 & 2 & 3 & 4 & 5 \\
\hline $\begin{array}{l}\text { 4. After tonight's discussion, I } \\
\text { am interested in remaining a } \\
\text { partner in making A4L } \\
\text { successful within the district. }\end{array}$ & 1 & 2 & 3 & 4 & 5 \\
\hline $\begin{array}{l}\text { 5. After tonight's discussion, I } \\
\text { am excited and confident to } \\
\text { talk about the concept with my } \\
\text { child and to note any impact in } \\
\text { his/her study habits. }\end{array}$ & 1 & 2 & 3 & 4 & 5 \\
\hline $\begin{array}{l}\text { 6. After tonight's discussion, I } \\
\text { am excited and confident to } \\
\text { share the A4L concept with } \\
\text { other parents who have not } \\
\text { been a part of these focus } \\
\text { groups. }\end{array}$ & 1 & 2 & 3 & 4 & 5 \\
\hline $\begin{array}{l}\text { 7. After tonight's discussion, I } \\
\text { am excited and confident to } \\
\text { discuss the benefits of A4L } \\
\text { with teachers within the } \\
\text { district. }\end{array}$ & 1 & 2 & 3 & 4 & 5 \\
\hline
\end{tabular}


APPENDIX B

FOCUS GROUP SURVEY 


\section{Focus Group Semi-Structured Protocol}

Good Evening. I am Sarah Highfill, Department Chair for Science at Central High School and Illinois State University graduate student. Thank you for volunteering your time this evening.

This night will be productive and informative, not just for me but also for you. I hope to open the door to high school assessment, its expectations, and how this district can collaborate with parents to find a good solution for student academic success. Assessment for Learning (A4L) is the research based educational trend that is the current buzz word. But what does A4L stand for and how does it correlate to student achievement? Tonight, I will ask questions stemming from highlights of the Assessment for Learning initiative, but more importantly, I will ask questions that require your feedback. Tonight's goal is to work with you, the parents, to promote success of your high school aged child (ren) through conversation and discussion.

I hope you are willing to share your thoughts with me tonight along with this small group as we talk through the components of A4L. If you do not feel comfortable answering any question, your silence is respected. I am audiotaping this session only to reflect upon your answers to formulate a plan that best supports the efforts of $\mathrm{A} 4 \mathrm{~L}$ at home and at school. I would like to begin after you take a minute to review the letter of consent. I can answer any questions that you have at this time.

1. This district promotes an asset program that highlights values to encourage young adults to be respectful, considerate, and accountable for their actions. Typically, the student responses direct the program toward particular discussions and presentations conducted monthly in advisory periods. The students answered honestly with high positive percentages. Within each category, the positive responses tapered off from freshmen to senior year.

1. My parents and I communicate positively and I am willing to seek advice from my parents. $(75 \%, 74 \%, 71 \%, 80 \%)$

2. My parents are actively involved in helping me succeed in school. (84\%, 80\%, 73\%, $75 \%)$

3. My parents encourage me to do well. (95\%, 94\%, 93\%, 96\%)

4. My parents have clear expectation for me about schoolwork. (90\%, 88\%, 84\%, 83\%)

5. I am motivated to do well in school. $(90 \%, 86 \%, 84 \%, 85 \%)$

6. I do at least one hour of homework on most school days. $(75 \%, 71 \%, 63 \%, 40 \%)$

7. I am satisfied with my grades. $(68 \%, 57 \%, 55 \%, 68 \%)$

8. I do my homework and prepare for tests consistently. (69\%, 58\%, 57\%, 58\%)

9. I believe that I will pass all my classes. (94\%, 91\%, 93\%, 94\%)

Percentages provided in the order of freshmen, sophomores, juniors, seniors.

a. Two interesting points of interest were the decline in positive student responses to those questions involving parents and the expectations regarding coursework. What are the specifics of parent encouragement in academics? What advice do students seek from their parents? In your opinion, why is there a decline in positive student responses from freshmen to senior year? 
b. Another comparison that I would like you to take a look at is the relationship between motivation and grade satisfaction. How can students be motivated to do well in school yet the students are not satisfied with their grades? What role can parents play? What suggestions do you have for the school?

c. The least positive results are the satisfaction with grades and completing homework. Where do parents fit in to buffer these less than positive responses?

2. From the choice of verbs below, which five words do you see as the best descriptor for your child's high school assessments?

\begin{tabular}{|l|l|l|l|}
\hline Define & Formulate & Recall & Apply \\
\hline Explain & Contrast & Explain & Infer \\
\hline Support & Predict & Evaluate & Revise \\
\hline Design & Describe & Analyze & Summarize \\
\hline Solve & Trace & Compare & Estimate \\
\hline
\end{tabular}

3. The ACT Company identifies the following verbs as the most used within a high school curriculum and instruction.

\begin{tabular}{|l|l|l|l|}
\hline Trace & Analyze & Infer & Evaluate \\
\hline Formulate & Describe & Summarize & Compare \\
\hline Contrast & Predict & Support & Explain \\
\hline
\end{tabular}

a. Did your answers from Question 3 correlate? If so, why were the high school assessment verbs a good voice for standardized test usage?

b. If your answers did not match, what suggestions can you make to close the gap between what is seen within the district and the expectations of ACT?

4. The Next Generation Science Standards (draft as of May 2012) were written to include an action verb as a standard. Below are examples of high school biology.

- Obtain and communicate information explaining how the structure and function of systems of specialized cells within organisms help them perform the essential functions of life.

- Communicate information about how DNA sequences determine the structure and function of proteins.

- Develop and use models to explain the hierarchical organization of interacting systems working together to provide specific functions within multicellular organisms

- Analyze and interpret data to identify patterns of behavior that motivate organisms to seek rewards, avoid punishments, develop fears, or form attachments to members of their own species.

What trend do you see for action verbs and assessment? How can you support these assessment goals at home? 
5. Consider the following student scenarios.

- Mike \& Sheila attend the same high school and take many of the same classes.

- Mike is a bright but obstinate student. He consistently gets high grades on classroom quizzes and tests, even though he rarely completes homework assignments and is often tardy. His compositions and reports show keen insight and present thoughtful analyses of critical issues but are usually turned in two or three days late. Because of missing homework and lack of punctuality, Mike receives C's in most of his classes and his grade point average lands him in the middle of his high school ranking. But Mike scores at the highest level on the state tests and qualifies for an honors diploma.

- Shelia is an extremely dedicated and hard-working student. She completes every homework assignment, takes advantage of extra credit options in all of her classes and regularly attends study sessions held by her teachers. Despite her efforts, Shelia often performs poorly on classroom quizzes and tests. Her compositions and reports are well organized and turned in on time and rarely demonstrate more than a surface understanding of critical issues. Shelia also receives Cs in most of her classes and has a class rank similar to Mike. But because she scores at a low level of state tests, Sheila is at risk of receiving an alternative diploma.

a. What are your initial thoughts regarding Mike and Sheila?

b. Does the grade of $\mathrm{C}$ fit either student?

c. Picking between the two students, which scenario would you feel prompted to step in as a parent?

6.

\begin{tabular}{|c|c|c|}
\hline \multicolumn{3}{|c|}{ The Differences Between Summative and Formative Assessment } \\
\hline & Assessment of Learning & Assessment for Learning \\
\hline Assessment form & Summative & Formative \\
\hline Primary users & Teachers, school, district, state & Teachers, students \\
\hline Assess What & $\begin{array}{l}\text { Standards, benchmarks, or } \\
\text { curriculum objectives }\end{array}$ & $\begin{array}{l}\text { Explicit learning targets used in } \\
\text { the course curriculum }\end{array}$ \\
\hline Assess How & Multiple choice or short answer & $\begin{array}{l}\text { Variety of methods best } \\
\text { matching the learning target (i.e. } \\
\text { portfolios, presentations, labs) }\end{array}$ \\
\hline Assess When & $\begin{array}{l}\text { An event after learning, } \\
\text { periodically or annually }\end{array}$ & $\begin{array}{c}\text { Continuous, on-going, a process } \\
\text { during learning }\end{array}$ \\
\hline Typical Uses & $\begin{array}{l}\text { Communicate level of } \\
\text { performance against statistics, } \\
\text { measure achievement at } \\
\text { particular points, aide in } \\
\text { decisions regarding district } \\
\text { resources }\end{array}$ & $\begin{array}{l}\text { Support learning, reflect on } \\
\text { progress made toward objectives, } \\
\text { adjust instruction, provide } \\
\text { descriptive feedback, enable } \\
\text { students to engage in their } \\
\text { learning }\end{array}$ \\
\hline
\end{tabular}


a. Using the chart above, in your opinion, which type of assessment would student motivation more likely be encouraged? Why?

b. Using the chart above, in your opinion, which type of assessment would have more of a positive effect on student learning? Why?

7. ETS along with Pearson \& College Board (2010) produced a summary on the goal of assessment.

"Summative assessment will remain a key element of an educational quality management system, and one of the main goals of this effort is to improve the quality and efficiency of our summative system. However, without questions this goals, we believe that American education would be best served by an integrated system where summative and interim formative components. The structure should provide both accountability and instructionally actionable information" (p. 7).

a. What percentages (of formative \& summative assessments) do you feel as parents provide the most accurate picture of your child's learning? Explain your position.

8. With the emphasis on homework lowered, the assessment for learning strategy encourages more student self-assessment. Andrade and Du (2007) define self-assessment as a portion of formative assessment during "which students reflect on and evaluate the quality of their work and their learning, judge the degree to explicitly stated goals or criteria, and identify strengths and weaknesses in their work" (Andrade and Du, 2007, p. 160).

a. How possible is this task for your student?

b. What supports do students need at home in order to become better assessors of their own learning?

c. What supports do students need at school in order to become better assessors of their own learning?

9. "All students must believe that they can succeed at learning if they try" (Stiggins, 2005, p. 326). Reiterating that an intrinsic drive should always be the motivator to sustain learning, Pink (2009) disarms the carrot and stick mentality to motivate others. Extrinsic motivators typically squash any hope for motivation to come from within the person, it crushes creativity, and it encourages unethical behavior.

a. Therefore, we should focus more on intrinsic motivators. What are the intrinsic motivators that today's teenagers have?

b. What can the school do to support those motivators?

c. What can parents do to support those motivators?

10. What is more valued earning a grade or understanding a course topic?

a. Please explain your choice from the student perspective.

b. Please explain your choice from the parent perspective. 
APPENDIX C

INVITATIONS AND CONSENTS 
Parent Invitation for Informational Night Regarding Final Exams

\section{CENTRAL PRESENTS...}

\section{FRESHMAN FINAL EXAM}

\section{REVIEW NIGHT}

Tuesday, December 4th

$$
\text { 7:00 p.m. }
$$

Lee F. Rosenquist Auditorium

This informational meeting is for both parents and students. Information about the final exam purpose and process will be given along with a review of material from various freshman level courses. Teachers from the English, Reading, Math, Science, and Social Science departments will be there to discuss their final exams. A study packet will be available. A counselor will be there to discuss the final exam schedule. 
Recruitment Invitation to Focus Group

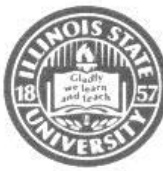

ILLINOIS STATE UNIVERSITY

Illinois' first public university
College of Education

Department of Educational

Administration and Foundations

331 DeGarmo Hall

Campus Box 5900

Normal, IL 61790-5900

Phone: (309) 438-5422

coe.ilstu.edu/eafdept/

Thank you for showing interest at the Freshmen Final Exam Review Night in December in participating in a focus group. As I presented that night, I spoke to you as both a teacher and department chair in Central's Science department and as a doctoral student at Illinois State University. I am currently working on my dissertation. The purpose of my research is to explore parental perception of assessment practices in high school in order to create a stronger partnership between parents and the high school district to support high school students through Assessment for Learning (A4L) changes. Dr. Sharon Michalak of District supports my research endeavors. My advisor during this journey is Dr. Linda Lyman, a professor in the College of Education.

I am requesting your participation, which will involve taking part in a focus group interview session with other parents from the high school district. The focus group will be audiotaped and meet either in Central's Knights of the Round Table room or at West's Warrior Lodge. I expect each focus group session to last approximately 90 minutes. Depending upon interest, an invitation to attend one follow-up session of not more than 90 minutes will be an option. The questions and discussion will revolve around your understanding and opinions of the link between learning and academic performance of high school students, including student self-assessment. At the conclusion of each session, your input regarding the discussion and your comments, questions, and concerns will be asked for in a written survey.

Your participation is voluntary. If you would like to be a part of this research study, please respond to this letter by emailing me at or calling me at so I may call you within 1-2 days to confirm your participation in one the focus group sessions scheduled for February 2013.

Thank you very much for your time and interest in this study. Your opinions will be invaluable to the success of this research study.

Sincerely, Sarah K. Highfill

Telephone Number: 


\section{Informed Consent Form}
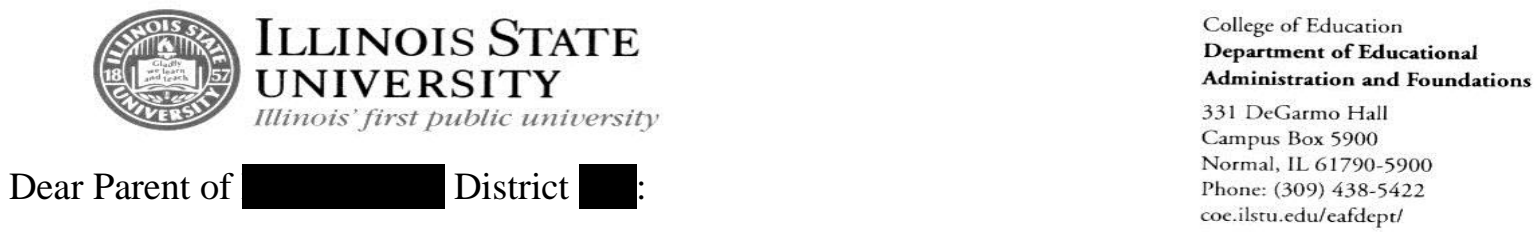

I am a doctoral student under the direction of Dr. Linda Lyman in the College of Education at Illinois State University and supported by Dr. Sharon Michalak, Assistant Superintendent of District $\square$. My dissertation research study is to explore parent perception of assessment practices in high school, and I am requesting your participation in focus group sessions and surveys. I am excited to work within

District where I have been a faculty member since 2000. With my experience as a classroom teacher and department chair for Science at Central, I am interested in creating a partnership with parents as the district continues to address assessment in classroom instruction.

Each focus group session will last 90 minutes in the community rooms of either Central or West High Schools. You may be invited to participate in a second follow-up focus group of no more than 90 minutes if there is interest. At the conclusion of each focus group session, your input of comments, questions, and concerns will be asked for in a written survey. The focus group session will be audio taped with your permission. Questions and discussion will revolve around your understandings of the link between learning and academic performance of high school students, and the role of selfassessment. My hope is that the information gathered will be the first step in establishing collaboration between you and the school district.

Your participation in this study is voluntary. If you choose not to participate or to withdraw from the study at any time, there will be no penalty of any kind. Your decision to participate or not to participate will not affect your partnership with District $\square$ in any way. There are minimal risks to this research study. You may be concerned that the information regarding your perceptions of the high school, revealed in the interview, may be presented to administrators and/or educators of their child's school thus affecting your child's high school experience. Even though this is a natural concern in participating, the research will take a holistic approach to better understanding of assessment practices, and does not have an individual student or parent focus.

Let me assure you that while the results of the research study may be published, your name will not be used. I will take all precautions to maintain your confidentiality (your name will not be used, and the transcript from our interview will not be shared with anyone). Pseudonyms will be used throughout the interview and the writing of the final report. Although there may be no direct benefit to you, the possible benefit of your participation would be to gain the opportunity to become a partner with the district in implementing assessment change that contributes to learning and will benefit your student.

If you have any questions or concerns about the research study, please call me at $\square$, or Dr. Linda Lyman at (309) 438-5238.

Sincerely,

Sarah K. Highfill

I give consent to participate in the above study. I understand that my interview will be audiotaped.

Signature

Date

If you have any questions about your rights as a subject/participant in this research, or if you feel you have been placed at risk, you can contact the Research Ethics \& Compliance Office at Illinois State University at (309) 438-2529. 
APPENDIX D

SELF-ASSESSMENT DOCUMENT 


\section{Example of a Self-Assessment Form}

- My first quarter grade is a $\%$ which is a I am happy/unhappy (circle one) about this grade because:

- On a scale from 1-10, my effort in this class so far has been a because

- Some things I know I did well during first quarter in this class were:

- Some things I know I need to work on for second quarter in this class are:

- My goal in this class for the remainder of the year is:

- I can improve my grade by:

$\bullet$ can help me by:

- My parents/guardians can help me by:

- The thing I have enjoyed most about this class so far is:

- If I could change one thing about this class so far, it would be:

- $\quad$ One thing I really like about Central is:

- $\quad$ One thing I would change about Central would be:

- $\quad$ One more thing I would like I to know about me is:

- If I had one wish right now, it would be:

- Other comments or suggestions I would like to make are: 